\title{
Validation of Cross Sections for Monte Carlo Simulation of the Photoelectric Effect
}

\author{
Min Cheol Han, Han Sung Kim, Maria Grazia Pia, Tullio Basaglia, Matej Batič, Gabriela Hoff, \\ Chan Hyeong Kim, and Paolo Saracco
}

\begin{abstract}
Several total and partial photoionization cross section calculations, based on both theoretical and empirical approaches, are quantitatively evaluated with statistical analyses using a large collection of experimental data retrieved from the literature to identify the state of the art for modeling the photoelectric effect in Monte Carlo particle transport. Some of the examined cross section models are available in general purpose Monte Carlo systems, while others have been implemented and subjected to validation tests for the first time to estimate whether they could improve the accuracy of particle transport codes. The validation process identifies Scofield's 1973 non-relativistic calculations, tabulated in the Evaluated Photon Data Library (EPDL), as the one best reproducing experimental measurements of total cross sections. Specialized total cross section models, some of which derive from more recent calculations, do not provide significant improvements. Scofield's non-relativistic calculations are not surpassed regarding the compatibility with experiment of $K$ and $L$ shell photoionization cross sections either, although in a few test cases Ebel's parameterization produces more accurate results close to absorption edges. Modifications to Biggs and Lighthill's parameterization implemented in Geant4 significantly reduce the accuracy of total cross sections at low energies with respect to its original formulation. The scarcity of suitable experimental data hinders a similar extensive analysis for the simulation of the photoelectron angular distribution, which is limited to a qualitative appraisal.
\end{abstract}

Index Terms-Geant4, Monte Carlo, simulation, x-rays.

\section{INTRODUCTION}

$\mathbf{P}$ HOTOIONIZATION is important in various experimental domains, such as material analysis applications, astrophysics, photon science and bio-medical physics. As one of the interactions photons undergo in matter, it is relevant in experimental methods concerned with the energy deposition resulting from photons as primary or secondary particles. Photoionization is also experimentally relevant for the secondary atomic processes that it induces, X-ray

Manuscript received September 14, 2015; revised January 17, 2016; accepted January 23, 2016. Date of current version April 15, 2016. This work was supported in part by CAPES BEX6460/10-0 and PVE-71/2013 grants, Brazil.

M. C. Han, H. S. Kim, and C. H. Kim are with are with the Department of Nuclear Engineering, Hanyang University, Seoul 133-791, Korea (e-mail: mchan@hanyang.ac.kr; hskim87@hanyang.ac.kr; chkim@hanyang.ac.kr).

M. G. Pia and P. Saracco are with INFN Sezione di Genova, I-16146 Genova, Italy (e-mail: MariaGrazia.Pia@ge.infn.it; Paolo.Saracco@ge.infn.it).

T. Basaglia is with CERN, CH-1211 Geneva 23, Switzerland (e-mail: Tullio.Basaglia@cern.ch).

M. Batič was with INFN Sezione di Genova, Genova, Italy (e-mail: Batic.Matej@gmail.com). He is now with Sinergise, 1000 Ljubljana, Slovenia. G. Hoff is with CAPES, Brasilia, Brazil (e-mail:ghoff.gesic@gmail.com).

Color versions of one or more of the figures in this paper are available online at http://ieeexplore.iee.org.

Digital Object Identifier 10.1109/TNS.2016.2521876 fluorescence and Auger electron emission, which play a role in many physics research areas and technological applications. Extensive reviews, which cover both the theoretical and experimental aspects of this process, can be found in the literature: [1]-[8] are some notable examples among them.

This paper is concerned with modeling the physics of photoionization in a pragmatic way: the simulation of this process in general purpose Monte Carlo codes for particle transport.

Calculations for the simulation of the photoelectric effect are implemented in these codes [9], nevertheless a comprehensive, quantitative appraisal of their validity is not yet documented in the literature. Assessments previously reported in the literature concern comparisons of cross sections with NIST reference values, such as [10], [11], or comparisons of cross section libraries used in Monte Carlo codes [12], or involve complex observables resulting from several physics processes in the full simulation of an experimental set-up, such as [13]. Comparisons with experimental data of basic modeling features of photoionization in Monte Carlo codes, such as those shown in [14], are usually limited to qualitative visual appraisal and to a restricted sample of photon energies and target materials. It is worthwhile to note that the validation of simulation models implies their comparison with experimental measurements [15]; comparisons with tabulations of theoretical calculations or analytical parameterizations, such as those reported in [16] as validation of Geant 4 photon interaction cross sections, do not constitute a validation of the simulation software.

The analysis documented here evaluates the methods adopted in widely known Monte Carlo systems for the calculation of photoelectric cross sections for the elements of the periodic table, as well as other modeling approaches not yet implemented in these codes. This investigation aims to assess the capabilities of Monte Carlo codes for particle transport in this respect, and identify the state of the art for the simulation of the photoelectric effect.

Special emphasis is devoted to the validation and possible improvement of photoionization simulation in Geant4 [17], [18]; nevertheless, the results documented in this paper provide information relevant to other Monte Carlo systems as well.

The simulation of the atomic relaxation following the ionization of an atom has been treated in previous publications [19] — [22], therefore it is not addressed in this paper.

\section{STRATEGY OF THIS STUdY}

This study concerns an extensive set of models for the simulation of photoionization, which are representative of the variety 
of theoretical and empirical methods documented in the literature. The validation test concerns single ionisation of neutral atoms by non-polarized photons, as this is the context handled by general purpose Monte Carlo codes.

Computational performance imposes constraints on the complexity of physics calculations to be performed in the course of simulation; hence the analysis in this paper is limited to theoretical cross sections for which tabulations of pre-calculated values are available and to empirical models that are expressed by means of simple analytical formulations. To be relevant for general purpose Monte Carlo systems, tabulated data should cover the whole periodic table of elements and an extended energy range.

The evaluation mainly concerns total and partial photoelectric cross sections: in particle transport, the former are relevant to determine the occurrence of the photoionization process, while the latter determine which shell is ionized. Calculated cross sections are quantitatively compared with a wide set of experimental data collected from the literature. The compatibility with experiment for each model, and the differences in compatibility with experiment across the various models, are quantified by means of statistical methods.

In addition, methods for the determination of the photoelectron angular distribution are examined; nevertheless, due to the scarcity of pertinent experimental data, their analysis is limited to qualitative considerations.

Computational algorithms pertaining to how basic physics modeling features are used in the transport environment, such as methods for dealing with the macroscopic cross sections for compounds or mixtures [23], [24], are outside the scope of this paper.

\section{Physics OVERVIEW}

Photoionization has been the object of theoretical and experimental interest for several decades; only a brief overview of the physics relevant to the simulation of the photoelectric effect in general purpose Monte Carlo codes is included here to facilitate the understanding of the validation tests reported in this paper.

In the photoelectric effect a photon disappears and an electron is ejected from an atom. The energy of the photoelectron corresponds to the difference between the energy of the absorbed photon and the energy binding the electron to the atom.

General purpose Monte Carlo codes for particle transport consider single photon interactions with isolated atoms in their ground state; they neglect interactions with ions and excited states, and multiple ionizations. Photon interactions are treated regardless of the environment of the target medium; this assumption neglects solid state effects and other features related to the molecular structure of the medium. The environment can have a significant effect on the cross sections near the photoionization thresholds of both inner and outer shell electrons; due to the limitations of their underlying physics assumptions, current general purpose Monte Carlo codes are not usually exploited for the simulation of experimental scenarios involving EXAFS (Extended X-ray Absorption Fine Structure), XANES
(X-ray Absorption Near Edge Structure) and other techniques for which detailed accounting of material structure is required.

\section{A. Total and Partial Cross Sections}

The photoelectric cross section as a function of energy exhibits a characteristic sawtooth behavior corresponding to absorption edges, as the binding energy of each electron subshell is attained and corresponding photoionization is allowed to occur.

Early theoretical calculations of photoionization cross sections were limited to the $\mathrm{K}$ shell; they are represented by the papers of Pratt [25], providing the asymptotic behavior for arbitrarily high energies, and Pratt et al. [26], reporting calculations in the energy range between $200 \mathrm{keV}$ and $2 \mathrm{MeV}$. More extensive calculations became available only at a later stage: Rakavy and Ron [27] calculated cross sections for all subshells of five elements over the energy range $1 \mathrm{keV}$ to $2 \mathrm{MeV}$, and Schmickley and Pratt [28] reported cross sections for $\mathrm{K}$ to $\mathrm{M}$ shells for three elements from 412 to $1332 \mathrm{keV}$.

Scofield's non-relativistic calculations [29] in a HartreeSlater framework represented a major advancement in the field, as they covered systematically all subshells over the whole periodic table of the elements. More recent calculations were performed by Chantler [30], [31] in a self-consistent relativistic Dirac-Hartree-Fock framework.

These theoretical calculations provide the basis for the tabulated data libraries listed in Section IV.

Various empirical formulations of photoionization cross sections are reported in the literature, e.g. in [32]. They derive from fits to experimental data, parameterizations of theoretical calculations and semi-empirical methods involving both measured data and theoretical considerations.

\section{B. Photoelectron Angular Distribution}

Fischer's non-relativistic theory [33] addresses the calculation of differential cross sections in the low energy region. The first relativistic treatment of the photoelectric effect was given by Sauter [34], [35], who calculated the K-shell cross section in the Born approximation; it concerns the lowest order in $\mathrm{Z} \alpha / \beta$ (where $\mathrm{Z}$ is the atomic number of the target, $\alpha$ is the fine structure constant and $\beta$ is $v / c$ ). A comparison of these theories is discussed in [36], which showed that Sauter's theory applies even in the non-relativistic realm, despite being derived for relativistic electrons.

Gavrila [37] and Nagel [38] extended Sauter's results to the next order in $\mathrm{Z} \alpha / \beta$. Further calculations by Gavrila are available for the L shell [39].

Monte Carlo codes generate the photoelectron angular distribution based on these differential cross section calculations; their respective approaches are documented in Section V.

\section{Photoionization Cross Section Compilations}

The photoelectric cross section compilations considered in this study are listed in Table I, which reports the label by which 
TABLE I

COMPILATIONS OF PHOTOIONIZATION CROSS SECTIONS CONSIDERED IN THIS STUDY

\begin{tabular}{lrrrrc}
\hline Identifier & \multicolumn{2}{c}{ Energy range } & Z range & Shell \\
\hline Biggs [50] & $10 \mathrm{eV}$ & $100 \mathrm{GeV}$ & 1 & 100 & no \\
BiggsG4 [50], [53] & $10 \mathrm{eV}$ & $100 \mathrm{GeV}$ & 1 & 100 & no \\
Brennan [60] & $30 \mathrm{eV}$ & $700 \mathrm{keV}$ & 3 & 92 & no \\
Chantler [30], [31] & $10 \mathrm{eV}$ & $433 \mathrm{keV}$ & 1 & 92 & $\mathrm{~K}$ \\
Ebel [71] & $1 \mathrm{keV}$ & $300 \mathrm{keV}$ & 1 & 92 & all \\
Elam [67] & $100 \mathrm{eV}$ & $1 \mathrm{MeV}$ & 1 & 98 & no \\
EPDL97 [62] & $10 \mathrm{eV}$ & $100 \mathrm{GeV}$ & 1 & 100 & all \\
Henke [54] & $10 \mathrm{eV}$ & $30 \mathrm{keV}$ & 1 & 92 & no \\
McMaster [40], [42] & $1 \mathrm{keV}$ & $700 \mathrm{keV}$ & 1 & 94 & no \\
PHOTX [55] & $1 \mathrm{keV}$ & $100 \mathrm{MeV}$ & 1 & 100 & no \\
RTAB [66] & $10 \mathrm{eV}$ & $300 \mathrm{keV}$ & 1 & 99 & all \\
Scofield [29] & $1 \mathrm{keV}$ & $1.5 \mathrm{MeV}$ & 1 & 100 & all \\
StormIsrael [43] & $1 \mathrm{keV}$ & $100 \mathrm{MeV}$ & 1 & 100 & no \\
Veigele [45] & $100 \mathrm{eV}$ & $1 \mathrm{MeV}$ & 1 & 94 & no \\
XCOM-DB [58] & $1 \mathrm{keV}$ & $500 \mathrm{keV}$ & 1 & 100 & no \\
XCOM [58] & $1 \mathrm{keV}$ & $100 \mathrm{GeV}$ & 1 & 100 & no \\
\hline
\end{tabular}

they are identified in the validation analysis, the corresponding references and their coverage in energy, atomic number and atomic shells. A brief overview of their features is summarized in the following subsections, where the compilations appear in chronological order of publication; more details can be found the related references.

\section{A. McMaster's Tables}

The compilation by McMaster et al. [40], [41] reports coherent and incoherent photon scattering cross sections, photoelectric and total cross sections between $1 \mathrm{keV}$ and $1 \mathrm{MeV}$. The data concern elements with atomic numbers 1 to 99 , with the exception of atomic numbers $84,85,87,88,89,91$, and 93 . The photoelectric cross sections were obtained by a combination of methods: by least-squares fitting of semi-empirical data, when they were available, by theoretical calculations from theory in regions where the data were insufficient or were considered unreliable, and by interpolation between semi-empirical and theoretical values, where no data of either kind were available. The semi-empirical photoelectric cross sections were derived by subtracting theoretical scattering cross sections from experimental total photon attenuation data. The theoretical cross sections are based on Schmickley and Pratt's [28] calculations.

The original compilation was updated in 2006 [42].

\section{B. Storm and Israel's Tables}

Storm and Israel's tables [43] encompass photon interaction cross sections for atoms with atomic numbers between 1 and 100 and photon energy from $1 \mathrm{keV}$ to $100 \mathrm{MeV}$. The photoelectric cross sections are derived from several theoretical references: calculations by Brysk and Zerby [44], Rakavy and Ron [27], and Schmickley and Pratt [28].

\section{Veigele's Tables}

The photoelectric cross sections reported in Veigele's compilation [45] are of theoretical origin in the lower energy range (from $100 \mathrm{eV}$ to energies varying between 1 and $10 \mathrm{keV}$, depending on the element) and were calculated with semiempirical methods at higher energies (up to $1 \mathrm{MeV}$ ).

The low energy cross sections derive from non-relativistic, self-consistent field calculations, which are based on independent particle approximation. At higher energies, theoretical scattering cross sections, calculated by relativistic selfconsistent field methods, were subtracted from experimental total attenuation data; the resulting calculated photoelectric cross sections and available photoelectric cross section measurements from $1 \mathrm{keV}$ to $1 \mathrm{MeV}$ were then fitted by a least-squares procedure to produce recommended values.

Veigele's compilation concerns elements with atomic numbers from 1 to 94 .

\section{Scofield's Calculations}

Scofield's 1973 [29] compilation reports photoeffect cross sections for all subshells, for all elements with atomic numbers from 1 to 101 , over the photon energy range between $1 \mathrm{keV}$ and $1.5 \mathrm{MeV}$. The calculations were later extended down to $100 \mathrm{eV} \mathrm{[46].}$

These cross sections derive from non-relativistic calculations based on a solution of the Dirac equation for the orbital electrons moving in a static Hartree-Slater central potential. In this approximation the electron-electron interaction term is replaced with an average value, thus making the calculation less computationally intensive, but in principle also less accurate, than the full Hartree-Fock model, which requires the calculation of the self-consistent field for each term. In the same reference [29] Scofield reports renormalization factors for atomic numbers 2 to 54 to convert the cross sections calculated in the Dirac-Hartree-Slater approximation to values expected from a relativistic Hartree-Fock model.

Comparisons with experimental photon mass attenuation coefficients [46], [47] tend to favour Scofield's unrenormalized values over the renormalized ones. Nevertheless, it is worth noting that these evaluations of Scofield's photoeffect cross sections do not compare the two sets of calculations with direct photoelectric cross sections measurements: the reference data in these comparisons involve the subtraction of theoretically calculated photon scattering contributions from measured photon attenuation coefficients. It is also unclear whether these evaluations rest on a qualitative appraisal only or are based on objective statistical methods.

\section{E. Biggs and Lighthill's Parameterisation}

Biggs and Lighthill [48]-[50] expressed the total photoelectric cross sections as an empirical parameterization of data deriving from various semi-empirical and theoretical sources, which include, among others, Henke's 1982 compilation [51] and the 1978 version of EPDL [52], the latter in turn based on Storm and Israel's, McMaster's, Scofield's and Veigele's compilations.

For element $i$ and energy range $j$ the cross section is represented by the formula:

$$
\sigma_{i j}=\frac{A_{i j 1}}{E}+\frac{A_{i j 2}}{E^{2}}+\frac{A_{i j 3}}{E^{3}}+\frac{A_{i j 4}}{E^{4}}
$$


The tabulations in [50] list the most recent compilation of the $A_{i j}$ coefficients of equation 1 assembled by the original authors of the parameterization.

Modified $A_{i j}$ coefficients for some gases are documented in [53].

\section{F. Henke's Compilation}

The compilation of photoabsorption cross sections by Henke et al. [54] covers the energy range from $10 \mathrm{eV}$ to $30 \mathrm{keV}$, for elements with atomic numbers up to 92 .

Photoabsorption cross sections for energies below $10 \mathrm{keV}$ are based on both theoretical calculations and experimental data, interpolating across the atomic number $\mathrm{Z}$ for elements where experimental data were scarce. Above $10 \mathrm{keV}$ photoabsorption cross sections derive from the semi-empirical parameterizations by Biggs and Lighthill [50].

An earlier compilation by Henke [51], concerning a narrower energy range (from $30 \mathrm{eV}$ to $10 \mathrm{keV}$ ), is based on similar criteria; cross sections from 1.5 to $10 \mathrm{keV}$ are taken from [49].

\section{G. PHOTX and XCOM}

The PHOTX [55], [56] data library was developed as a basis for the photon cross section file of ENDF/B-VI [57]. It provides cross sections for coherent and incoherent scattering, photoelectric absorption, and pair production in the field of the nucleus and in the field of the atomic electrons. The data concern elements with atomic numbers between 1 and 100, and photon energies from $1 \mathrm{keV}$ to $100 \mathrm{MeV}$.

XCOM [58] is a photon cross section database compiled by the NIST (National Institute of Standards and Technology of the United States of America). It concerns elements with atomic numbers up to 100 and photon energies from $1 \mathrm{keV}$ to $100 \mathrm{GeV}$. Two sets of tabulations, identified as XCOM and $\mathrm{XCOM}-\mathrm{DB}$, are evaluated in this paper. XCOM corresponds to the standard energy grid available from the XCOM web site managed by NIST. XCOM-DB is encompassed in the DABAX database, which is part of the X-ray Optics Software Toolkit (XOP) [59]; it is mainly addressed to the photon science experimental community and is limited to photon energies up to $509.5 \mathrm{keV}$.

Regarding the photoelectric effect, both PHOTX and XCOM include Scofield's 1973 unrenormalized cross sections [29] up to $1.5 \mathrm{MeV}$. At higher energies a semi-empirical formula from [32] connects Scofield's values at $1.5 \mathrm{MeV}$ to the asymptotic high energy limit calculated by Pratt [25].

\section{H. Brennan and Cowan's Calculations}

Brennan and Cowan developed a collection of software programs [60] for the calculation of photoabsorption cross sections, atomic scattering factors and other quantities relevant to photon science. The photoelectric cross sections are based on Cromer and Liberman's [61] theory.

Tabulations of photoelectric cross sections derived from Brennan and Cowan's calculations are included in the XOP software system [59].

\section{I. $E P D L$}

The EPDL (Evaluated Photon Data Library) includes photon interaction data concerning photoionization, photoexcitation, coherent and incoherent scattering, and pair and triplet production. The latest version at the time of writing this paper was released in 1997, and is commonly identified as EPDL97 [62]. It is part of the ENDF/B-VII.1 [63] evaluated nuclear data file.

EPDL97 includes total and partial cross sections for elements with atomic numbers between 1 and 100, and for photon energies from $1 \mathrm{eV}$ to $100 \mathrm{GeV}$. Partial cross sections are tabulated for all subshells.

From the edge to $1 \mathrm{MeV}$, subshell ionization cross sections are based on Scofield's data as in [46]; total photoionization cross sections are summed over all subshells. From $1 \mathrm{MeV}$ to $100 \mathrm{GeV}$ the total cross sections are based on Hubbell's data reported in [64]. Scofield's subshell cross sections have been extended up to $100 \mathrm{GeV}$ by ensuring that the sum of the subshell cross sections is equal to HubbeIl's total, and maintaining the same ratio between subshell cross sections over the entire energy range from $1 \mathrm{MeV}$ to $100 \mathrm{GeV}$. At $1 \mathrm{MeV}$ the total photoionization cross section is identical from both sources, therefore the two sets of calculations could be combined in a consistent manner.

EPDL97 documentation reports rough estimates and qualitative comments about the accuracy of the tabulated data, but it does not document how these estimates were produced. To the best of our knowledge systematic, quantitative validation of EPDL97 photoionization data is not documented in the literature.

EPDL97 is extensively used in Monte Carlo simulation; details are given in Section V.

\section{J. Chantler's Calculations}

Chantler calculated photoelectric cross sections in a selfconsistent relativistic Dirac-Hartree-Fock framework [30], [31]. The exchange potential of Chantler's approach follows that of Cromer and Liberman [61], [65] and Brennan and Cowan [60], and is different from the approach used by Scofield [29].

The calculations are carried out in isolated atom and independent particle approximations: each atom is treated as a standalone system, not influenced by other atoms or particles, and each electron is considered to move in an effective potential of the nucleus with the average repulsive force of the electrons. This effective screening neglects some correlation; it also neglects the fact that the potential for one electron is not identical to that of a different electron.

Chantler's published tabulations report total and K-shell cross sections for elements with atomic number from 1 to 92; they cover an energy range comprised between 1-10 eV and $0.4-1 \mathrm{MeV}$ (the lower and upper bounds vary with the atomic number).

\section{K. RTAB}

The RTAB [66] database encompasses three sets of photoionization cross sections along with an extensive set of tabulations 
concerning photon elastic scattering: a set of original calculations (identified in the following as RTAB cross sections), an extension of these cross sections based on EPDL97 (identified as RTABX) and Scofield's 1973 cross sections. They are listed for atomic numbers from 1 to 100 .

All the data in the RTAB database (apart from those reported from other sources) have been consistently computed in the same Dirac-Slater potential. Scofield's 1973 [29] cross sections were also computed in a Dirac-Slater potential, although differently from those included in the RTAB database. According to [66], subshell cross sections are calculated in the Dirac-Slater potential to obtain total atom cross sections from threshold up to several hundred $\mathrm{keV}$. For higher energies, these values are smoothly joined to the total-atom values in the EPDL97, thus extending the cross sections in the RTABX collection up to $100 \mathrm{GeV}$. RTAB photoelectric cross sections are tabulated for energies up to $300 \mathrm{keV}$.

The RTAB photoelectric database has not been exploited yet in general purpose Monte Carlo systems.

\section{Elam's Database}

Elam's database [67] provides photon cross section data for elements with atomic numbers between 1 and 98, and for photon energy from $100 \mathrm{eV}$ to $1 \mathrm{MeV}$.

Above $1 \mathrm{keV}$ the photoabsorption cross sections derive from the XCOM ones of [68], which were parameterized with a cubic spline algorithm. Below $1 \mathrm{keV}$ they are based on the 1981 version of EPDL [69]. Appropriate algorithms were applied to the data to ensure a smooth connection between the two sets of cross sections. Edge discontinuities were adjusted to be consistent with Williams' atomic binding energies [70].

\section{Ebel's Parameterizations}

Ebel et al. [71] developed parameterizations of total photoelectric absorption coefficients and of subshell absorption coefficients in the energy range from $1 \mathrm{keV}$ to $300 \mathrm{keV}$, for elements with atomic number up to 92 . They are based on fitting fifth order polynomials in the logarithm of the photon energy to Scofield's 1973 [29] cross section data. The coefficients for the parameterization are tabulated for each energy interval identified by the absorption edges of a given element. Photoelectric cross sections can be calculated using the tabulations.

This compilation was developed especially for application in fundamental parameter programs for quantitative $\mathrm{X}$-ray analysis.

\section{Photolonization in Monte Carlo Codes}

General purpose Monte Carlo codes for particle transport include algorithms for the simulation of the photoelectric effect.

The original version of EGS4 [72] calculated photoelectric total cross sections based on Storm and Israel's tables [43] and generated the photoelectron with the same direction as the incident photon. Later evolutions introduced the use of PHOTX cross sections [73] and the generation of the photoelectron angular distribution [74] based on Sauter's theory [34]. These features are currently implemented in EGS5 [14]. EGSnrc [75] provides the option of calculating total photoelectric cross sections based on Storm and Israel's tables as originally in EGS4 or on a fit to XCOM [58] cross sections, while it uses subshell cross sections based on EPDL [62]. It samples the photoelectron angular distribution according to the method described in [74] based on Sauter's theory.

ETRAN [76] uses Scofield's 1973 [29] cross sections for energies from $1 \mathrm{keV}$ to $1.5 \mathrm{MeV}$ and extends them to higher energies by exploiting Hubbell's method [32] to connect the values at $1.5 \mathrm{MeV}$ to the asymptotic high energy limit calculated by Pratt [25]. It samples the direction of the photoelectron from Fischer's [33] distribution for electron energies below $50 \mathrm{keV}$ and from Sauter's [34] distribution for higher energies.

FLUKA [77], [78] calculates photoelectric cross sections based on EPDL97 and samples the photoelectron direction according to Sauter's theory [34].

ITS [79] calculates photoelectric cross sections based on Scofield's 1973 non-renormalized values. The angle of the photoelectron with respect to the parent photon is described by Fischer's distribution [33] at lower energies and by Sauter's [34] formula at higher energies.

MCNP5 [80] and MCNPX [81] provided different options of data libraries for the calculation of photoelectric cross sections: two version of EPDL (EPDL97 [62] and EPDL89 [82]), and ENDF/B-IV [84] data complemented by Storm and Israel's tables [43] for atomic numbers greater than 83. MCNP6 [83] has extended the minimum energy cut-off for photon transport down to $1 \mathrm{eV}$; the necessary photo-atomic cross sections derive from ENDF/B-VI version 8, which in turn is based on EPDL97 regarding photon interactions.

In the first version of Penelope including photon transport [85] photoelectric cross sections were interpolated from XCOM; in more recent versions [86], [87] they are interpolated from EPDL97 tabulations. The photoelectron angular distribution is sampled from Sauter's differential cross section for the K shell [34].

GEANT 3 [88] calculated total photoionization cross sections based on Biggs and Lighthill's [50] parameterizations; the probability of ionization of the $\mathrm{K}$ shell and $\mathrm{L}$ subshells was estimated by parameterizations of the jump ratios deriving from Veigele's [45] tables. The angular distribution of the photoelectron was sampled for the $\mathrm{K}$ shell and for the $\mathrm{L}_{1}, \mathrm{~L}_{2}$ and $\mathrm{L}_{3}$ subshells based on Sauter's [34], [35] and Gavrila's [37], [39] calculations.

The Geant 4 toolkit encompasses various implementations of the photoelectric effect. The overview summarized here concerns the latest version at the time of writing this paper: Geant4 10.1 , complemented by two correction patches.

The model implemented in Geant 4 standard electromagnetic package calculates cross sections based on the analytical formula of Biggs and Lighthill, but it uses modified coefficients deriving from a fit to experimental data. The related reference cited in Geant4 Physics Reference Manual [89] as the source of these modifications does not appear to be consistent, presumably due to a mismatch between the Russian and English versions of the periodical where it was published. The modifications appear to derive from [53], which reports fits 
to experimental data concerning noble gases, hydrogen, carbon, fluorine, oxygen and silicon; they concern cross sections at low energies. The atomic cross section calculation according to Biggs-Lighthill parameterization is implemented in the G4SandiaTable and G4StaticSandiaData classes of Geant4 materials package. The same calculation of photoelectric cross sections is also used by the PAI (PhotoAbsorption-Ionisation) model [90]. The energy of the emitted photoelectron is determined as the difference between the energy of the interacting photon and the binding energy of the ionized shell defined in the G4AtomicShells class [22], and the photoelectron angle is calculated according to the Sauter-Gavrila distribution for $\mathrm{K}$ shell [34], [37].

Geant4 low energy electromagnetic package [91], [92] encompasses two implementations of the photoelectric effect, one identified as "Livermore" [93] and one reengineered from the 2008 version of the Penelope code [86]; both models calculate total and partial cross sections based on EPDL97. The so-called "Livermore" model provides three options of computing the angular distribution of the emitted photoelectron: in the same direction as the incident photon, based on Gavrila's distribution of the polar angle [37] for the $\mathrm{K}$ shell and the $\mathrm{L}_{1}$ subshell, and based on a double differential cross section derived from Gavrila's [37], [39] calculations, which can also handle polarized photons.

In addition, the Geant 4 toolkit encompasses two models for the simulation of the photoelectric effect concerning polarized photons: one for circularly polarised photons in the polarisation package and one in the low energy electromagnetic package, identified as "Livermore polarized". Their evaluation is outside the scope of this paper.

\section{The Validation Process}

The validation process adopts the same methodology used in [94] for the validation of photon elastic scattering. The main concepts and most relevant features of the validation method are summarized below to facilitate understanding of the results reported in Sections VII and VIII.

\section{A. Simulation Models}

The validation process concerns the methods for the calculation of total and subshell cross sections summarized in Table I. Some of these simulation models represent novel approaches with respect to those so far available in Geant4 and in other general purpose Monte Carlo codes.

All the physics models subject to evaluation have been implemented in a consistent software design, compatible with the Geant4 toolkit, which minimizes external dependencies to ensure unbiased appraisal of their intrinsic behaviour. The software adopts a policy-based class design [95], which supports the provision of a wide variety of physics models without imposing the burden of inheritance from a predefined interface.

A single policy class calculates cross sections that exploit tabulations; alternative tabulations, corresponding to different physics models, are managed through the file system. Dedicated policy classes implement cross section calculations based on analytical formulae. The same scheme is adopted for total and partial cross section calculation; the latter is involved in the algorithm that creates a vacancy, which drives the subsequent atomic relaxation process. Alternative modeling of photoelectron angular distributions is handled through a Strategy pattern [100].

A photoionization process, derived from the G4VDiscreteProcess class of Geant4 kernel, which in turn is derived from G4VProcess, acts as a host class for the policy classes; they can be interchanged [96]-[98] to determine its behaviour. The simulation of photoionization according to this software design is consistent with Geant4 kernel, since Geant4 tracking handles all processes polymorphically through the G4VProcess base class.

Since policy classes are characterized by a single responsibility and have minimal dependencies on other parts of the software, the adopted programming paradigm enables independent modeling and test of all physics options. Their validation can be performed through simple unit tests. This strategy ensures greater modeling flexibility and testing agility than the one adopted in the Geant4 electromagnetic package, where total cross section, vacancy creation and final state generation are bundled into one object: that software design choice requires full-scale simulation applications to test basic physics entities, due to extensive dependencies imposed by the G4VEmModel base class, from which all photoelectric models derive.

Existing physics models in Geant4 have been refactored [101] consistently with this software design; other models not yet available in Geant 4 have been implemented for the first time. The correctness of the implementation has been verified prior to the validation process to ensure that the software reproduces the physical features of each model consistently.

\section{B. Experimental Data}

Experimental data [102]-[181] for the validation of the simulation models were collected from a survey of the literature. The sample of experimental total cross sections consists of approximately 3000 measurements, which concern 61 target atoms and span energies approximately from $5 \mathrm{eV}$ to $1.2 \mathrm{MeV}$. It includes measurements at energies below $100 \mathrm{eV}$, mostly concerning gaseous targets: these data are relevant to evaluate the accuracy of calculations performed in independent particle approximation at very low energies, e.g. the EPDL97 data library, which extend down to $1 \mathrm{eV}$.

The sample of subshell cross sections encompasses approximately 600 measurements, which concern 52 target atoms and span energies approximately from $1 \mathrm{eV}$ to $2.75 \mathrm{MeV}$.

An overview of the experimental data sample is summarized in Tables II-III.

The photoionization cross sections reported in the literature as experimentally measured often derive from measurements of total photon attenuation, from which theoretically calculated contributions from photon scattering were subtracted. These semi-empirical values are not appropriate to an epistemologically correct validation process, which requires the comparison of simulation models with truly experimental data. An evaluation of the possible systematic effects induced by using 
TABLE II

SUMMARY OF THE EXPERIMENTAL TOTAL CROSS SECTION DATA USED IN THE VALIDATION ANALYSIS

\begin{tabular}{|c|c|c|c|c|}
\hline Atomic Number & Element & Energy range $(\mathrm{keV})$ & Sample size & References \\
\hline 1 & $\mathrm{H}$ & $0.0136-0.020$ & 27 & {$[102]-[104]$} \\
\hline 2 & $\mathrm{He}$ & $0.025-0.277$ & 320 & {$[105]-[112]$} \\
\hline 3 & $\mathrm{Li}$ & $0.046-0.400$ & 93 & [113]-[116] \\
\hline 7 & $\mathrm{~N}$ & $0.015-0.4$ & 73 & [117], [118] \\
\hline 8 & $\mathrm{O}$ & $0.013-0.28$ & 215 & {$[103],[119]-[121]$} \\
\hline 10 & $\mathrm{Ne}$ & $0.022-2.952$ & 448 & {$[108],[110]-[112],[117],[122]$} \\
\hline 11 & $\mathrm{Na}$ & $0.046-0.246$ & 17 & [123] \\
\hline 13 & $\mathrm{Al}$ & 145.4 & 1 & [124] \\
\hline 17 & $\mathrm{Cl}$ & $0.016-0.078$ & 25 & {$[125]$} \\
\hline 18 & $\mathrm{Ar}$ & $0.016-6$ & 487 & {$[105],[110]-[112],[117],[126]-[130]$} \\
\hline 19 & $\mathrm{~K}$ & $0.004-0.005$ & 12 & [131] \\
\hline 22 & $\mathrm{Ti}$ & 59.54 & 1 & {$[132]$} \\
\hline 23 & V & 59.54 & 1 & [132] \\
\hline 24 & $\mathrm{Cr}$ & 59.54 & 1 & {$[132]$} \\
\hline 25 & $\mathrm{Mn}$ & 59.54 & 1 & {$[132]$} \\
\hline 26 & $\mathrm{Fe}$ & $0.008-59.54$ & 25 & {$[132]-[134]$} \\
\hline 27 & Co & 59.54 & 1 & {$[132]$} \\
\hline 28 & $\mathrm{Ni}$ & $1.487-59.54$ & 17 & {$[132],[133]$} \\
\hline 29 & $\mathrm{Cu}$ & $59.54-661.6$ & 9 & {$[124],[132],[135]-[138]$} \\
\hline 30 & $\mathrm{Zn}$ & 59.54 & 1 & [132] \\
\hline 33 & As & 59.54 & 1 & [132] \\
\hline 34 & $\mathrm{Se}$ & 59.54 & 1 & {$[132]$} \\
\hline 36 & $\mathrm{Kr}$ & $0.015-1.626$ & 357 & {$[105],[110],[112],[139],[140]$} \\
\hline 37 & $\mathrm{Rb}$ & $0.004-0.010$ & 4 & [141] \\
\hline 38 & $\mathrm{Sr}$ & 59.54 & 1 & {$[132]$} \\
\hline 39 & Y & $279.2-661.6$ & 2 & [142] \\
\hline 40 & $\mathrm{Zr}$ & $59.54-661.6$ & 7 & {$[124],[135]-[137],[143]$} \\
\hline 41 & $\mathrm{Nb}$ & 59.54 & 2 & [143] \\
\hline 42 & Mo & $59.54-661.6$ & 7 & {$[132],[138],[143]$} \\
\hline 43 & $\mathrm{Tc}$ & 59.54 & 1 & {$[143]$} \\
\hline 44 & $\mathrm{Ru}$ & 59.54 & 1 & [143] \\
\hline 45 & $\mathrm{Rh}$ & 59.54 & 1 & [143] \\
\hline 46 & $\mathrm{Pd}$ & 59.54 & 1 & {$[143]$} \\
\hline 47 & $\mathrm{Ag}$ & $1.487-661.6$ & 13 & {$[124],[?],[135]-[137],[143]-[146]$} \\
\hline 48 & $\mathrm{Cd}$ & 59.54 & 2 & [132], [143] \\
\hline 49 & In & 59.54 & 2 & [143] \\
\hline 50 & Sn & $1-661.6$ & 31 & {$[124],[133],[135]-[137],[143],[144],[146]$} \\
\hline 51 & $\mathrm{Sb}$ & 59.54 & 1 & {$[143]$} \\
\hline 52 & $\mathrm{Te}$ & 59.54 & 2 & [143] \\
\hline 54 & $\mathrm{Xe}$ & $0.013-6$ & 657 & [110], [122], [128], [130], [147] \\
\hline 55 & Cs & $0.004-59.54$ & 14 & {$[132],[141],[148]$} \\
\hline 57 & $\mathrm{La}$ & 59.54 & 1 & [149] \\
\hline 58 & $\mathrm{Ce}$ & $59.54-661.6$ & 6 & {$[142],[149]-[152]$} \\
\hline 59 & $\operatorname{Pr}$ & $59.54-661.6$ & 3 & {$[150]-[152]$} \\
\hline 60 & Nd & $59.54-661.6$ & 2 & {$[150],[152]$} \\
\hline 61 & $\mathrm{Pm}$ & 59.54 & 1 & {$[152]$} \\
\hline 62 & $\mathrm{Sm}$ & $59.54-145.4$ & 4 & [149], [151], [152] \\
\hline 63 & $\mathrm{Eu}$ & 59.54 & 2 & {$[149],[152]$} \\
\hline 64 & Gd & $59.54-661.6$ & 5 & {$[150]-[153]$} \\
\hline 65 & $\mathrm{~Tb}$ & 59.54 & 3 & [149], [152], [153] \\
\hline 66 & Dy & $59.54-661.6$ & 8 & {$[142],[149]-[153]$} \\
\hline 67 & Но & 59.54 & 1 & {$[152]$} \\
\hline 68 & $\mathrm{Er}$ & 59.54 & 3 & [149], [152], [153] \\
\hline 70 & $\mathrm{Yb}$ & $279.2-661.6$ & 2 & [142] \\
\hline 72 & Hf & $65.839-68.547$ & 25 & [154] \\
\hline 73 & $\mathrm{Ta}$ & $1.487-661.6$ & 31 & {$[124],[133],[135]-[138],[144]$} \\
\hline 78 & $\mathrm{Pt}$ & $1-40$ & 25 & {$[133]$} \\
\hline 79 & $\mathrm{Au}$ & $0.93-661.6$ & 33 & [133], [135]-[137], [145] \\
\hline 80 & $\mathrm{Hg}$ & 1173 & 1 & [155] \\
\hline 82 & $\mathrm{~Pb}$ & $1.487-1173$ & 36 & {$[124],[133],[135]-[137],[144]-[146],[155],[156]$} \\
\hline 92 & $\mathrm{U}$ & $1.487-3$ & 4 & {$[133]$} \\
\hline
\end{tabular}

semi-empirical data [182]-[245] as a reference for testing cross section calculations is reported in Section VII-A; it concerns a sample of approximately 1500 total cross sections, spanning energies between approximately $50 \mathrm{eV}$ and $6 \mathrm{MeV}$.

Some experimental measurements have been published only in graphical form; numerical values were extracted from the plots by means of the PlotDigitizer [246] digitizing software. The error introduced by the digitization process was estimated by digitizing a few experimental data samples, which are reported in the related publications both in graphical and numerical format. The reliability of the digitized values is hindered by the difficulty of appraising the experimental points 
TABLE III

SUMMARY OF THE EXPERIMENTAL SUBSHELL CROSS SECTION DATA USED IN THE VALIDATION ANALYSIS

\begin{tabular}{|c|c|c|c|c|c|}
\hline $\mathrm{Z}$ & Element & Shell & Energy range (keV) & Sample size & References \\
\hline 3 & $\mathrm{Li}$ & $\mathrm{K}$ & $0.069-1.487$ & 10 & [115] \\
\hline 10 & $\mathrm{Ne}$ & $\mathrm{L}_{1}$ & $0.006-0.127$ & 14 & [158] \\
\hline 18 & $\mathrm{Ar}$ & $\mathrm{M}_{1}$ & $0.001-0.1$ & 147 & {$[159]-[162]$} \\
\hline 20 & $\mathrm{Ca}$ & $\mathrm{M}_{1}, \mathrm{~N}_{1}$ & $0.038-0.121$ & 24 & {$[157]$} \\
\hline 25 & $\mathrm{Mn}$ & $\mathrm{M}_{1}$ & $0.113-0.27$ & 7 & [163] \\
\hline 26 & $\mathrm{Fe}$ & $\mathrm{K}$ & 59.54 & 1 & [164] \\
\hline 28 & $\mathrm{Ni}$ & $\mathrm{K}$ & 59.54 & 1 & [164] \\
\hline 29 & $\mathrm{Cu}$ & $\mathrm{K}$ & $59.54-441.8$ & 3 & [164], [165] \\
\hline 30 & $\mathrm{Zn}$ & $\mathrm{K}$ & 59.54 & 1 & [164] \\
\hline 33 & As & $\mathrm{K}$ & 59.54 & 1 & [164] \\
\hline 34 & $\mathrm{Se}$ & $\mathrm{K}$ & 59.54 & 1 & {$[164]$} \\
\hline 35 & $\mathrm{Br}$ & $\mathrm{K}$ & 59.54 & 1 & [164] \\
\hline 36 & $\mathrm{Kr}$ & $\mathrm{N}_{1}$ & $0.028-0.041$ & 5 & {$[162]$} \\
\hline 40 & $\mathrm{Zr}$ & $\mathrm{K}, \mathrm{L}_{1}-\mathrm{L}_{3}$ & $59.54-441.8$ & 15 & {$[124],[164]-[166]$} \\
\hline 41 & $\mathrm{Nb}$ & $\mathrm{K}, \mathrm{L}, \mathrm{L}_{1}-\mathrm{L}_{3}$ & 59.54 & 6 & [164], [166] \\
\hline 42 & Mo & $\mathrm{K}, \mathrm{L}, \mathrm{L}_{1}-\mathrm{L}_{3}$ & $5.96-74.4$ & 6 & [164], [166] \\
\hline 43 & $\mathrm{Tc}$ & $\mathrm{K}, \mathrm{L}_{1}-\mathrm{L}_{3}$ & 59.54 & 4 & {$[166]$} \\
\hline 44 & $\mathrm{Ru}$ & $\mathrm{K}, \mathrm{L}_{1}-\mathrm{L}_{3}$ & 59.54 & 4 & [166] \\
\hline 45 & $\mathrm{Rh}$ & $\mathrm{K}, \mathrm{L}_{1}-\mathrm{L}_{3}$ & 59.54 & 4 & [166] \\
\hline 46 & $\mathrm{Pd}$ & $\mathrm{K}, \mathrm{L}_{1}-\mathrm{L}_{3}$ & 59.54 & 4 & [166] \\
\hline 47 & $\mathrm{Ag}$ & $\mathrm{K}, \mathrm{L}, \mathrm{L}_{1}-\mathrm{L}_{3}$ & $59.54-441.8$ & 8 & {$[124],[164]-[166]$} \\
\hline 48 & $\mathrm{Cd}$ & $\mathrm{K}, \mathrm{L}, \mathrm{L}_{1}-\mathrm{L}_{3}$ & 59.54 & 4 & [166] \\
\hline 49 & In & $\mathrm{K}, \mathrm{L}, \mathrm{L}_{1}-\mathrm{L}_{3}$ & 59.54 & 6 & [164], [166] \\
\hline 50 & Sn & $\mathrm{K}, \mathrm{L}, \mathrm{L}_{1}-\mathrm{L}_{3}$ & $59.54-1330$ & 19 & {$[124],[164]-[166],[167],[168],[169]$} \\
\hline 51 & $\mathrm{Sb}$ & $\mathrm{K}, \mathrm{L}_{1}-\mathrm{L}_{3}$ & 59.54 & 4 & [166] \\
\hline 52 & $\mathrm{Te}$ & $\mathrm{K}, \mathrm{L}_{1}-\mathrm{L}_{3}$ & 59.54 & 4 & [166] \\
\hline 53 & I & $\mathrm{K}$ & 59.54 & 2 & [164] \\
\hline 54 & $\mathrm{Xe}$ & $\mathrm{M}_{4}, \mathrm{M}_{5}, \mathrm{O}_{1}$ & $0.0236-1$ & 66 & {$[162],[170],[171]$} \\
\hline 56 & $\mathrm{Ba}$ & $\mathrm{O}_{1}$ & $0.069-0.119$ & 28 & [172] \\
\hline 58 & $\mathrm{Ce}$ & $\mathrm{K}, \mathrm{L}, \mathrm{L}_{1}-\mathrm{L}_{3}$ & 59.54 & 4 & [166] \\
\hline 59 & $\operatorname{Pr}$ & $\mathrm{K}, \mathrm{L}_{1}-\mathrm{L}_{3}$ & 59.54 & 4 & [166] \\
\hline 60 & $\mathrm{Nd}$ & $\mathrm{K}, \mathrm{L}_{1}-\mathrm{L}_{3}$ & 59.54 & 4 & [166] \\
\hline 61 & $\mathrm{Pm}$ & $\mathrm{K}, \mathrm{L}_{1}-\mathrm{L}_{3}$ & 59.54 & 4 & [166] \\
\hline 62 & $\mathrm{Sm}$ & $\mathrm{K}, \mathrm{L}, \mathrm{L}_{1}-\mathrm{L}_{3}$ & 59.54 & 4 & [166] \\
\hline 63 & $\mathrm{Eu}$ & $\mathrm{K}, \mathrm{L}, \mathrm{L}_{1}-\mathrm{L}_{3}$ & 59.54 & 4 & [166] \\
\hline 64 & Gd & $\mathrm{K}, \mathrm{L}_{1}-\mathrm{L}_{3}$ & $50.3-59.54$ & 5 & [166], [173] \\
\hline 65 & $\mathrm{~Tb}$ & $\mathrm{~K}, \mathrm{~L}, \mathrm{~L}_{1}-\mathrm{L}_{3}$ & 59.54 & 4 & [166] \\
\hline 66 & Dy & $\mathrm{K}, \mathrm{L}, \mathrm{L}_{1}-\mathrm{L}_{3}$ & $53.8-59.54$ & 5 & {$[166],[174]$} \\
\hline 67 & Ho & $\mathrm{K}, \mathrm{L}_{1}-\mathrm{L}_{3}$ & 59.54 & 4 & [166] \\
\hline 68 & Er & $\mathrm{K}, \mathrm{L}, \mathrm{L}_{1}-\mathrm{L}_{3}$ & 59.54 & 4 & [166] \\
\hline 70 & $\mathrm{Yb}$ & $\mathrm{K}$ & 61.34 & 1 & [174] \\
\hline 72 & Hf & $\mathrm{K}$ & $65.29-65.44$ & 2 & [173], [175] \\
\hline 73 & $\mathrm{Ta}$ & $\mathrm{K}$ & $67.36-1330$ & 15 & [124], [165], [167]-[169], [173], [175] \\
\hline 74 & W & $\mathrm{K}$ & $59.57-69.64$ & 2 & {$[174],[176]$} \\
\hline 78 & $\mathrm{Pt}$ & $\mathrm{K}$ & 1330 & 1 & [155] \\
\hline 79 & $\mathrm{Au}$ & $\mathrm{K}, \mathrm{N}_{6}-\mathrm{N}_{7}, \mathrm{O}_{1}-\mathrm{O}_{3}, \mathrm{P}_{1}$ & $5-1330$ & 60 & [26], [165], [167], [169], [173], [175], [177] \\
\hline 80 & $\mathrm{Hg}$ & $\mathrm{K}, \mathrm{L}$ & $59.54-1250$ & 2 & [155], [176] \\
\hline 81 & $\mathrm{Tl}$ & $\mathrm{L}$ & $59.54-74.4$ & 3 & {$[176],[178]$} \\
\hline 82 & $\mathrm{~Pb}$ & $\mathrm{~K}, \mathrm{~L}, \mathrm{~L}_{3}$ & $13.6-2750$ & 28 & {$[26],[124],[155],[165],[167]-[169],[173],[175],[176],[178]-[180]$} \\
\hline 83 & $\mathrm{Bi}$ & $\mathrm{K}, \mathrm{L}$ & $59.54-1330$ & 4 & [155], [176], [178] \\
\hline 90 & Th & $\mathrm{K}, \mathrm{L}_{3}$ & $16.9-1330$ & 9 & {$[167],[169],[179]$} \\
\hline 92 & $\mathrm{U}$ & $\mathrm{K}, \mathrm{L}, \mathrm{L}_{3}$ & $17.8-1330$ & 10 & {$[155],[176],[178],[179],[181]$} \\
\hline
\end{tabular}

and their error bars in figures that may span several orders of magnitude in logarithmic scale, or that appear of questionable graphical quality in the original publication. Caution was exercised in using these digitized data in the validation analysis; they were discarded, if incompatible with other measurements reported in the literature in numerical form.

Large discrepancies are evident in some of the experimental data; systematic effects are probably present in some cases, where sequences of positive or negative differences between data samples originating from different experimental groups are qualitiatively visible, and confirmed by the WaldWolfowitz test [247] to be incompatible with randomness. Experimental data exhibiting large discrepancies with respect to other measurements in similar configurations, which hint at the presence of systematic effects, are excluded from the validation tests.

The validation process is hindered by physical effects related to the structure of the target material. Accuracy of edge position is limited by chemical shifts and the detailed structure of the experimental material observed. Usually an accuracy of absolute energies below $1-3 \mathrm{eV}$ is unattainable for this reason. At low energies (less than 200-500 eV) the occurrence of collective valence effects and dipole resonances can lead to much larger deviations (e.g. up to $50 \mathrm{eV}$ or $10 \%$ ). General purpose Monte Carlo codes do not take into account such material structure effects; the cross sections they use for the simulation of 
the photoionization process, briefly outlined in Section IV, are not intended to model these features. This limitation should be taken into account in the evaluation of the results of the validation process.

Correct estimate of experimental errors is a concern in the validation of physics models, since unrealistic estimation of the experimental errors may lead to incorrect conclusions regarding the rejection of the null hypothesis in tests whose statistic takes experimental uncertainties explicitly into account. Although technological developments have contributed to improved precision of measurement, some estimates of experimental uncertainties reported in the literature may be excessively optimistic, especially when they appear inconsistent with other measurements exploiting similar experimental techniques. Experimental measurements claiming much smaller uncertainties than similar ones have been critically evaluated in the analysis process.

\section{Data Analysis}

The evaluation of the simulation models performed in this study has two objectives: to validate them quantitatively, and to compare their relative capabilities.

The scope of the software validation process is defined according to the guidelines of the IEEE Standard devoted to software verification and validation [248], which conforms to the life cycle process standard defined in ISO/IEC Standard 12207 [249]. For the problem domain considered in this paper, the validation process provides evidence that the software models photoionization consistently with experiment.

A quantitative analysis, based on statistical methods, is practically possible only for the validation of cross sections, for which a large sample of experimental data is available. The scarcity of angular distribution data in the literature hinders the validation of simulation models through similar statistical analysis methods: only qualitative general considerations can be made.

The statistical analysis of photoionization cross sections is articulated over two stages: the first determines the compatibility between the cross sections calculated by each simulation model and experimental data, while the second determines whether the various models exhibit any significant difference in compatibility with experiment. The Statistical Toolkit [250], [251] and R [252] are used in the statistical analysis. The level of significance of the tests is 0.01 , unless stated otherwise.

The first stage of the analysis encompasses a number of test cases, each one corresponding to a configuration (characterized by photon energy, target element, experimental source and, if appropriate, subshell) for which experimental data are available. The inclusion of the experimental source in the definition of a test case facilitates the identification of possible systematic effects related to the experimental environment of the measurements. For each test case, cross sections calculated by the software are compared with measured ones by means of goodness-of-fit tests. The null hypothesis is defined as the equivalence of the simulated and experimental data distributions subject to comparison, as being drawn from the same parent distribution. The goodness-of-fit analysis is primarily based on the $\chi^{2}$ test [253]. Among goodness-of-fit tests, this test has the property of taking experimental uncertainties explicitly into account; consequently, the test statistic is sensitive to their correct appraisal.

The "efficiency" of a physics model is defined as the fraction of test cases in which the $\chi^{2}$ test does not reject the null hypothesis. This variable quantifies the capability of that simulation model to produce results statistically consistent with experiment over the whole set of test cases, which in physical terms means over the whole range of photon energies and target elements involved in the validation process. Two methods were applied to calculate the uncertainties on the efficiencies: the conventional method involving the binomial distribution, described in many introductory statistics textbooks (e.g. [254]), and a method based on Bayes' theorem [255]. The two methods deliver identical results within the number of significant digits reported in the following tables; the method based on Bayes' theorem delivers meaningful results, which are reported in the following sections, also in limiting cases, i.e. for efficiencies very close to 0 or to 1 , where the conventional method based on the binomial distribution produces unreasonable values.

The second stage of the statistical analysis quantifies the differences of the simulation models in compatibility with experiment. It consists of a categorical analysis based on contingency tables, which derive from the results of the $\chi^{2}$ test: the outcome of this test is classified as "fail" or "pass", according to whether the null hypothesis is rejected or not, respectively. The simulation model exhibiting the largest efficiency is considered as a reference in the categorical analysis; the other models are compared to it, to determine whether their difference in compatibility with measurements is statistically significant.

The null hypothesis in the analysis of a contingency table assumes equivalent compatibility with experiment of the cross section models it compares.

A variety of tests is applied to determine the statistical significance of the difference between the data categories (i.e. cross section models) subject to evaluation: Pearson's $\chi^{2}$ test [256] (when the number of entries in each cell of the table is greater than 5), Fisher's exact test [257], Boschloo's [258] test, the test based on Suissa and Schuster's Z-pooled statistic [259], Santner and Snell's test [260] and Barnard's test [261]. As some contingency tables contain cells with a large number of entries ( $>100)$, Barnard's test was calculated according to the approximate CSM statistic [262] to reduce the computational burden. The use of a variety of tests mitigates the risk of introducing systematic effects in the validation results due to peculiarities in the mathematical formulation of the test statistic.

Fisher's test is widely used in the analysis of contingency tables. It is based on a model in which both the row and column sums are fixed in advance, which seldom occurs in experimental practice; it remains applicable to cases in which the row or column totals, or both, are not fixed, but in these cases it tends to be conservative, yielding a larger $\mathrm{p}$-value than the true significance of the test [264].

Unconditional tests, such as Barnard's test [261], Boschloo's test [258] and Suissa and Shuster's [259] calculation of a Z-pooled statistic, are deemed more powerful than Fisher's 


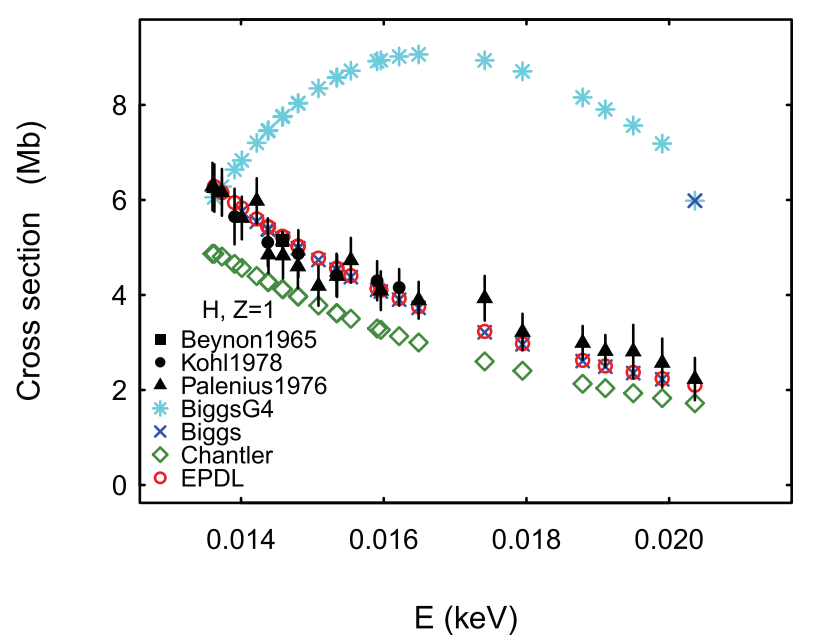

Fig. 1. Total photoionization cross section for $\mathrm{Z}=1$ as a function of photon energy.

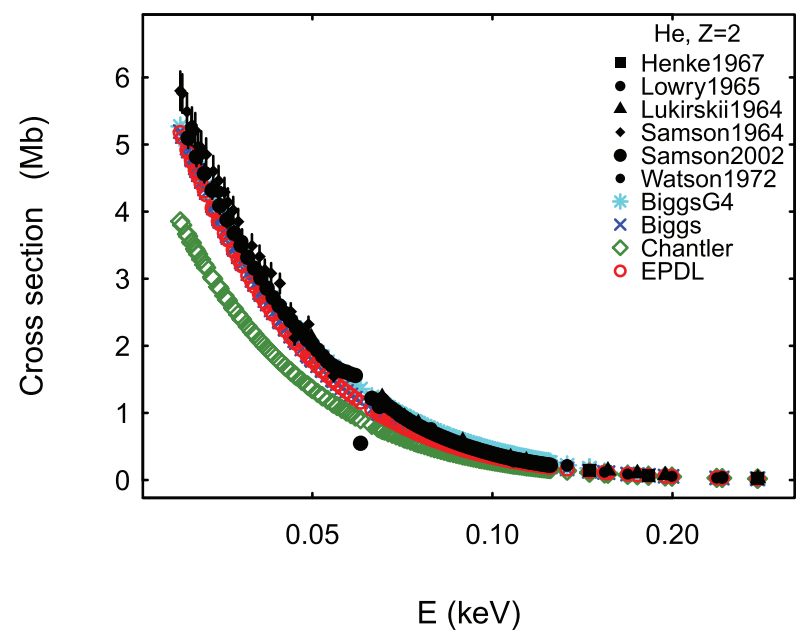

Fig. 2. Total photoionization cross section for $\mathrm{Z}=2$ as a function of photon energy.

exact test in some configurations of $2 \times 2$ contingency tables [265], [266], but they are computationally more intensive.

\section{Results of Total CROSS SECtion VAlidATION}

Figs. 1 to 24 illustrate calculated and experimental total cross sections.

The validation analysis encompasses various areas of investigation: the evaluation of possible systematic effects related to the characteristics of reference data, the evaluation of cross section models covering a wide energy range, the evaluation of specialized models with limited energy coverage and the appraisal of the capability of the examined cross section calculations to describe the photoelectric effect at the low energy end.

\section{A. Evaluation of Systematic Effects Related to Reference Data}

An analysis was performed prior to the proper validation process to establish whether semi-empirical cross sections could be

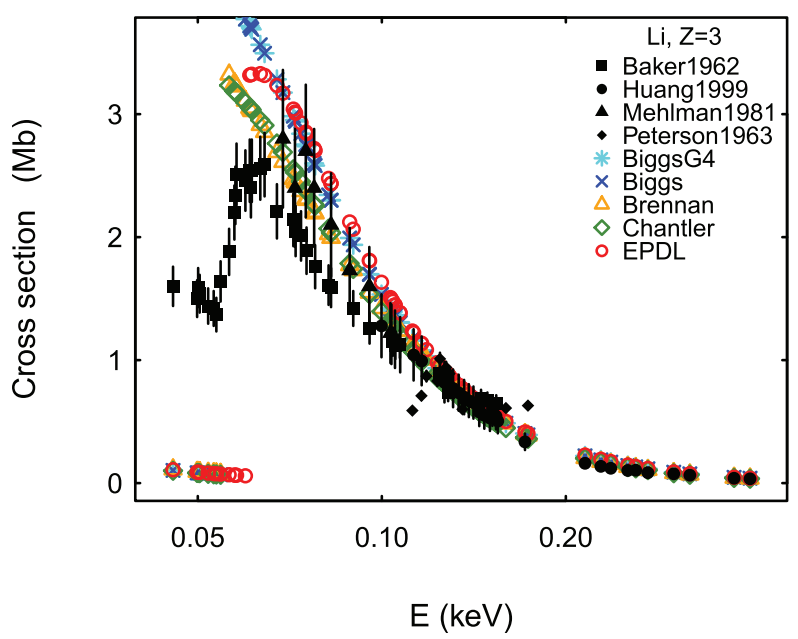

Fig. 3. Total photoionization cross section for $\mathrm{Z}=3$ as a function of photon energy.

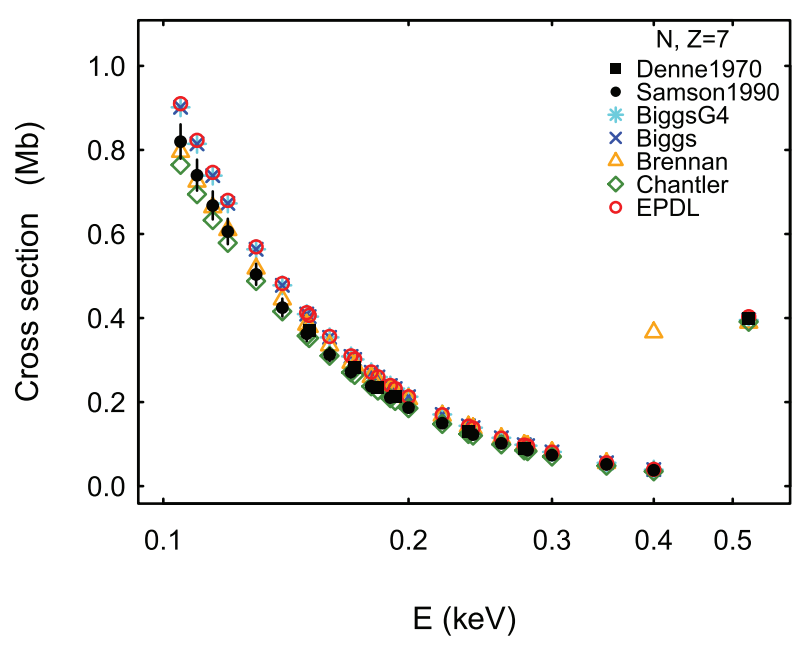

Fig. 4. Total photoionization cross section for $\mathrm{Z}=7$ as a function of photon energy.

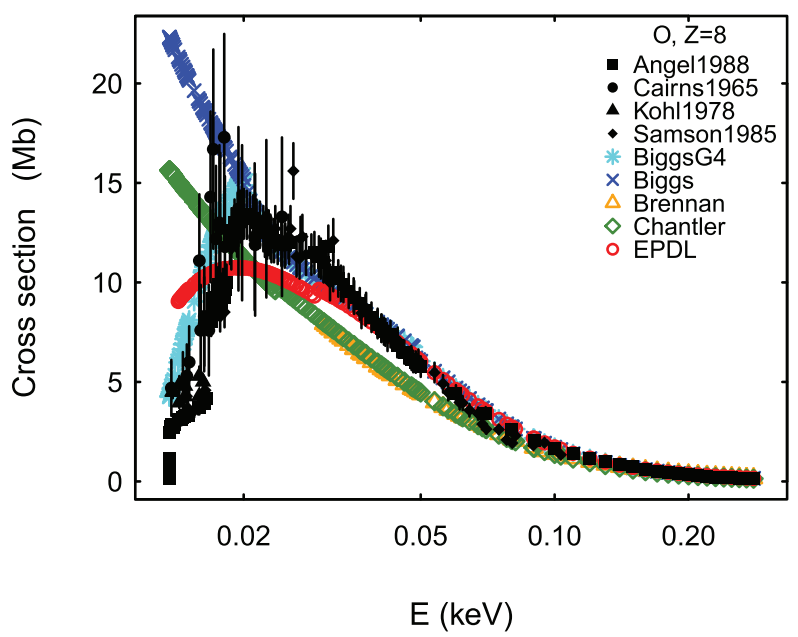

Fig. 5. Total photoionization cross section for $\mathrm{Z}=8$ as a function of photon energy. 


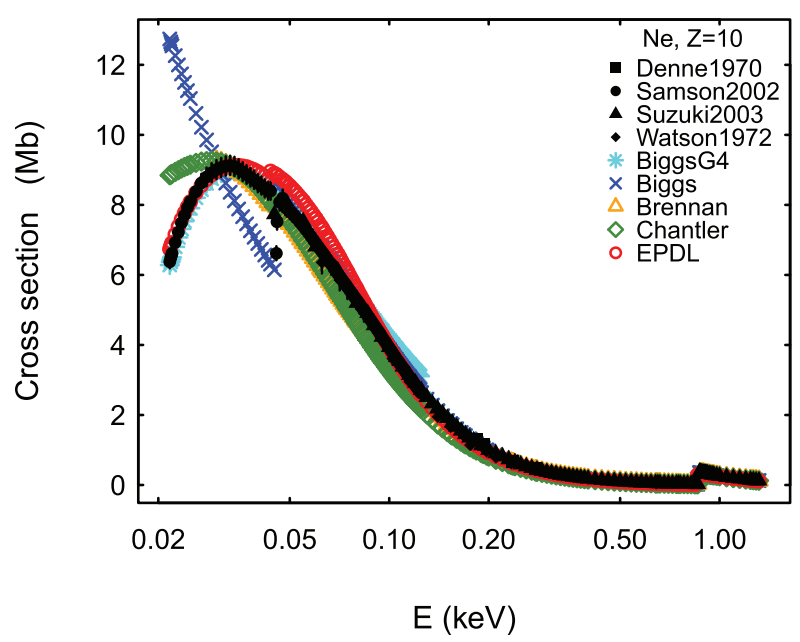

Fig. 6. Total photoionization cross section for $\mathrm{Z}=10$ as a function of photon energy.

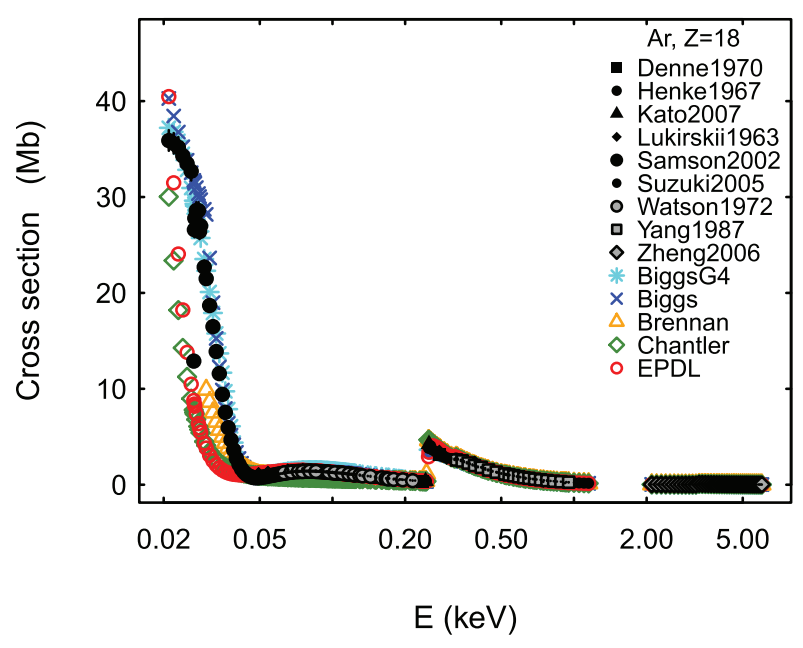

Fig. 7. Total photoionization cross section for $\mathrm{Z}=18$ as a function of photon energy.

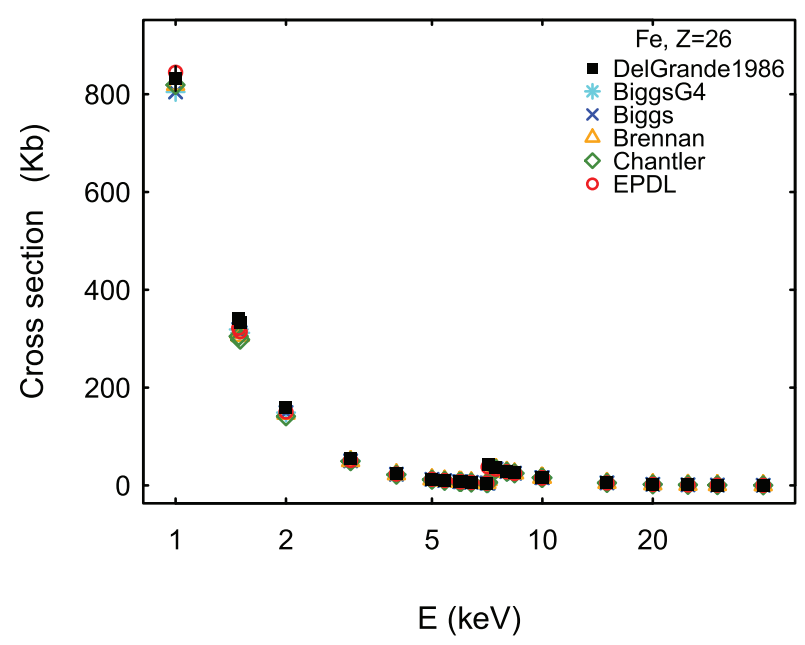

Fig. 8. Total photoionization cross section for $\mathrm{Z}=26$ as a function of photon energy.

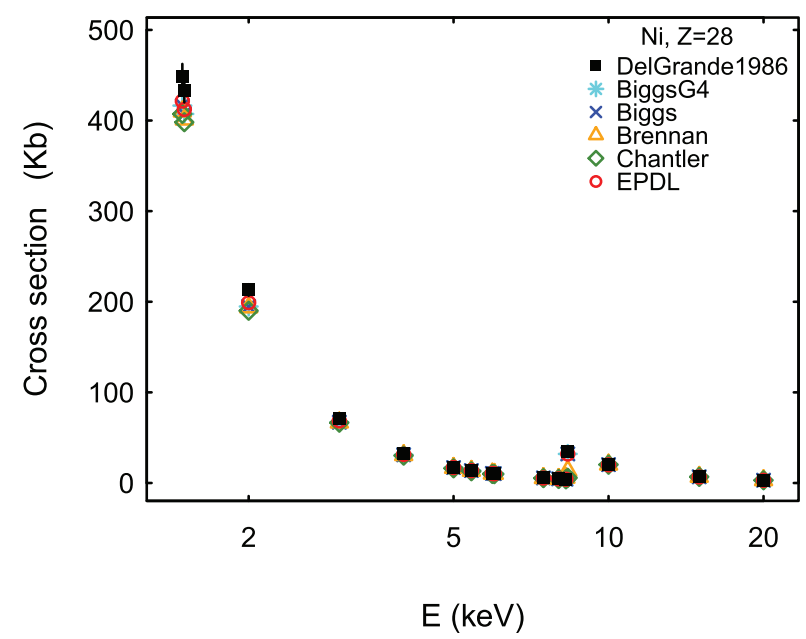

Fig. 9. Total photoionization cross section for $\mathrm{Z}=28$ as a function of photon energy.

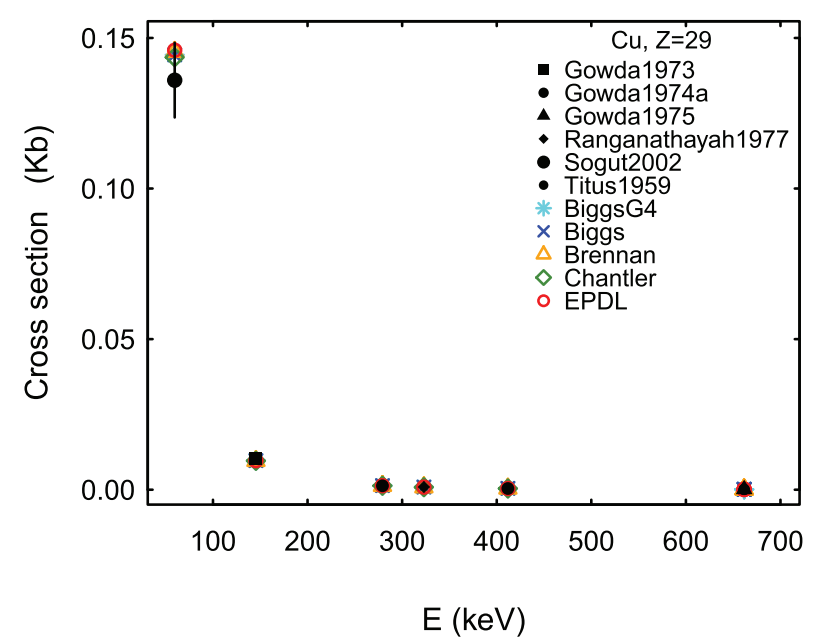

Fig. 10. Total photoionization cross section for $\mathrm{Z}=29$ as a function of photon energy.

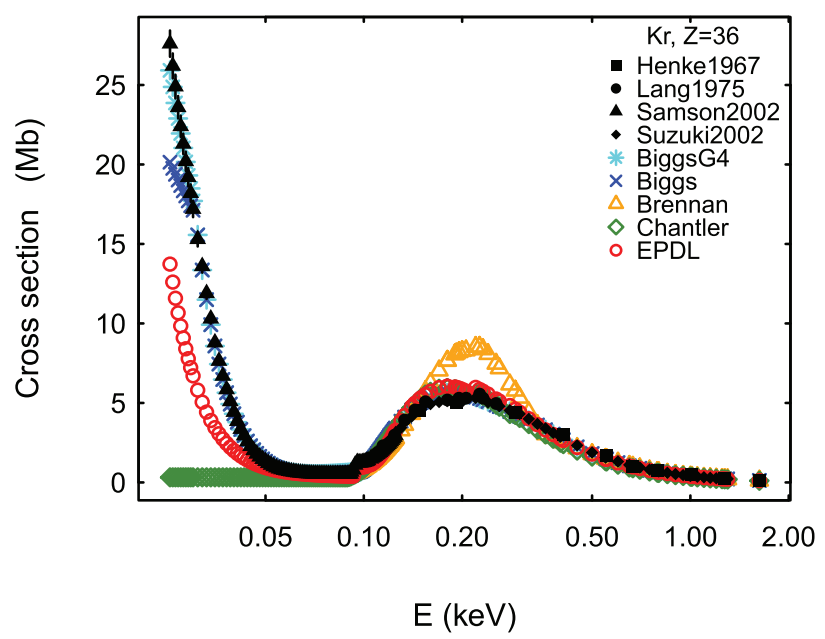

Fig. 11. Total photoionization cross section for $\mathrm{Z}=36$ as a function of photon energy. 


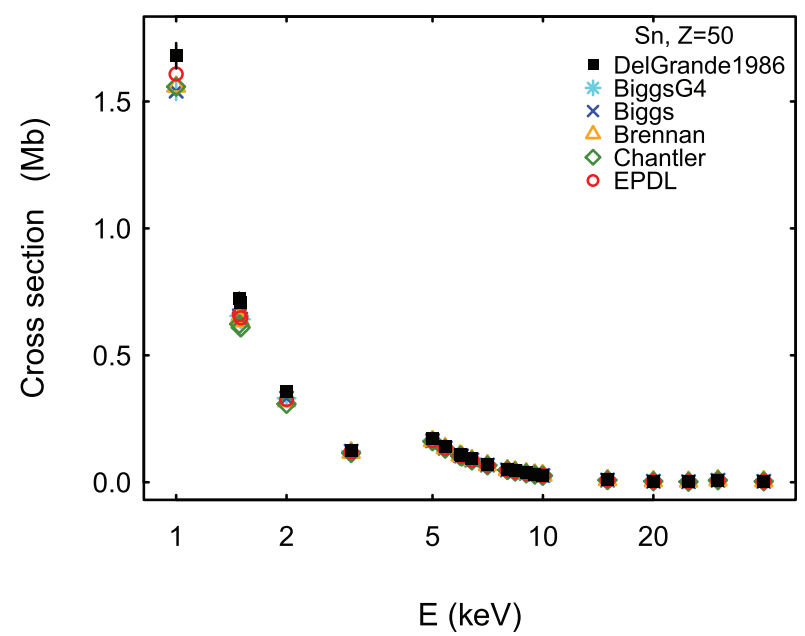

Fig. 12. Total photoionization cross section for $\mathrm{Z}=50$ as a function of photon energy.

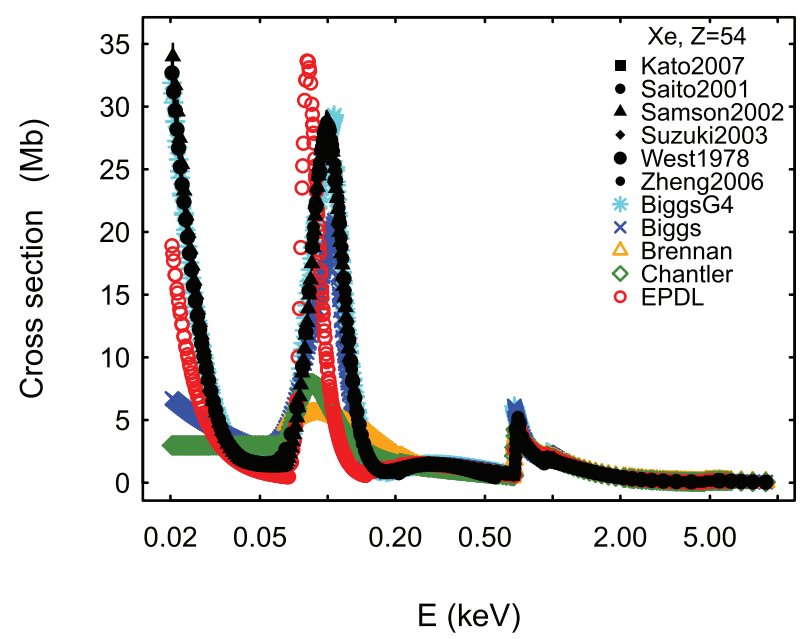

Fig. 13. Total photoionization cross section for $\mathrm{Z}=54$ as a function of photon energy.

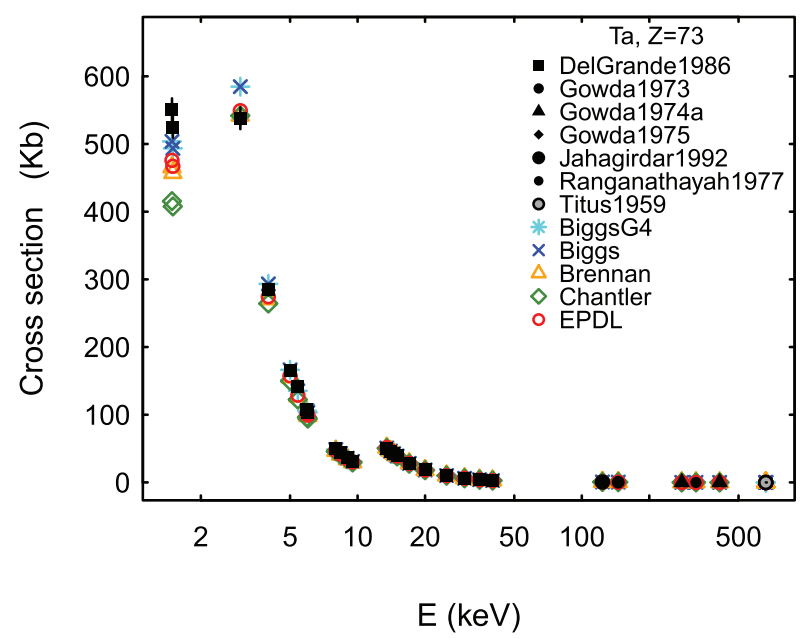

Fig. 14. Total photoionization cross section for $\mathrm{Z}=73$ as a function of photon energy.

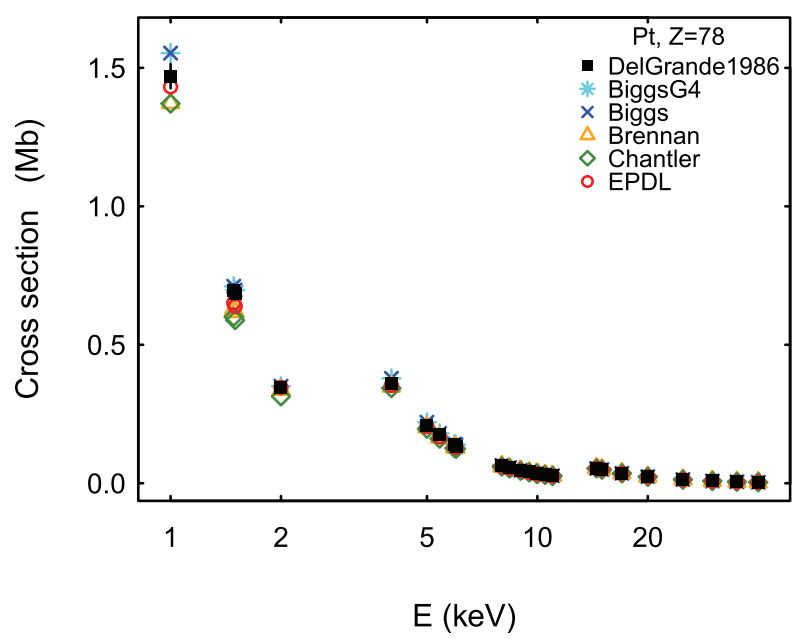

Fig. 15. Total photoionization cross section for $\mathrm{Z}=78$ as a function of photon energy.

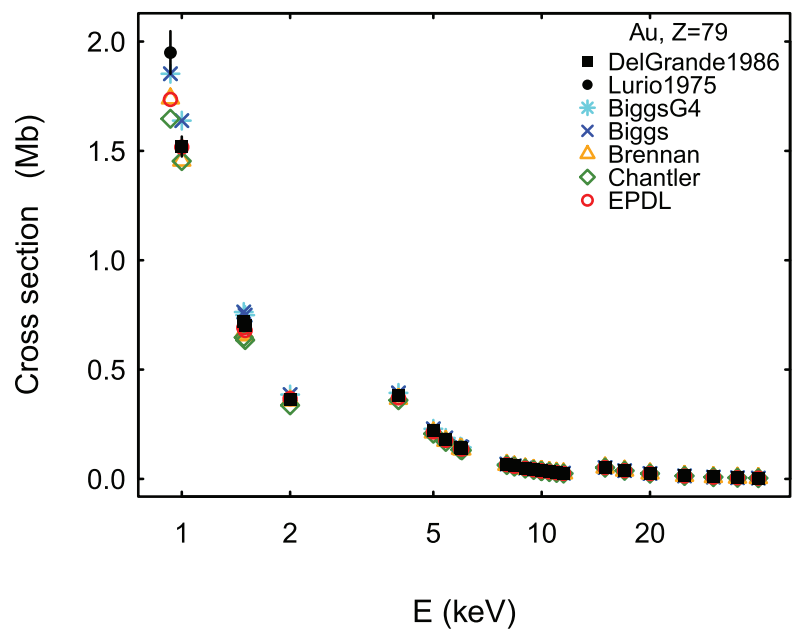

Fig. 16. Total photoionization cross section for $\mathrm{Z}=79$ as a function of photon energy.

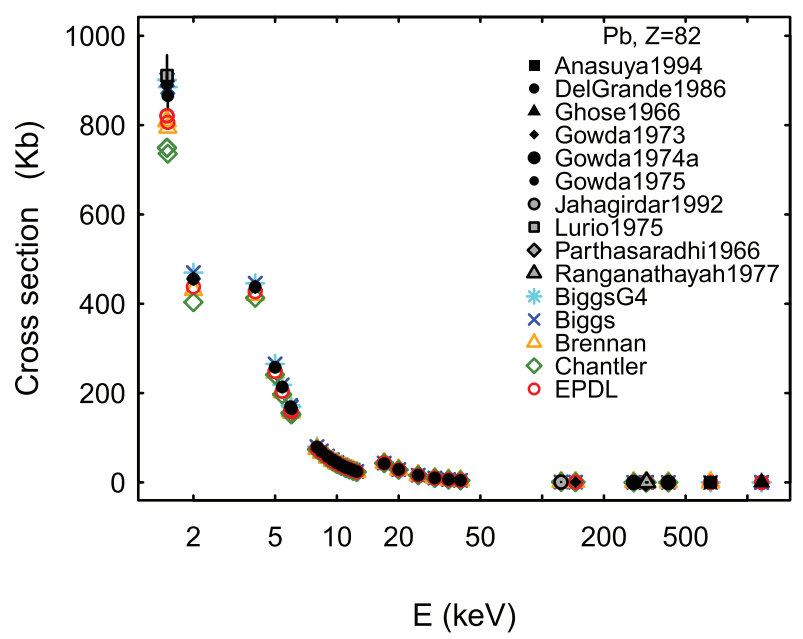

Fig. 17. Total photoionization cross section for $\mathrm{Z}=82$ as a function of photon energy. 


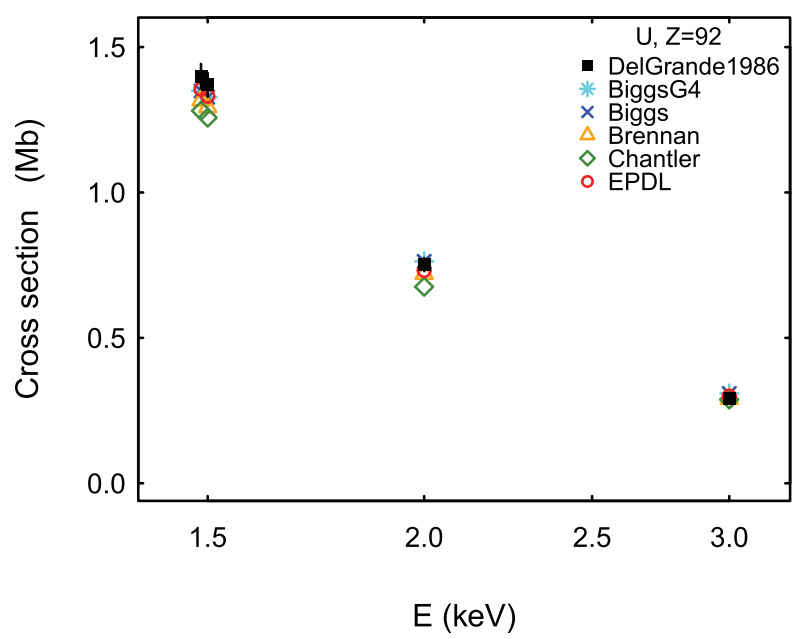

Fig. 18. Total photoionization cross section for $\mathrm{Z}=92$ as a function of photon energy.

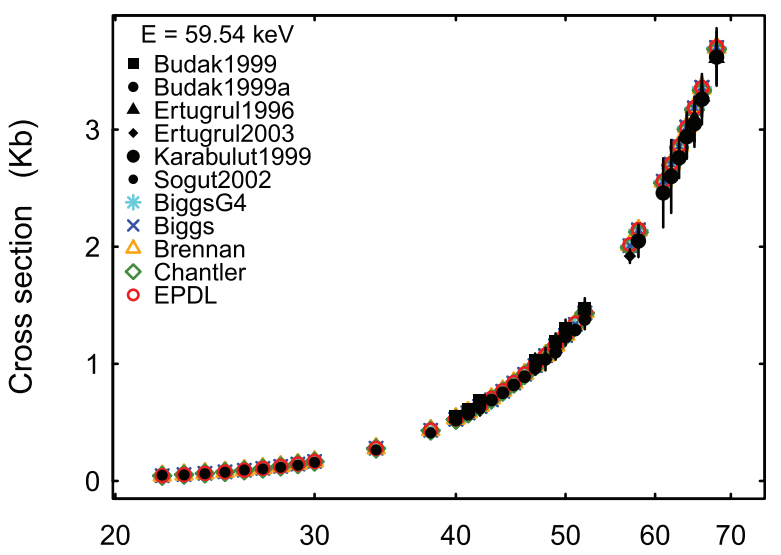

Atomic number

Fig. 19. Total photoionization cross section at $59.54 \mathrm{keV}$ as a function of the atomic number $\mathrm{Z}$.

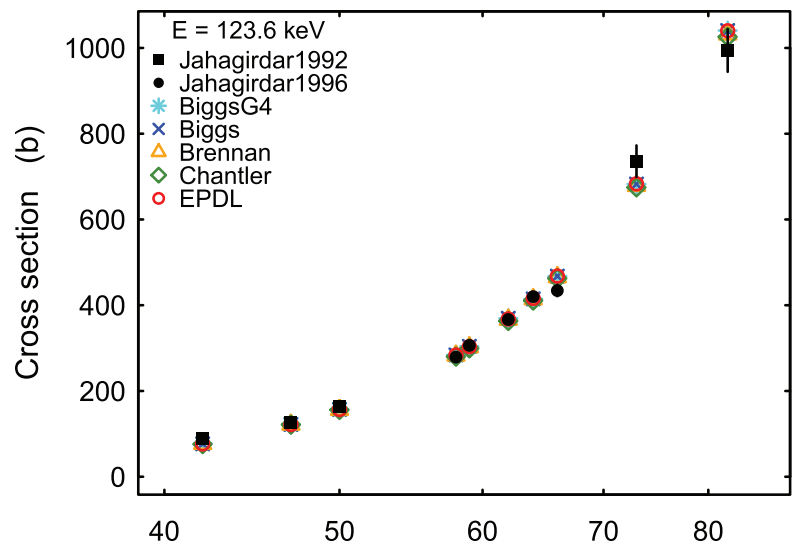

Atomic number

Fig. 20. Total photoionization cross section at $123.6 \mathrm{keV}$ as a function of the atomic number $\mathrm{Z}$.

used as a reference for comparison without introducing systematic effects. This evaluation concerns photon energies greater than $1 \mathrm{keV}$, since most of the semi-empirical data collected from

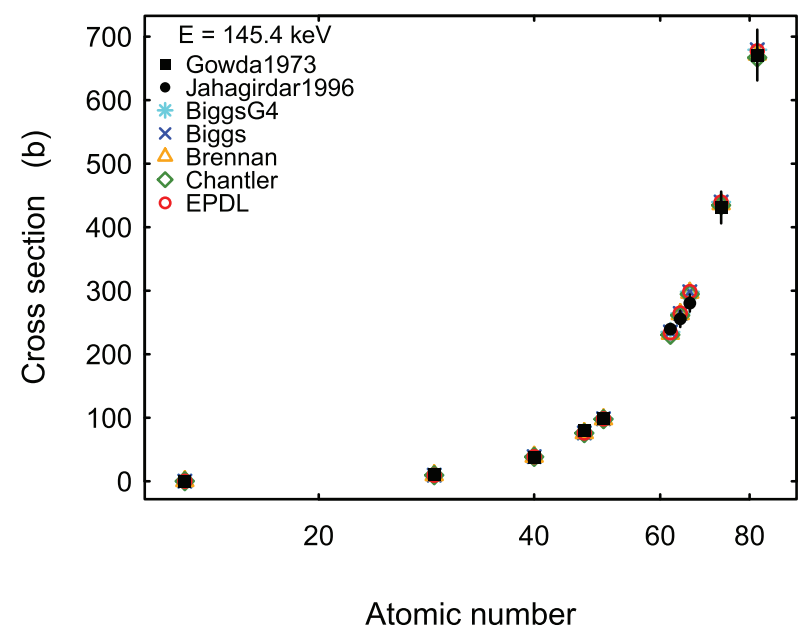

Fig. 21. Total photoionization cross section at $145.4 \mathrm{keV}$ as a function of the atomic number $\mathrm{Z}$.

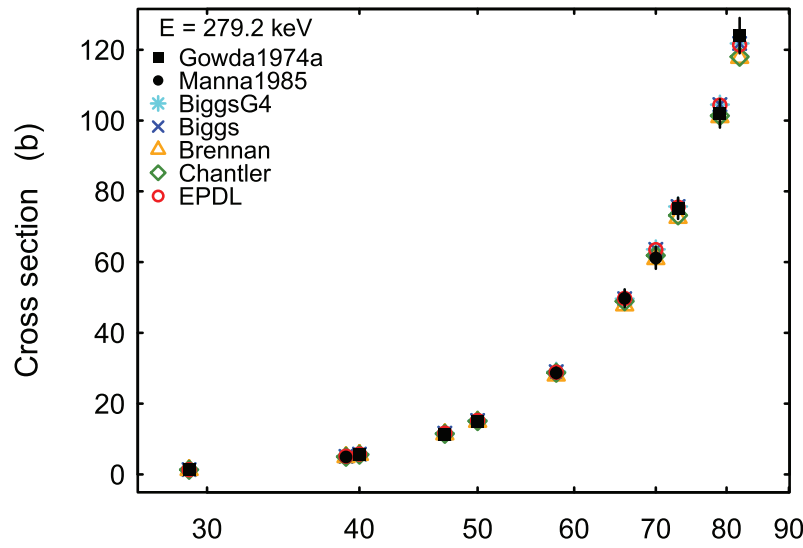

Atomic number

Fig. 22. Total photoionization cross section at $279.2 \mathrm{keV}$ as a function of the atomic number $\mathrm{Z}$.

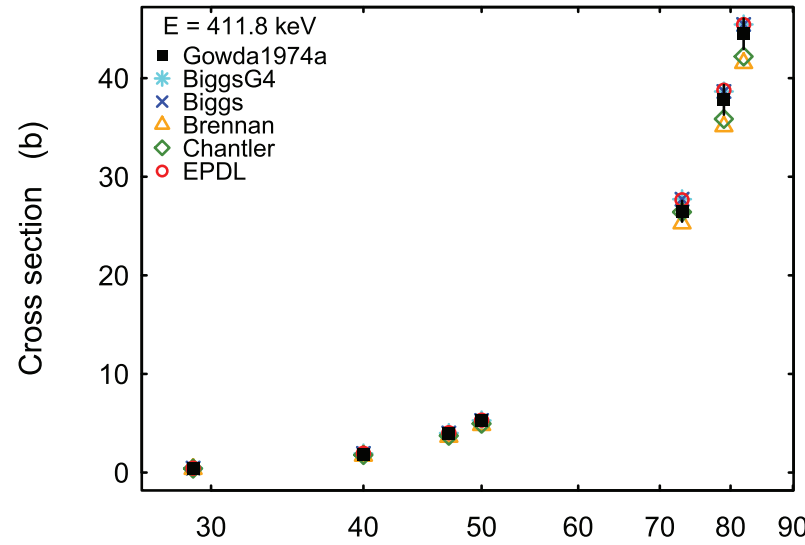

Atomic number

Fig. 23. Total photoionization cross section at $411.8 \mathrm{keV}$ as a function of the atomic number $\mathrm{Z}$.

the literature are above this energy; it involves cross section compilations that are applicable over the whole energy covered by experimental and semi-empirical data. 


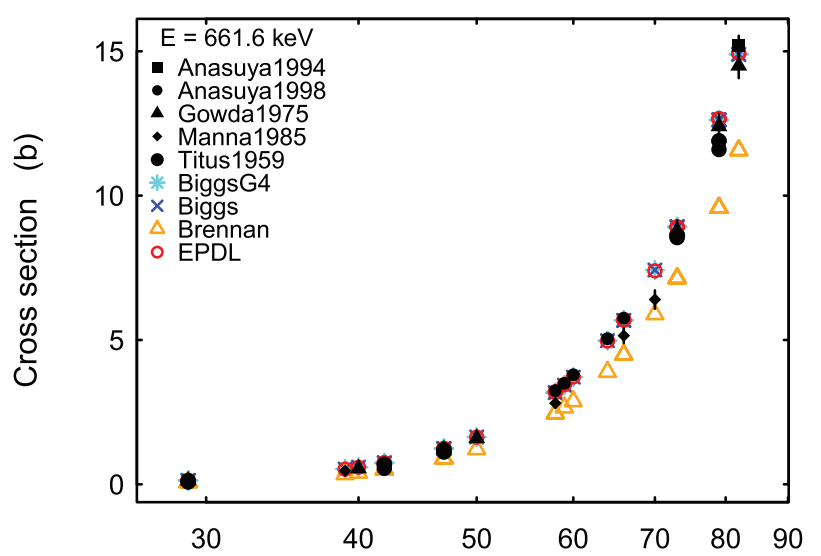

Atomic number

Fig. 24. Total photoionization cross section at $661.6 \mathrm{keV}$ as a function of the atomic number $\mathrm{Z}$.

TABLE IV

EFFICIENCY CALCULATED WITH RESPECT TO EXPERIMENTAL OR SEMI-EMPIRICAL REFERENCE DATA

\begin{tabular}{lccccccc}
\hline & \multicolumn{3}{c}{ Experimental } & & \multicolumn{3}{c}{ Semi-empirical } \\
\cline { 2 - 4 } \cline { 7 - 8 } Model & Pass & Fail & Efficiency & & Pass & Fail & Efficiency \\
\hline BiggsG4 & 33 & 9 & $0.79 \pm 0.06$ & & 171 & 128 & $0.57 \pm 0.03$ \\
Biggs & 33 & 9 & $0.79 \pm 0.06$ & & 171 & 128 & $0.57 \pm 0.03$ \\
EPDL & 36 & 6 & $0.86 \pm 0.05$ & & 180 & 119 & $0.60 \pm 0.03$ \\
Penelope & 36 & 6 & $0.86 \pm 0.05$ & & 180 & 119 & $0.60 \pm 0.03$ \\
PHOTX & 34 & 8 & $0.81 \pm 0.06$ & & 176 & 122 & $0.59 \pm 0.03$ \\
Scofield & 34 & 8 & $0.81 \pm 0.06$ & & 173 & 120 & $0.59 \pm 0.03$ \\
Storm & 30 & 12 & $0.71 \pm 0.07$ & & 175 & 123 & $0.59 \pm 0.03$ \\
XCOM & 35 & 7 & $0.83 \pm 0.06$ & & 178 & 120 & $0.60 \pm 0.03$ \\
\hline
\end{tabular}

TABLE V

TEST OF EQUIVALENT COMPATIBILITY OF CALCULATED TOTAL CROSS SECTIONS WITH EXPERIMENTAL OR SEMI-EMPIRICAL DATA

\begin{tabular}{lcccccc}
\hline & \multicolumn{6}{c}{ Test } \\
\cline { 2 - 7 } Model & Fisher & $\chi^{2}$ & Boschloo & Z-pooled & Santner & Barnard \\
\hline Biggs & 0.0110 & 0.0081 & 0.0099 & 0.0090 & 0.0090 & 0.0072 \\
EPDL & 0.0010 & 0.0013 & 0.0018 & 0.0023 & 0.0018 & 0.0008 \\
PHOTX & 0.0064 & 0.0066 & 0.0063 & 0.0075 & 0.0080 & 0.0056 \\
Scofield & 0.0063 & 0.0063 & 0.0061 & 0.0073 & 0.0079 & 0.0053 \\
Storm & 0.1319 & 0.1189 & 0.1219 & 0.1223 & 0.1278 & 0.1247 \\
XCOM & 0.0034 & 0.0032 & 0.0037 & 0.0041 & 0.0042 & 0.0024 \\
\hline
\end{tabular}

The efficiency at reproducing experimental and semiempirical reference data is reported in Table IV for all compilations covering the selected energy range. One observes that it is systematically lower, when semi-empirical data are considered as a reference in the comparison; the Wald-Wolfowitz test rejects the hypothesis of randomness of the sequence of results associated with experimental and semi-empirical references with 0.01 significance.

Categorical analysis performed over the compatibility of cross section calculations with experimental and semiempirical reference data confirms that the observed difference is statistically significant in all cases, with the exception of the Storm and Israel compilation. The p-values resulting from different tests over contingency tables are listed in Table V. The null hypothesis of equivalent compatibility with reference data
TABLE VI

P-VALUES FROM DIFFERENT TESTS COMPARING THE COMPATIBILITY WITH EXPERIMENT OF TOTAL CROSS SECTION MODELS WITH EXTENDED ENERGY COVERAGE AND EPDL, FOR ENERGIES ABOVE $1 \mathrm{KEV}$

\begin{tabular}{lcccccc}
\hline & \multicolumn{6}{c}{ Test } \\
\cline { 2 - 7 } Model & Fisher & $\chi^{2}$ & Boschloo & Z-pooled & Santner & Barnard \\
\hline BiggsG4 & 0.570 & 0.393 & 0.427 & 0.427 & 0.586 & 0.942 \\
Biggs & 0.570 & 0.393 & 0.427 & 0.427 & 0.586 & 0.942 \\
Penelope & 1 & 1 & 1 & 1 & 1 & 1 \\
PHOTX & 0.771 & 0.558 & 0.608 & 0.639 & 0.744 & 0.997 \\
Scofield & 0.771 & 0.558 & 0.608 & 0.639 & 0.744 & 0.997 \\
Storm & 0.183 & 0.111 & 0.130 & 0.122 & 0.230 & 0.138 \\
XCOM & 1 & 0.763 & 1 & 0.850 & 0.913 & 1 \\
\hline
\end{tabular}

is rejected by all tests with 0.01 significance in the comparison involving cross sections based on Scofield's 1973 calculations (EPDL, PHOTX, XCOM and Scofield's own tabulations). For the comparison concerning Biggs-Lighthill cross sections, the null hypothesis is rejected by all unconditional tests and by Pearson's $\chi^{2}$ tests, while it is not rejected by Fisher's exact test, which is known to be more conservative than unconditional tests. The insensitivity of the Storm and Israel model to the type of reference data to which it is compared is related to its overall lower compatibility with experiment reported in Table IV.

From these results one can infer that the use of semiempirical data as a reference in the comparison with photoelectric cross sections would introduce systematic effects in the validation process.

All the analyses reported in the following sections concern experimental data samples only.

\section{B. Evaluation of Total Cross Section Compilations with Wide Energy Coverage}

Some of the total cross section models considered in this study cover a wide energy range: those based on Scofield's 1973 non-relativistic calculations (including EPDL, PHOTX and XCOM compilations), Storm and Israel's compilation and Biggs-Lighthill's parameterization, both in its original form and in the modified version used by Geant 4 . Their extended applicability has contributed to their extensive use in particle transport codes.

General purpose Monte Carlo codes have traditionally handled photon interactions above $1 \mathrm{keV}$; extensions to lower energies have been included only relatively recently in some of them. The validation process has investigated the ability of these cross section compilations to reproduce experimental data as a function of energy, with special attention devoted to characterizing the behaviour at low energies, below $1 \mathrm{keV}$.

The efficiency of total cross section models applicable from $1 \mathrm{keV}$ up to the highest energy measurements included in the experimental sample (approximately $1.2 \mathrm{MeV}$ ) is reported in Table IV. The largest efficiency is achieved by EPDL (which is also the basis of Penelope's tabulations). Categorical tests based on contingency tables, summarized in Table VI, show that the differences in compatibility with experiment between the various models and EPDL are not statistically significant in this energy range. 
TABLE VII

EFFICIENCY BELOW 1 KEV OF TOTAL CROSS SECTION MODELS WITH EXTENDED COVERAGE

\begin{tabular}{cclccc}
\hline $\mathrm{E}_{\min }$ & $\mathrm{E}_{\max }$ & Model & Pass & Fail & Efficiency \\
\hline \multirow{3}{*}{$10 \mathrm{eV}$} & \multirow{2}{*}{$1 \mathrm{keV}$} & BiggsG4 & 12 & 31 & $0.28 \pm 0.07$ \\
& & Biggs & 13 & 30 & $0.30 \pm 0.07$ \\
& EPDL & 18 & 25 & $0.42 \pm 0.07$ \\
\hline \multirow{3}{*}{$100 \mathrm{eV}$} & \multirow{2}{*}{$1 \mathrm{keV}$} & BiggsG4 & 14 & 20 & $0.41 \pm 0.08$ \\
& & Biggs & 22 & 12 & $0.65 \pm 0.08$ \\
& EPDL & 23 & 11 & $0.68 \pm 0.08$ \\
\hline \multirow{3}{*}{$150 \mathrm{eV}$} & \multirow{2}{*}{$1 \mathrm{keV}$} & BiggsG4 & 14 & 12 & $0.54 \pm 0.09$ \\
& & Biggs & 17 & 9 & $0.65 \pm 0.09$ \\
& & EPDL & 19 & 7 & $0.73 \pm 0.08$ \\
\hline \multirow{2}{*}{$250 \mathrm{eV}$} & \multirow{2}{*}{$1 \mathrm{keV}$} & BiggsG4 & 14 & 7 & $0.67 \pm 0.10$ \\
& & Biggs & 14 & 7 & $0.67 \pm 0.10$ \\
& EPDL & 15 & 6 & $0.71 \pm 0.09$ \\
\hline
\end{tabular}

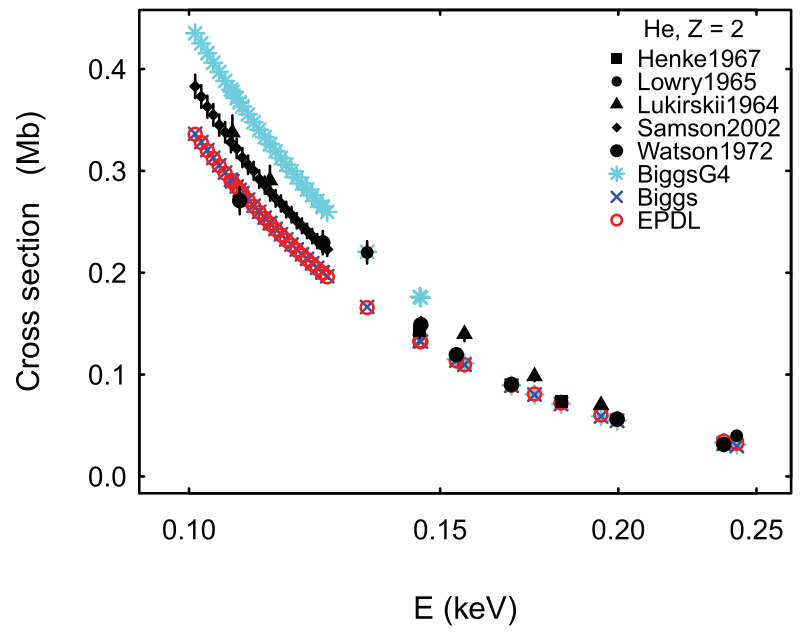

Fig. 25. Total photoionization cross section for helium as a function of photon energy, above $100 \mathrm{eV}$ : original and modified Biggs-Lighthill parameterizations exhibit different behavior with respect to experimental data.

One observes some small differences in Table IV regarding the efficiencies of cross section compilations derived from Scofield's 1973 calculations. They are due to differences in the granularity of the energy grid at which cross sections are tabulated, which affects the precision of interpolation.

Only EPDL and Biggs-Lighthill's parameterization cover the whole energy range corresponding to the experimental data sample, including energies below $1 \mathrm{keV}$; their efficiencies are reported in Table VII for a series of low energy intervals. All models exhibit low efficiencies below approximately $100 \mathrm{eV}$; above this energy the efficiencies of cross sections based on EPDL and on the original Biggs-Lighthill's parameterization appear quite stable (compatible with statistical uncertainties) and similar, although EPDL ones are always larger.

The modified coefficients of Biggs and Lighthill's parameterization implemented in Geant 4 do not appear to improve the compatibility with experiment of the calculated cross sections; discrepancies with respect to experimental data are qualitatively visible in Figs. 1 and 25-28. Cross sections calculated with the original coefficients appear unable to reproduce experimental data consistently in the very low energy range, below a few tens of eV: a few examples are shown in Figs. 29-32.

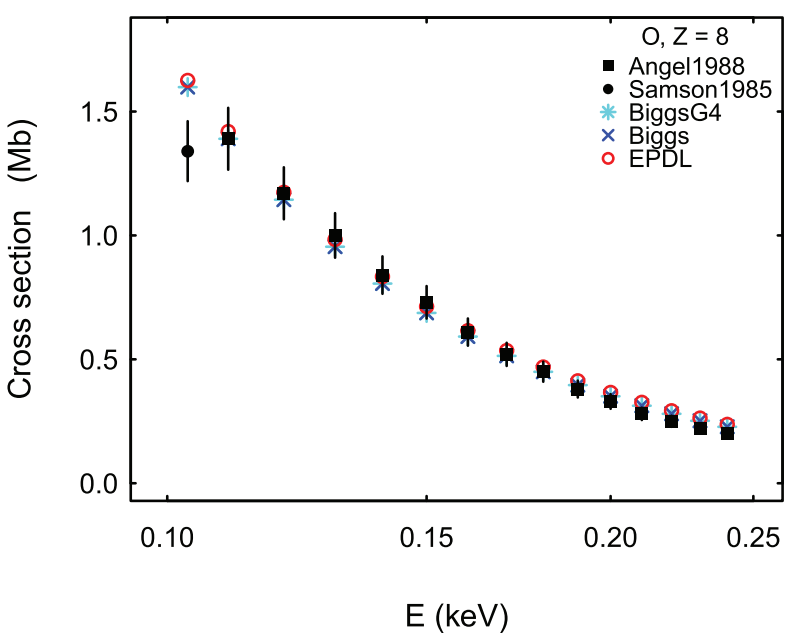

Fig. 26. Total photoionization cross section for oxygen as a function of photon energy, above $100 \mathrm{eV}$ : original and modified Biggs-Lighthill parameterizations exhibit different behavior with respect to experimental data.

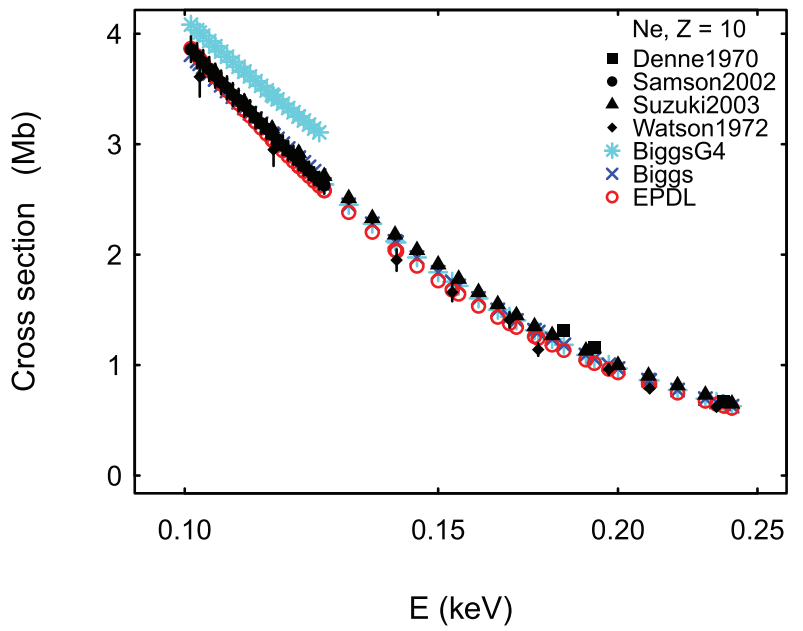

Fig. 27. Total photoionization cross section for neon as a function of photon energy, above $100 \mathrm{eV}$ : original and modified Biggs-Lighthill parameterizations exhibit different behavior with respect to experimental data.

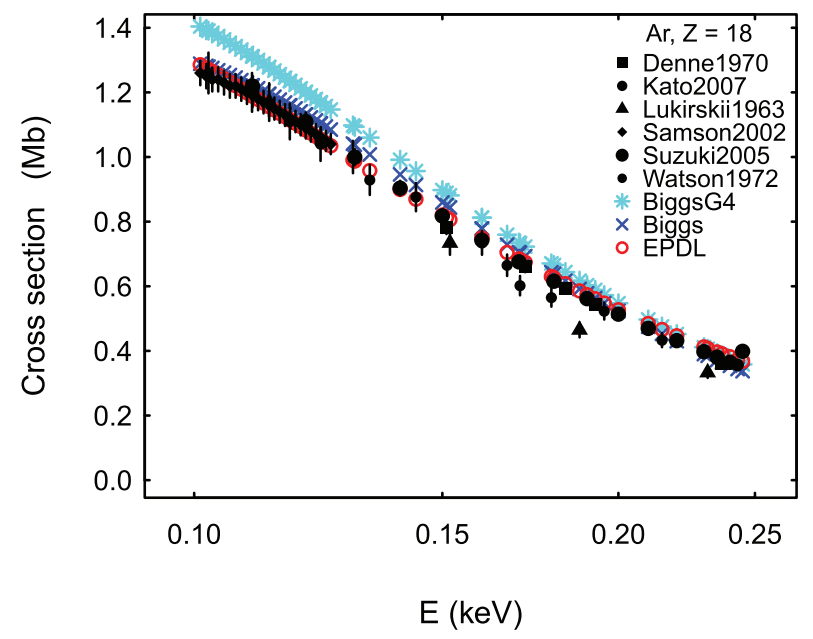

Fig. 28. Total photoionization cross section for argon as a function of photon energy, above $100 \mathrm{eV}$ : original and modified Biggs-Lighthill parameterizations exhibit different behavior with respect to experimental data. 


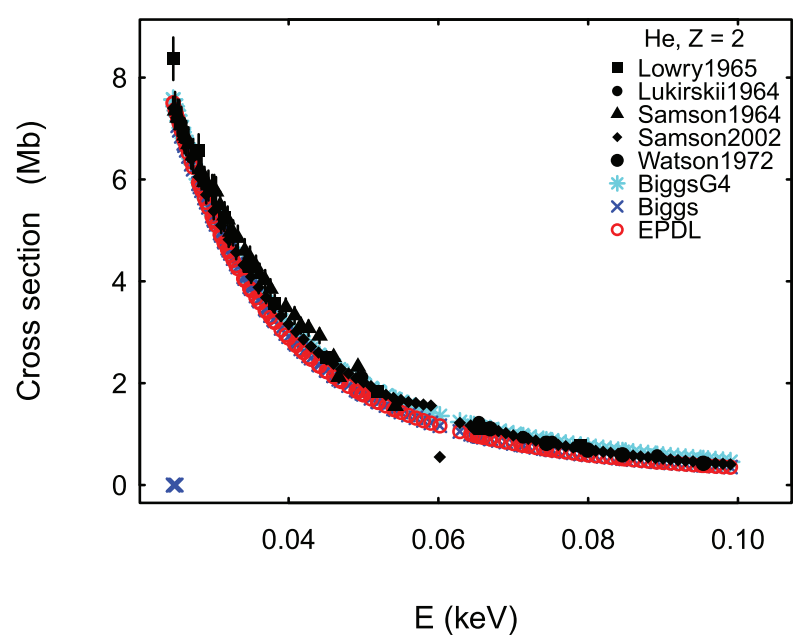

Fig. 29. Total photoionization cross section for helium as a function of photon energy, below $100 \mathrm{eV}$ : original and modified Biggs-Lighthill parameterizations exhibit different behavior with respect to experimental data.

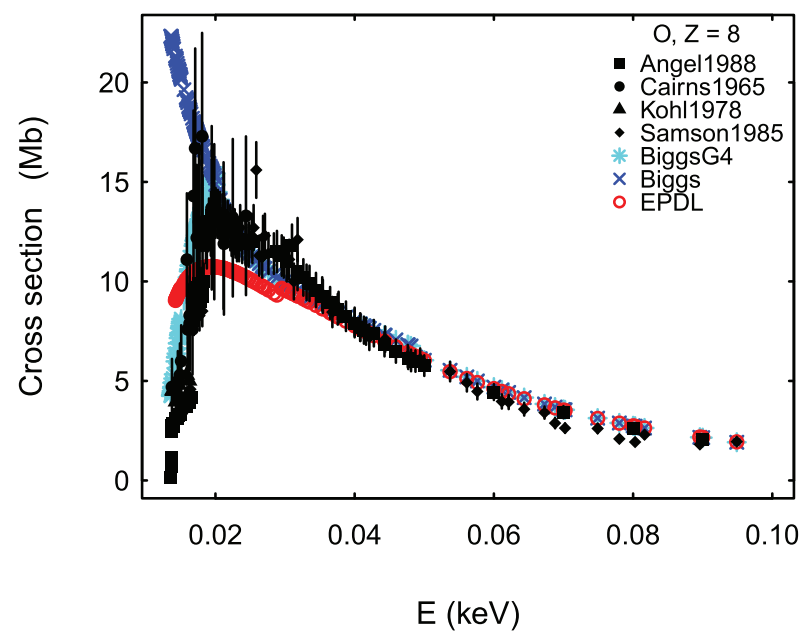

Fig. 30. Total photoionization cross section for oxygen as a function of photon energy, below $100 \mathrm{eV}$ : original and modified Biggs-Lighthill parameterizations exhibit different behavior with respect to experimental data.

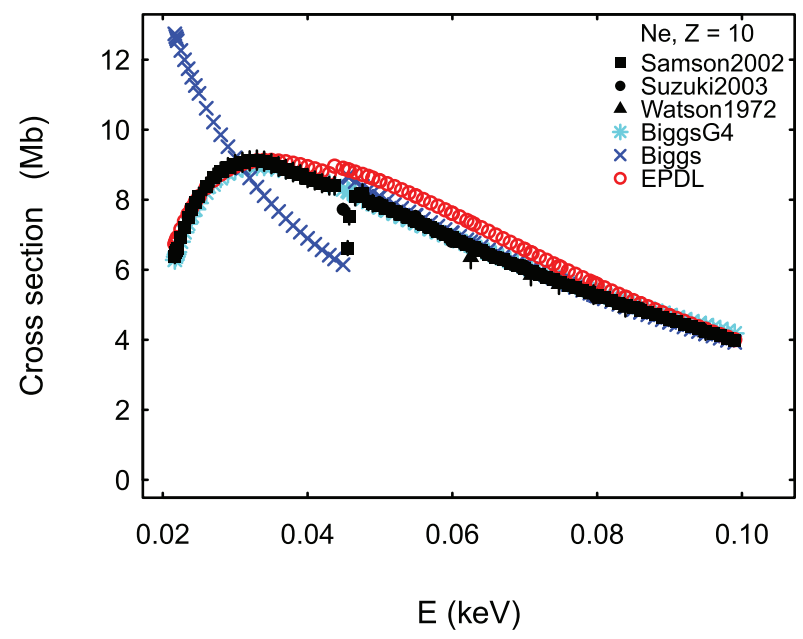

Fig. 31. Total photoionization cross section for neon as a function of photon energy, below $100 \mathrm{eV}$ : original and modified Biggs-Lighthill parameterizations exhibit different behavior with respect to experimental data.

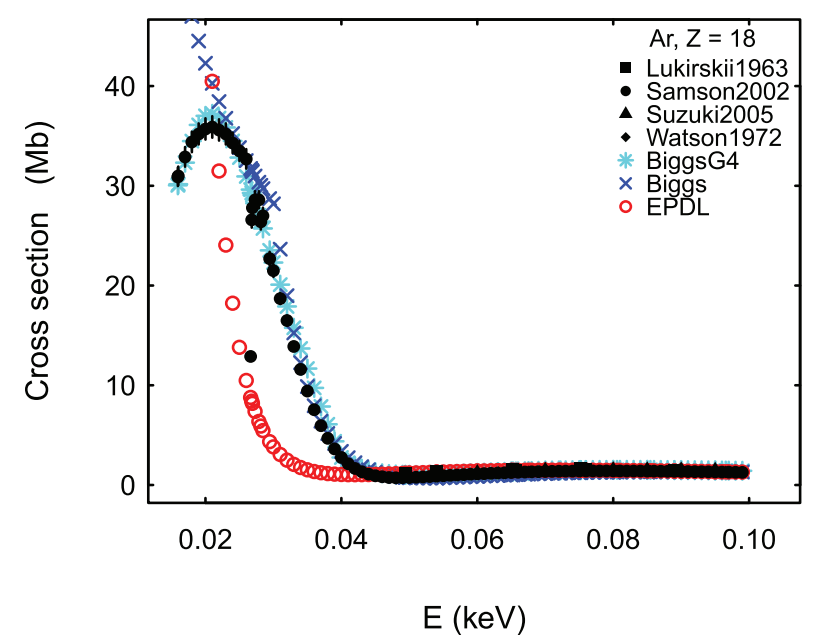

Fig. 32. Total photoionization cross section for argon as a function of photon energy, below $100 \mathrm{eV}$ : original and modified Biggs-Lighthill parameterizations exhibit different behavior with respect to experimental data.

TABLE VIII

EFFICIENCY OF TOTAL CROSS SECTIONS BASED ON ORIGINAL AND MODIFIED BIGGS-LIGHTHILL PARAMETERIZATION, LIMITED TO THE TEST CASES WHERE THEY DIFFER

\begin{tabular}{lccccccc}
\hline & \multicolumn{3}{c}{$\mathrm{E}<100 \mathrm{eV}$} & & \multicolumn{3}{c}{$\mathrm{E} \geq 100 \mathrm{eV}$} \\
\cline { 2 - 4 } \cline { 7 - 8 } Model & Pass & Fail & Efficiency & & Pass & Fail & Efficiency \\
\hline BiggsG4 & 6 & 16 & $0.27 \pm 0.09$ & & 1 & 13 & $0.07 \pm 0.07$ \\
Biggs & 2 & 20 & $0.09 \pm 0.07$ & & 10 & 4 & $0.71 \pm 0.12$ \\
EPDL & 5 & 17 & $0.23 \pm 0.09$ & & 11 & 3 & $0.79 \pm 0.11$ \\
RTAB & 7 & 15 & $0.32 \pm 0.10$ & & 11 & 3 & $0.79 \pm 0.11$ \\
\hline
\end{tabular}

TABLE IX

COMPARISON OF THE COMPATIBILITY WITH EXPERIMENT OF TOTAL CROSS SECTIONS BASED ON ORIGINAL AND MODIFIED BIGGS-LIGHTHILL PARAMETERIZATION

\begin{tabular}{lcc}
\hline Test & p-value, $\mathrm{E}<100 \mathrm{eV}$ & p-value, $\mathrm{E} \geq 100 \mathrm{eV}$ \\
\hline Fisher & 0.240 & 0.0013 \\
Z-pooled & 0.137 & 0.0004 \\
Boschloo & 0.174 & 0.0004 \\
Santner & 0.259 & 0.0004 \\
Barnard & 0.223 & 0.0004 \\
\hline
\end{tabular}

A dedicated statistical analysis was performed to quantify whether the difference in compatibility with experiment between the two parameterizations is significant. For this purpose, the data sample was limited to test cases where the two cross section calculations produce different values at the same energy of an experimental measurement; these test cases concern only noble gases, oxygen and hydrogen. Two test cases were considered: one involving energies below $100 \mathrm{eV}$ and one concerning measurements above or equal to $100 \mathrm{eV}$. The corresponding efficiencies are reported in Table VIII, along with EPDL and RTAB results: the original Biggs and Lighthill parameterization, as well as EPDL and RTAB compilations, exhibit low efficiencies in the lower energy range, while the efficiency of EPDL, RTAB and the original Biggs and Lighthill parameterization achieve substantially better compatibility with experiment above $100 \mathrm{eV}$. It is worth recalling that this test 
TABLE $X$

P-VALUES CONCERNING THE COMPARISON OF THE COMPATIBILITY WITH EXPERIMENT OF SPECIFIC TOTAL CROSS SECTION MODELS AND EPDL

\begin{tabular}{|c|c|c|c|c|c|c|c|c|}
\hline \multirow[b]{2}{*}{ Model } & \multicolumn{2}{|c|}{ Energy range } & \multicolumn{6}{|c|}{ p-values from tests over contingency tables } \\
\hline & $\mathrm{E}_{\min }$ & $\mathrm{E}_{\max }$ & Fisher & $\chi^{2}$ & Boschloo & Z-pooled & Santner & Barnard \\
\hline \multirow{2}{*}{ Brennan } & $30 \mathrm{eV}$ & $700 \mathrm{keV}$ & 0.006 & 0.004 & 0.005 & 0.004 & 0.004 & 0.004 \\
\hline & $150 \mathrm{eV}$ & $700 \mathrm{keV}$ & 0.004 & 0.002 & 0.003 & 0.003 & 0.003 & 0.002 \\
\hline \multirow{2}{*}{ Chantler } & $10 \mathrm{eV}$ & $433 \mathrm{keV}$ & 0.0097 & 0.006 & 0.008 & 0.008 & 0.007 & 0.00999 \\
\hline & $150 \mathrm{eV}$ & $433 \mathrm{keV}$ & 0.006 & 0.003 & 0.005 & 0.005 & 0.006 & 0.003 \\
\hline Ebel & $1 \mathrm{keV}$ & $300 \mathrm{keV}$ & 1 & 0.739 & 0.836 & 0.834 & 0.897 & 0.994 \\
\hline Elam & $100 \mathrm{eV}$ & $1 \mathrm{MeV}$ & 0.124 & 0.084 & 0.093 & 0.093 & 0.133 & 0.087 \\
\hline \multirow{2}{*}{ Henke } & $10 \mathrm{eV}$ & $30 \mathrm{keV}$ & 0.151 & 0.101 & 0.109 & 0.110 & 0.128 & 0.108 \\
\hline & $150 \mathrm{eV}$ & $30 \mathrm{keV}$ & 0.049 & 0.027 & 0.031 & 0.031 & 0.032 & 0.030 \\
\hline McMaster & $1 \mathrm{keV}$ & $700 \mathrm{keV}$ & 0.041 & 0.033 & 0.033 & 0.040 & 0.071 & 0.039 \\
\hline \multirow{2}{*}{ RTAB } & $10 \mathrm{eV}$ & $300 \mathrm{keV}$ & 1 & 0.843 & 0.828 & 0.917 & 0.920 & 0.997 \\
\hline & $150 \mathrm{eV}$ & $300 \mathrm{keV}$ & 1 & 0.793 & 1 & 0.880 & 0.903 & 0.870 \\
\hline Storm-Israel & $1 \mathrm{keV}$ & $100 \mathrm{MeV}$ & 0.183 & 0.111 & 0.130 & 0.122 & 0.230 & 0.138 \\
\hline Veigele & $100 \mathrm{eV}$ & $1 \mathrm{MeV}$ & 1 & 0.843 & 0.828 & 0.917 & 0.920 & 0.997 \\
\hline XCOM-DB & $1 \mathrm{keV}$ & $500 \mathrm{keV}$ & 0.371 & 0.232 & 0.273 & 0.273 & 0.403 & 0.371 \\
\hline
\end{tabular}

concerns data below $1 \mathrm{keV}$, since the coefficients of the two parameterizations differ only in the low energy range.

The results of the analysis of these categorical data, summarized in Table IX, show that above $100 \mathrm{eV}$ the null hypothesis of equivalent compatibility with experiment of the two parameterizations is rejected with 0.01 significance by all the tests applied to the associated contingency table, while it is not rejected below $100 \mathrm{eV}$.

These results suggest reverting to the original coefficients of Biggs-Lighthill's parameterization in Geant4 for improved accuracy of the physics models that use cross sections based on it, at least at energies above a few tens of $\mathrm{eV}$.

\section{Evaluation of Specific Total Cross Section Compilations}

This investigation addresses the issue of whether specialized calculation models produce more accurate results than extensive cross section compilations in the limited energy range they cover and therefore deserve to partially replace the extensive cross section compilations currently used in general purpose Monte Carlo codes.

The total cross section compilations listed in Table IV cover different energy ranges, therefore the evaluation of their intrinsic capabilities requires specific validation tests limited to the energy range of applicability of each of them.

Efficiencies pertinent to each cross section model are reported in detail in the following subsections; they are calculated over the subset of the experimental data sample consistent with the applicability of each model. The associated tables show the model especially addressed in each test in italic.

The compatibility with experiment of each specialized cross section model has been compared with that of EPDL, which is the compilation with the highest efficiency in Table IV, limited to the energy range where the model subject to evaluation is applicable. The results of the statistical analysis that addresses this issue are summarized in Table $\mathrm{X}$; they are discussed in detail in the following subsections.

1) Brennan and Cowan's Cross Sections: these cross sections cover the energy range between $30 \mathrm{eV}$ and $700 \mathrm{keV}$, and are limited to atomic number greater than 2. The efficiencies listed in Table XI show lower capability of Brennan
TABLE XI

TOTAL CROSS SECTIONS: EFFICIENCY BETWEEN 1 KEV AND $300 \mathrm{KEV}$

\begin{tabular}{lccccccc}
\hline & \multicolumn{3}{c}{$\mathrm{E}_{\text {min }}=10 \mathrm{eV}$} & & \multicolumn{3}{c}{$\mathrm{E}_{\min }=150 \mathrm{eV}$} \\
\cline { 2 - 4 } \cline { 7 - 9 } Model & Pass & Fail & Efficiency & & Pass & Fail & Efficiency \\
\hline BiggsG4 & 38 & 23 & $0.62 \pm 0.06$ & & 37 & 16 & $0.70 \pm 0.06$ \\
Biggs & 38 & 23 & $0.62 \pm 0.06$ & & 40 & 13 & $0.75 \pm 0.06$ \\
Brennan & 26 & 35 & $0.43 \pm 0.06$ & & 27 & 26 & $0.51 \pm 0.07$ \\
EPDL & 42 & 19 & $0.69 \pm 0.06$ & & 42 & 11 & $0.79 \pm 0.06$ \\
\hline
\end{tabular}

TABLE XII

TOTAL CROSS SECTIONS: EFFICIENCY BETWEEN 100 EV AND 1 MeV

\begin{tabular}{lccccccc}
\hline & \multicolumn{3}{c}{$\mathrm{E}_{\min }=10 \mathrm{eV}$} & & \multicolumn{3}{c}{$\mathrm{E}_{\min }=150 \mathrm{eV}$} \\
\cline { 2 - 4 } \cline { 7 - 9 } Model & Pass & Fail & Efficiency & & Pass & Fail & Efficiency \\
\hline BiggsG4 & 34 & 24 & $0.50 \pm 0.06$ & & 36 & 15 & $0.71 \pm 0.06$ \\
Biggs & 35 & 33 & $0.51 \pm 0.06$ & & 39 & 12 & $0.76 \pm 0.06$ \\
Chantler & 24 & 44 & $0.35 \pm 0.06$ & & 27 & 24 & $0.53 \pm 0.07$ \\
EPDL & 40 & 28 & $0.59 \pm 0.06$ & & 41 & 10 & $0.80 \pm 0.06$ \\
\hline
\end{tabular}

and Cowan's cross sections with respect to other compilations applicable to this energy range.

According to the results of the categorical analysis listed in Table X, the compatibility with experiment of these cross sections and EPDL ones is rejected with 0.01 significance, both over the entire energy interval of applicability and excluding test cases in the lower energy end.

From this analysis one can infer that Brennan and Cowan's calculations do not achieve greater accuracy than Scofield's 1973 non-relativistic ones tabulated in EPDL.

2) Chantler's Total Cross Sections: these calculations cover energies between $10 \mathrm{eV}$ and $433 \mathrm{keV}$. The efficiency of the cross section models applicable in this energy range is reported in Table XII: it is lower for Chantler's cross sections than for other options applicable to this energy interval. The p-values listed in Table X show that the hypothesis of equivalent compatibility with experiment of Chantler's cross sections with respect to EPDL ones is rejected by all tests, both over the entire energy interval and excluding the lower energy end.

3) Ebel's Total Cross Sections: this parameterization is applicable to energies between $1 \mathrm{keV}$ and $300 \mathrm{keV}$. The efficiencies listed in Table XIII, which are calculated for all 
TABLE XIII

TOTAL CROSS SECTIONS: EFFICIENCY BETWEEN 1 KEV AND $300 \mathrm{KEV}$

\begin{tabular}{lccc}
\hline Model & Pass & Fail & Efficiency \\
\hline BiggsG4 & 22 & 8 & $0.73 \pm 0.08$ \\
Biggs & 22 & 8 & $0.73 \pm 0.08$ \\
Brennan & 19 & 11 & $0.63 \pm 0.08$ \\
Chantler & 17 & 13 & $0.57 \pm 0.09$ \\
Ebel & 24 & 6 & $0.80 \pm 0.07$ \\
Elam & 23 & 7 & $0.77 \pm 0.08$ \\
EPDL & 25 & 5 & $0.83 \pm 0.07$ \\
PHOTX & 23 & 7 & $0.77 \pm 0.08$ \\
Scofield & 23 & 7 & $0.77 \pm 0.08$ \\
Storm & 19 & 11 & $0.63 \pm 0.08$ \\
Veigele & 17 & 13 & $0.57 \pm 0.09$ \\
XCOM-DB & 21 & 9 & $0.70 \pm 0.08$ \\
XCOM & 24 & 6 & $0.80 \pm 0.08$ \\
\hline
\end{tabular}

TABLE XIV

TOTAL CROSS SECTIONS: EFFICIENCY BETWEEN 100 EV AND $1 \mathrm{MEV}$

\begin{tabular}{lccc}
\hline Model & Pass & Fail & Efficiency \\
\hline BiggsG4 & 40 & 24 & $0.63 \pm 0.06$ \\
Biggs & 48 & 16 & $0.75 \pm 0.05$ \\
Elam & 40 & 24 & $0.63 \pm 0.06$ \\
EPDL & 49 & 15 & $0.77 \pm 0.05$ \\
Veigele & 30 & 34 & $0.47 \pm 0.06$ \\
\hline
\end{tabular}

TABLE XY

TOTAL CROSS SECTIONS: EFFICIENCY BETWEEN 10/150 EV AND $30 \mathrm{KEV}$

\begin{tabular}{lcccccccc}
\hline & \multicolumn{3}{c}{$\mathrm{E}_{\text {min }}=10 \mathrm{eV}$} & & \multicolumn{3}{c}{$\mathrm{E}_{\min }=150 \mathrm{eV}$} \\
\cline { 2 - 4 } \cline { 7 - 9 } Model & Pass & Fail & Efficiency & & Pass & Fail & Efficiency \\
\hline BiggsG4 & 18 & 33 & $0.35 \pm 0.07$ & & 20 & 14 & $0.59 \pm 0.08$ \\
Biggs & 19 & 32 & $0.37 \pm 0.07$ & & 23 & 11 & $0.68 \pm 0.08$ \\
Chantler & 7 & 44 & $0.14 \pm 0.05$ & & 10 & 24 & $0.29 \pm 0.08$ \\
EPDL & 23 & 28 & $0.45 \pm 0.07$ & & 24 & 10 & $0.71 \pm 0.08$ \\
Henke & 15 & 36 & $0.29 \pm 0.06$ & & 15 & 19 & $0.44 \pm 0.08$ \\
RTAB & 24 & 27 & $0.47 \pm 0.07$ & & 23 & 11 & $0.68 \pm 0.08$ \\
\hline
\end{tabular}

compilations covering this energy range, show similar compatibility with experiment for Ebel's and EPDL cross sections, which is confirmed by the statistical comparison of the two categories in Table X.

4) Elam's and Veigele's Cross Sections: they cover energies between $100 \mathrm{eV}$ and $1 \mathrm{MeV}$. The efficiencies of the models applicable in this energy range are listed in Table XIV. The results of the categorical analysis in Table $\mathrm{X}$ show that the hypothesis of equivalent compatibility with experiment as for EPDL cross sections is rejected for Veigele's cross sections, while it is not rejected for Elam's.

5) Henke's Cross Sections: this model covers energies between $10 \mathrm{eV}$ and $30 \mathrm{keV}$. The efficiencies for the total cross section models applicable to these energies are listed in Table XV, which also reports values for photon energies above $150 \mathrm{eV}$. Henke's efficiency is lower; nevertheless the tests summarized in Table $\mathrm{X}$ do not reject the hypothesis of equivalent compatibility with experiment for Henke's and EPDL cross sections with 0.01 significance.

It is worth remarking that all models appear inadequate at reproducing experimental measurements in the lower energy
TABLE XVI

TOTAL CROSS SECTIONS: EFFICIENCY BETWEEN 10/150 EV AND $300 \mathrm{KEV}$

\begin{tabular}{lcccccccc}
\hline & \multicolumn{3}{c}{$\mathrm{E}_{\min }=10 \mathrm{eV}$} & & \multicolumn{3}{c}{$\mathrm{E}_{\min }=150 \mathrm{eV}$} \\
\cline { 2 - 4 } \cline { 7 - 9 } Model & Pass & Fail & Efficiency & & Pass & Fail & Efficiency \\
\hline BiggsG4 & 29 & 34 & $0.46 \pm 0.06$ & & 31 & 15 & $0.67 \pm 0.07$ \\
Biggs & 30 & 33 & $0.48 \pm 0.06$ & & 34 & 12 & $0.74 \pm 0.06$ \\
Chantler & 19 & 44 & $0.30 \pm 0.06$ & & 22 & 24 & $0.48 \pm 0.07$ \\
EPDL & 35 & 28 & $0.56 \pm 0.06$ & & 36 & 10 & $0.78 \pm 0.06$ \\
Penelope & 37 & 26 & $0.59 \pm 0.06$ & & 36 & 10 & $0.78 \pm 0.06$ \\
RTAB & 36 & 27 & $0.57 \pm 0.06$ & & 35 & 11 & $0.76 \pm 0.06$ \\
\hline
\end{tabular}

TABLE XVII

TOTAL CROSS SECTIONS: EFFICIENCY BETWEEN 1 KEV AND 500 KEV

\begin{tabular}{lccc}
\hline Model & Pass & Fail & Efficiency \\
\hline BiggsG4 & 27 & 8 & $0.77 \pm 0.07$ \\
Biggs & 27 & 8 & $0.77 \pm 0.07$ \\
Brennan & 22 & 13 & $0.63 \pm 0.08$ \\
Elam & 28 & 7 & $0.80 \pm 0.07$ \\
EPDL & 30 & 5 & $0.86 \pm 0.06$ \\
PHOTX & 28 & 7 & $0.80 \pm 0.07$ \\
Scofield & 28 & 7 & $0.80 \pm 0.07$ \\
Storm & 24 & 11 & $0.69 \pm 0.08$ \\
Veigele & 22 & 13 & $0.63 \pm 0.08$ \\
XCOM-DB & 26 & 9 & $0.74 \pm 0.07$ \\
XCOM & 29 & 6 & $0.83 \pm 0.06$ \\
\hline
\end{tabular}

range, while they perform better above $150 \mathrm{eV}$. Chantler's calculations remain largely incompatible with experiment even in the higher energy interval.

6) RTAB Total Cross Sections: this compilation covers energies between approximately $10 \mathrm{eV}$ and $300 \mathrm{keV}$. The efficiencies for the total cross section models applicable to these energies are listed in Table XVI, which also reports values for photon energies above $150 \mathrm{eV}$. The efficiency for RTAB cross sections is similar to that obtained with EPDL; the results of the statistical analysis of the related contingency table are consistent with this observation.

It is worth remarking that all models appear inadequate at reproducing experimental measurements in the lower energy range, while they perform better above $150 \mathrm{eV}$. Chantler's calculations remain largely incompatible with experiment even in the higher energy interval.

7) XCOM-DB Cross Sections: this compilation, encompassed in the DABAX database, concerns energies between $1 \mathrm{keV}$ and $500 \mathrm{keV}$. The efficiencies of the applicable total cross section models are listed in Table XVII. Neither the tests on contingency tables concerning XCOM-DB and EPDL (reported in Table X), nor those concerning XCOM-DB and the standard XCOM tabulation reject the hypothesis of equivalent compatibility with experiment.

The different efficiency for cross sections based on XCOMDB and standard XCOM tabulations could derive from a different energy grid of the two tabulations, which affects the cross section values calculated by interpolation, or from a different version of XCOM used as a basis for XCOM-DB. The $\mathrm{XCOM}$ version used for creating the XCOM-DB tabulations could not be retrieved in the DABAX documentation.

The quantitative validation analysis documented here shows that none of the specialized cross section models provides better 
TABLE XVIII

EFFICIENCY OF TOTAL CROSS SECTION MODELS IN THE LOW ENERGY RANGE

\begin{tabular}{lccccccc}
\hline & \multicolumn{3}{c}{$10-100 \mathrm{eV}$} & & \multicolumn{3}{c}{$10-150 \mathrm{eV}$} \\
\cline { 2 - 4 } \cline { 7 - 8 } Model & Pass & Fail & Efficiency & & Pass & Fail & Efficiency \\
\hline BiggsG4 & 9 & 22 & $0.29 \pm 0.08$ & & 7 & 28 & $0.20 \pm 0.07$ \\
Biggs & 5 & 26 & $0.16 \pm 0.07$ & & 8 & 27 & $0.23 \pm 0.07$ \\
Chantler & 4 & 27 & $0.13 \pm 0.08$ & & 5 & 30 & $0.14 \pm 0.06$ \\
EPDL & 8 & 23 & $0.26 \pm 0.06$ & & 10 & 25 & $0.29 \pm 0.07$ \\
Henke & 9 & 22 & $0.29 \pm 0.08$ & & 11 & 24 & $0.31 \pm 0.08$ \\
Penelope & 10 & 21 & $0.32 \pm 0.08$ & & 12 & 23 & $0.34 \pm 0.08$ \\
RTAB & 10 & 21 & $0.32 \pm 0.08$ & & 12 & 23 & $0.34 \pm 0.08$ \\
\hline
\end{tabular}

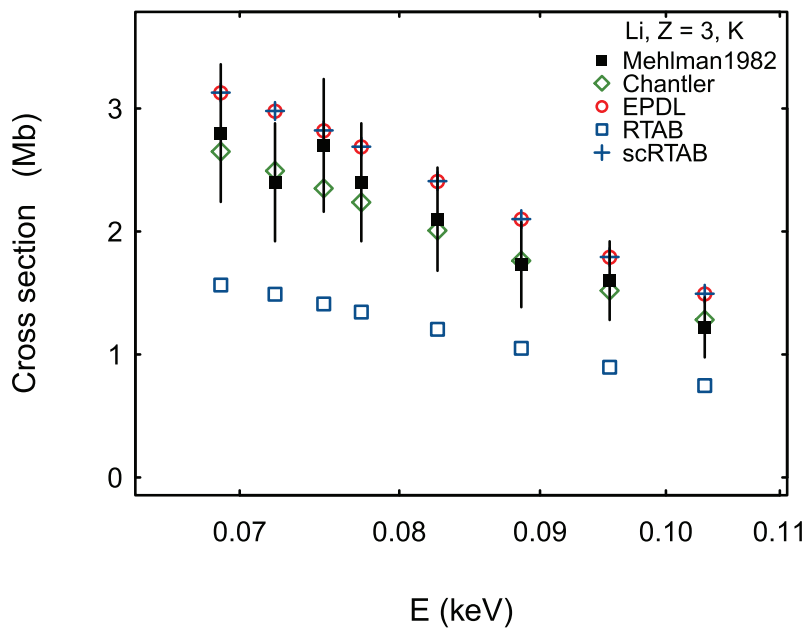

Fig. 33. Cross section for the photoionization of the $\mathrm{K}$ shell of lithium as a function of photon energy.

accuracy than EPDL tabulations: some of them exhibit significantly worse compatibility with experiment, while others are at most statistically equivalent to EPDL at reproducing experimental measurements. Therefore their use in their specific range of applicability at the place of EPDL would not improve the accuracy of general purpose Monte Carlo codes.

\section{Evaluation of Total Cross Sections at Low Energy}

The results documented in the previous sections hint that neither the models applicable to an extended energy range nor those covering specific energies appear capable of reproducing cross sections measurements at low energies.

$\chi^{2}$ tests performed over a data sample with energies between $10 \mathrm{eV}$ and $150 \mathrm{eV}$ quantify the capability of all models applicable in that energy range to reproduce experimental data. The resulting efficiencies are documented in Table XVIII; they confirm that none of the cross section compilation is suitable for accurate simulation of the photoelectric effect in this energy range.

\section{ViII. Results of Partial Cross Section Validation}

Figs. 33 to 51 illustrate some examples of calculated and experimental cross sections for inner and outer shell photoionization.

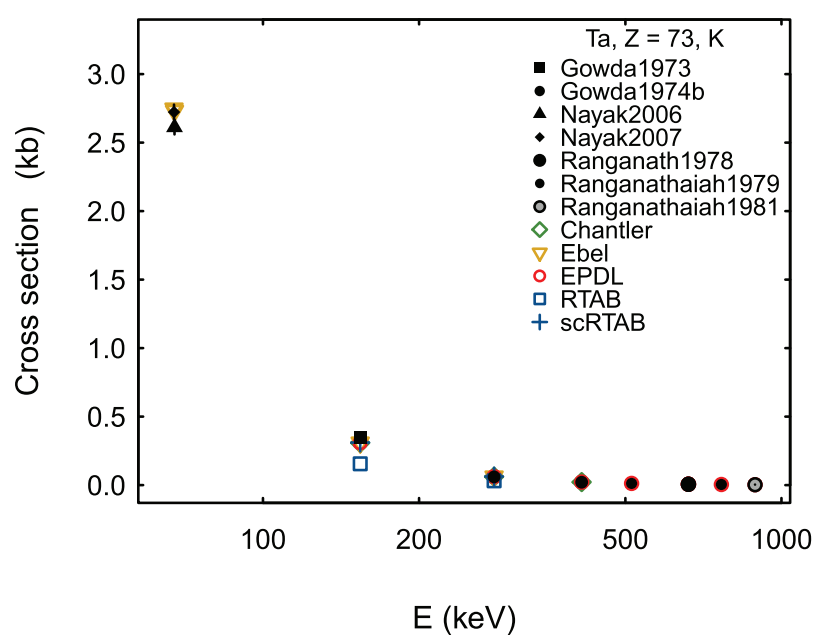

Fig. 34. Cross section for the photoionization of the $\mathrm{K}$ shell of tantalum as a function of photon energy.

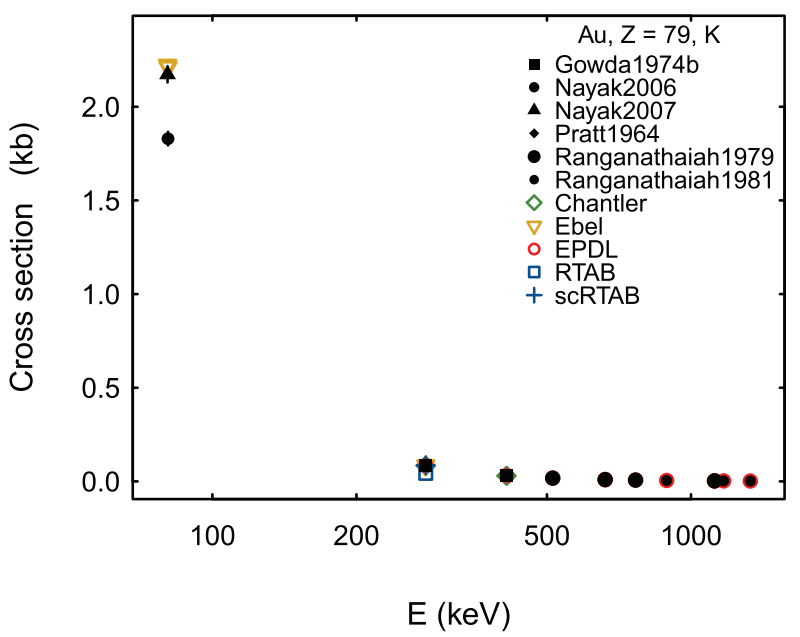

Fig. 35. Cross section for the photoionization of the $\mathrm{K}$ shell of gold as a function of photon energy.

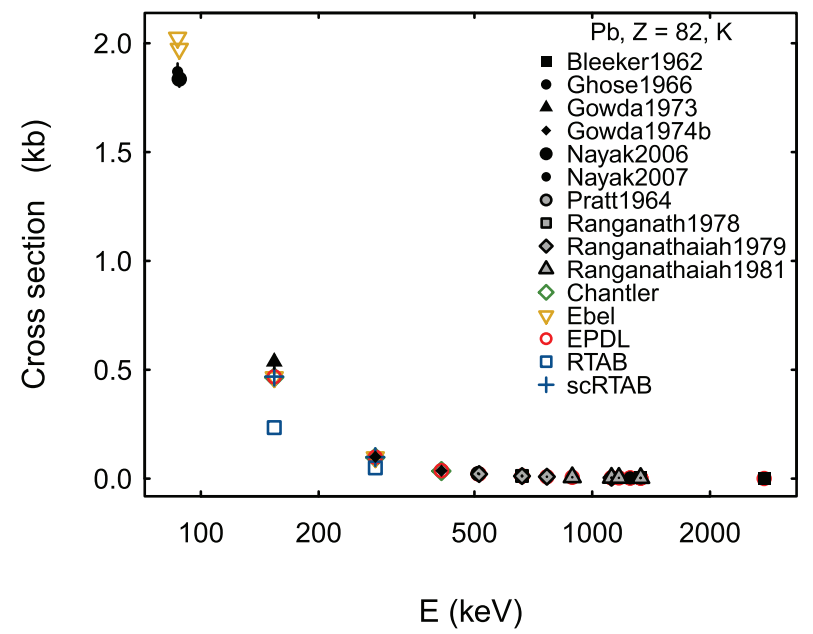

Fig. 36. Cross section for the photoionization of the $\mathrm{K}$ shell of lead as a function of photon energy.

A systematic discrepancy of RTAB shell cross sections with respect to experimental data is observed, which hints at a missing multiplicative factor in the tabulated values. RTAB cross 


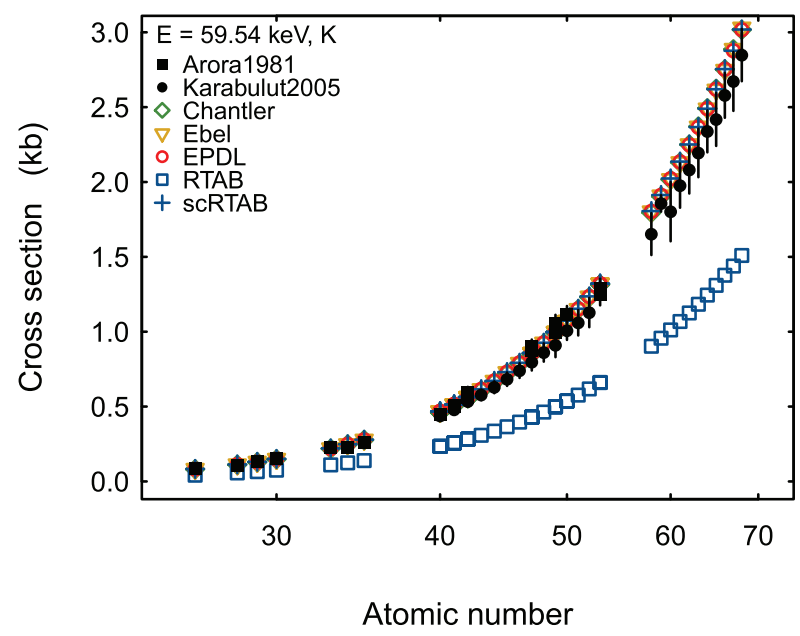

Fig. 37. Cross section for the photoionization of the $\mathrm{K}$ shell at $59.54 \mathrm{keV}$ as a function of the atomic number.

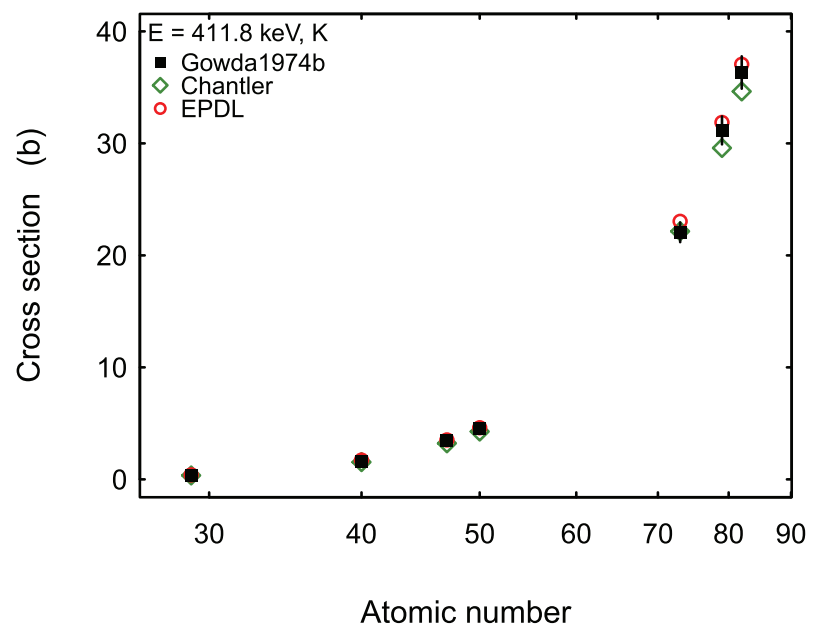

Fig. 38. Cross section for the photoionization of the $\mathrm{K}$ shell at $411.8 \mathrm{keV}$ as a function of the atomic number.

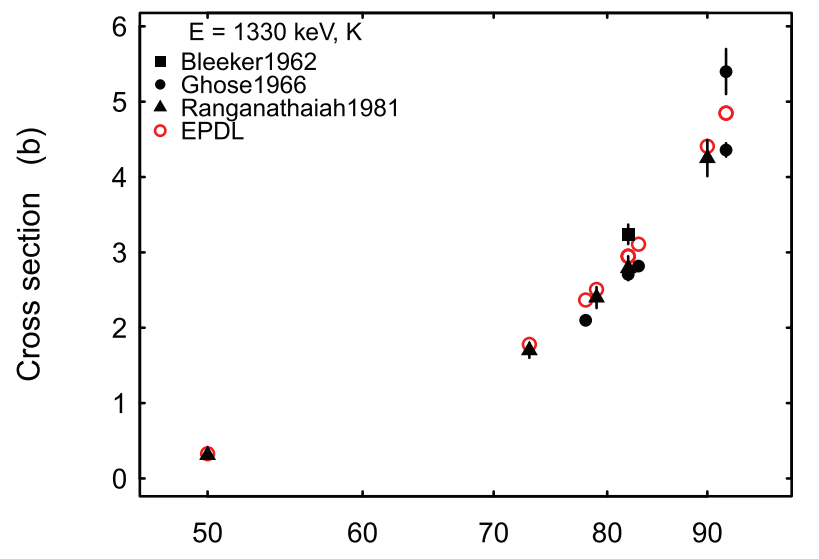

Atomic number

Fig. 39. Cross section for the photoionization of the $\mathrm{K}$ shell at $1.33 \mathrm{MeV}$ as a function of the atomic number.

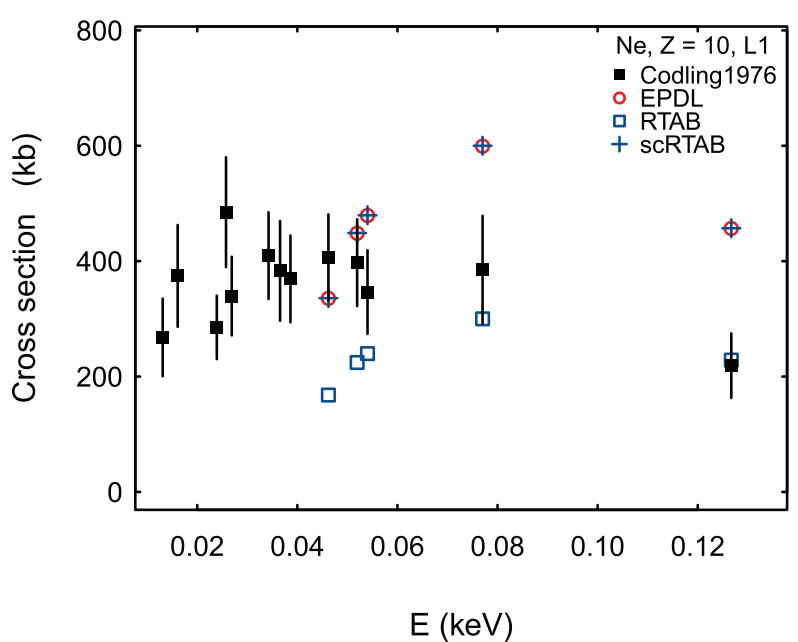

Fig. 40. Cross section for the photoionization of the $\mathrm{L}_{1}$ subshell of neon as a function of photon energy.

sections scaled by the presumed missing factor are identified in figures and in the following tables as "scRTAB".

\section{A. K Shell}

All but one of the experimental data sets concern energies above $1 \mathrm{keV}$.

Only the compilations based on Scofield's 1973 nonrelativistic calculations cover the whole energy range of the experimental data sample above $1 \mathrm{keV}$; their efficiencies are listed in Table XIX. The lower efficiency obtained with PHOTX is related to the characteristics of the energy grid over which cross sections are tabulated.

Chantler's, EPDL and scRTAB models are applicable to the single set of measurements below $1 \mathrm{keV}$ retrieved from the literature; according to the outcome of the $\chi^{2}$ test, their calculations are compatible with the experimental data set with 0.01 significance.

The capabilities of Chantler's, Ebel's and scRTAB models, which are limited to a specific energy range, are evaluated by restricting the $\chi^{2}$ test of comparison with experiment to the data sample consistent with their coverage. The resulting efficiencies, calculated within $1 \mathrm{keV}$ and $300 \mathrm{keV}$, which correspond to the coverage of Ebel's model, are reported in Table XX. Since the experimental data sample includes no additional measurements between $300 \mathrm{keV}$ and $433 \mathrm{keV}$, which is the highest energy tabulated by Chantler's calculations, the results in Table XX express the outcome of the validation test for this model too.

Ebel's parameterization appears more efficient than EPDL tabulations at reproducing experimental $\mathrm{K}$ shell cross sections in the energy range between 1 and $300 \mathrm{keV}$; nevertheless, the hypothesis of equivalent compatibility with experiment for these $\mathrm{K}$ shell cross section models is not rejected by any of the tests applied to the analysis of the associated contingency table with 0.01 significance. The failures of EPDL-based cross sections at reproducing experimental data mostly concern test cases close to absorption edges. Issues about the accuracy of related calculations of atomic binding energies 


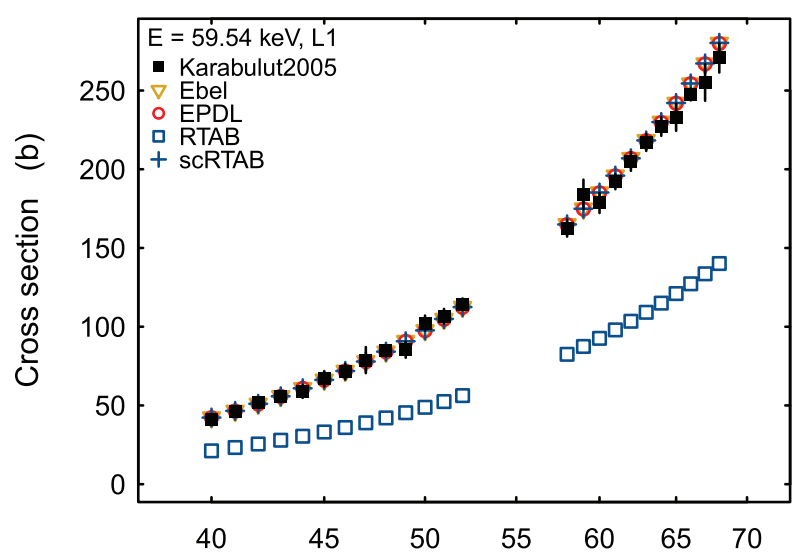

Atomic number

(a) $\mathrm{L}_{1}$ subshell

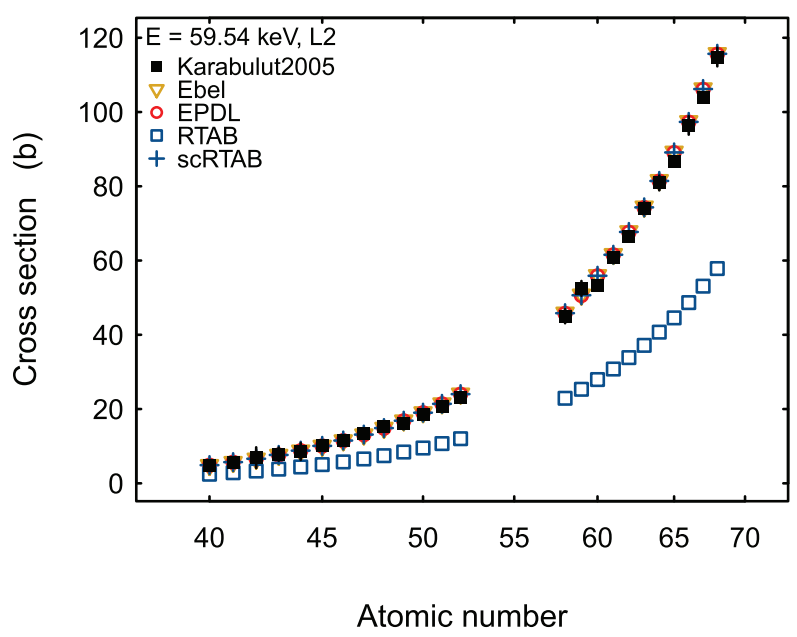

(b) $\mathrm{L}_{2}$ subshell

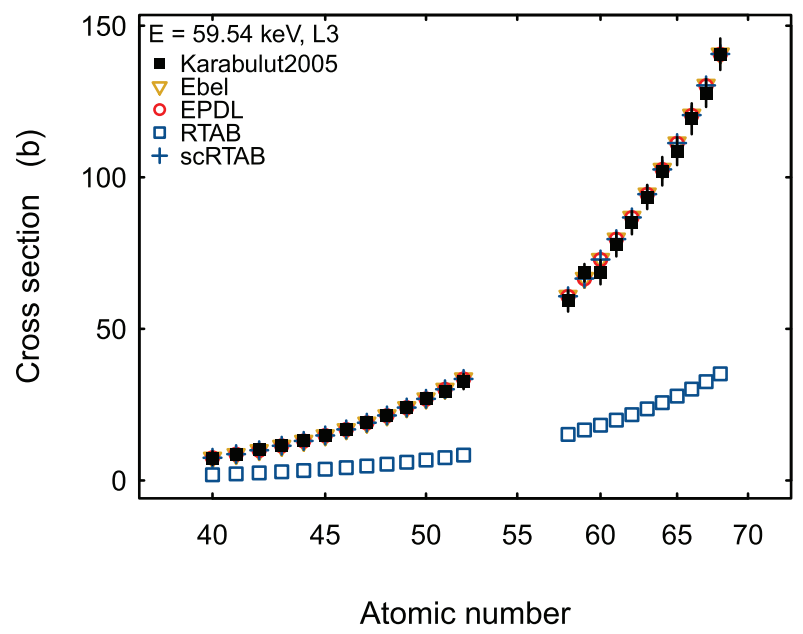

(c) $\mathrm{L}_{3}$ subshell

Fig. 41. Cross section for the photoionization of the $\mathrm{L}$ shell at $59.54 \mathrm{keV}$ as a function of the atomic number. (a) $\mathrm{L}_{1}$ subshell (b) $\mathrm{L}_{2}$ subshell (c) $\mathrm{L}_{3}$ subshell.

collected in EADL (Evaluated Atomic Data Library) [267] were documented in [22]; as discussed in Section VI-B, experimental measurements in these regions are delicate, as

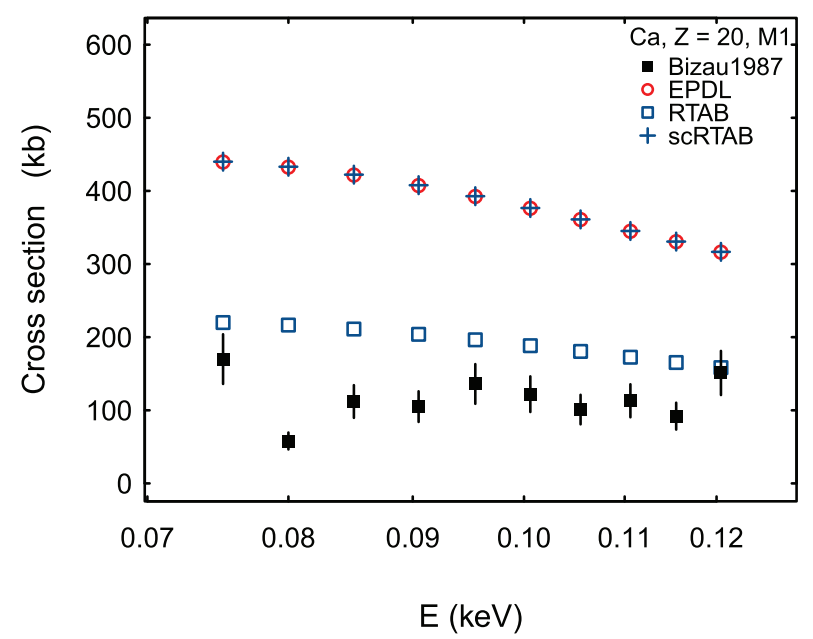

(a) $\operatorname{Argon}(\mathrm{Z}=18)$

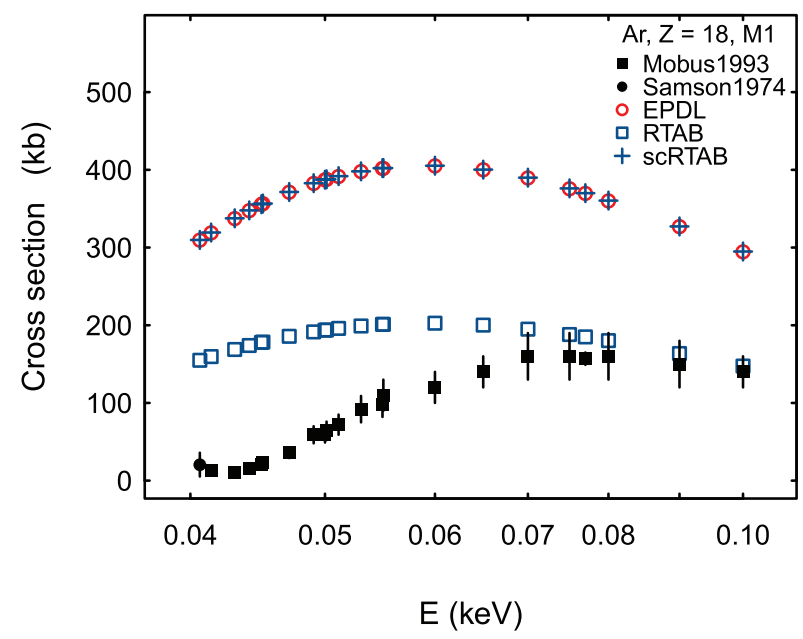

(b) Calcium $(\mathrm{Z}=20)$

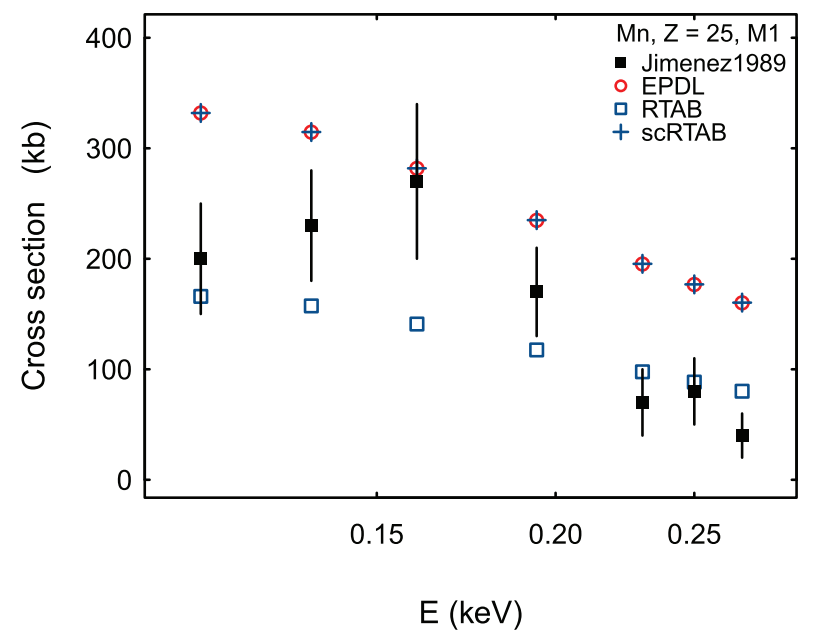

(c) Manganese $(\mathrm{Z}=25)$

Fig. 42. Cross section for the photoionization of the $M_{1}$ subshell of argon, calcium and manganese as a function of energy. (a) Argon $(Z=18)$ (b) Calcium $(Z=20)$ (c) Manganese $(Z=25)$.

realistic estimates of experimental uncertainties are prone to be affected by systematic effects deriving from the experimental environment. 


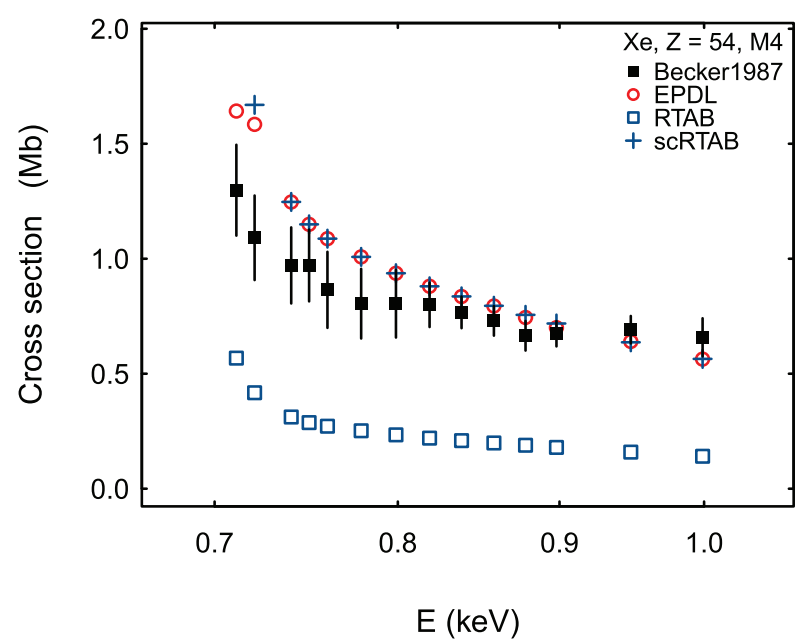

Fig. 43. Cross section for the photoionization of the $\mathrm{M}_{4}$ subshell of xenon as a function of photon energy.

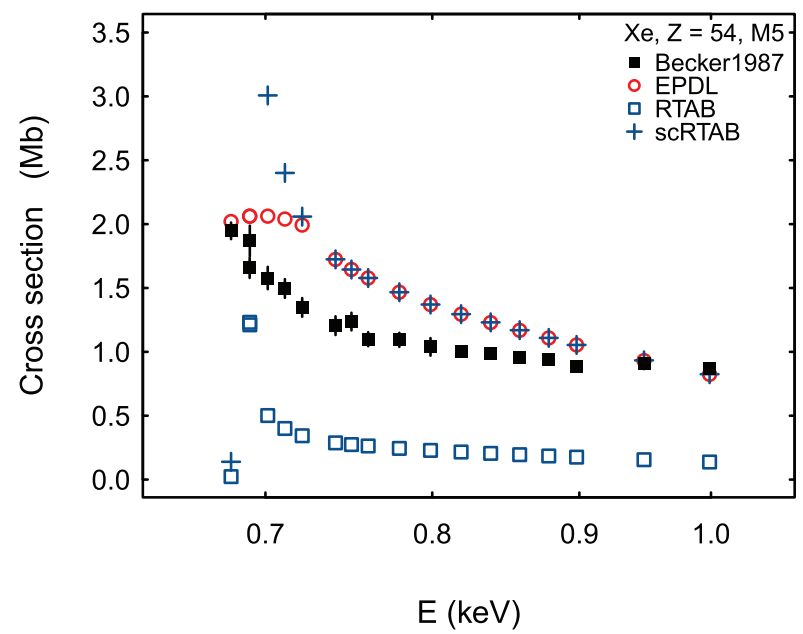

Fig. 44. Cross section for the photoionization of the $\mathrm{M}_{5}$ subshell of xenon as a function of photon energy.

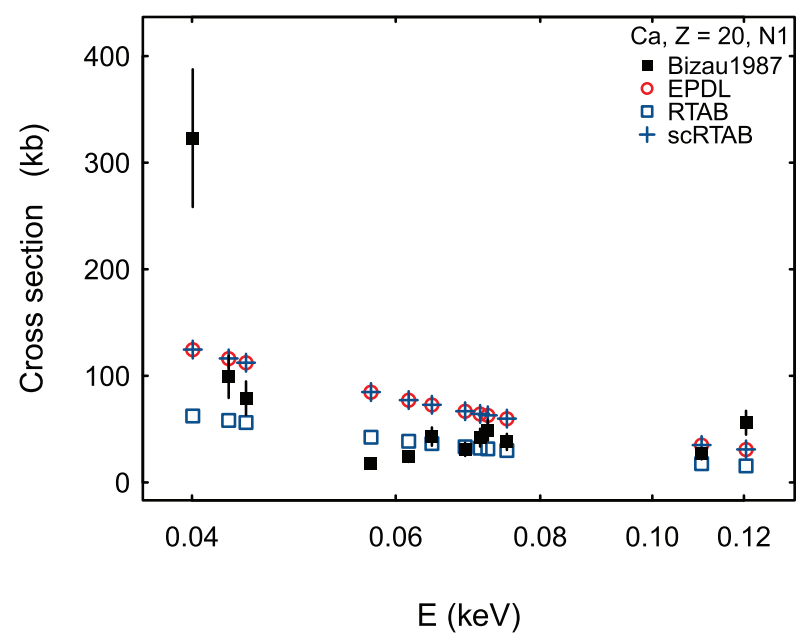

Fig. 45. Cross section for the photoionization of the $\mathrm{N}_{1}$ subshell of calcium as a function of photon energy.

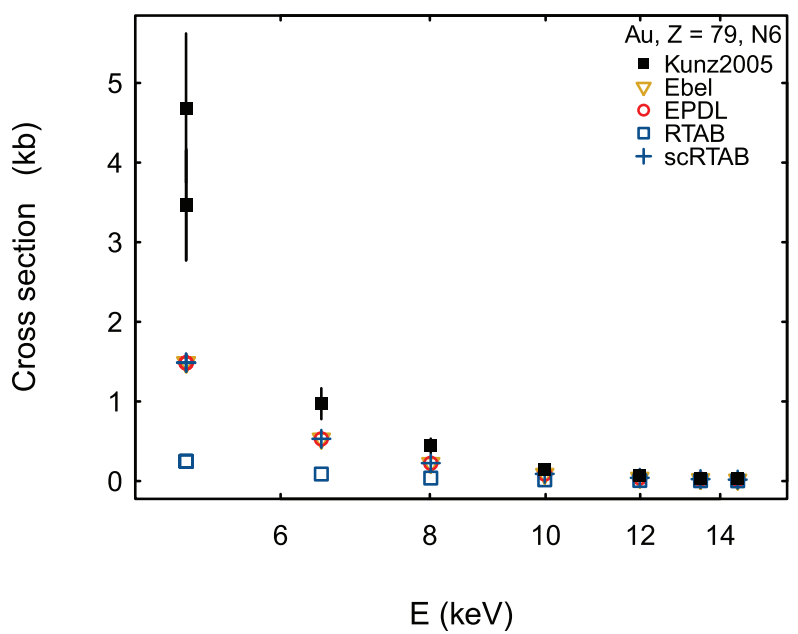

Fig. 46. Cross section for the photoionization of the $\mathrm{N}_{6}$ subshell of gold as a function of photon energy.

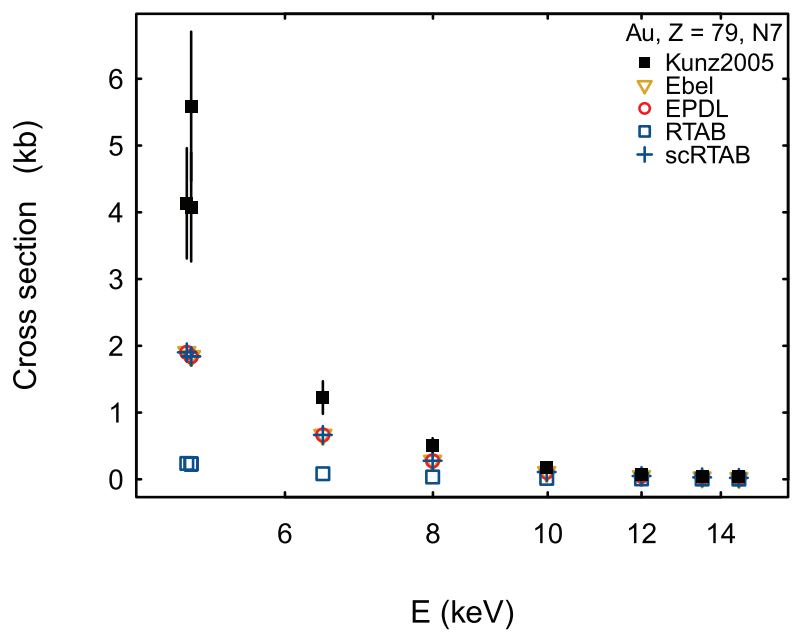

Fig. 47. Cross section for the photoionization of the $\mathrm{N}_{7}$ subshell of gold as a function of photon energy.

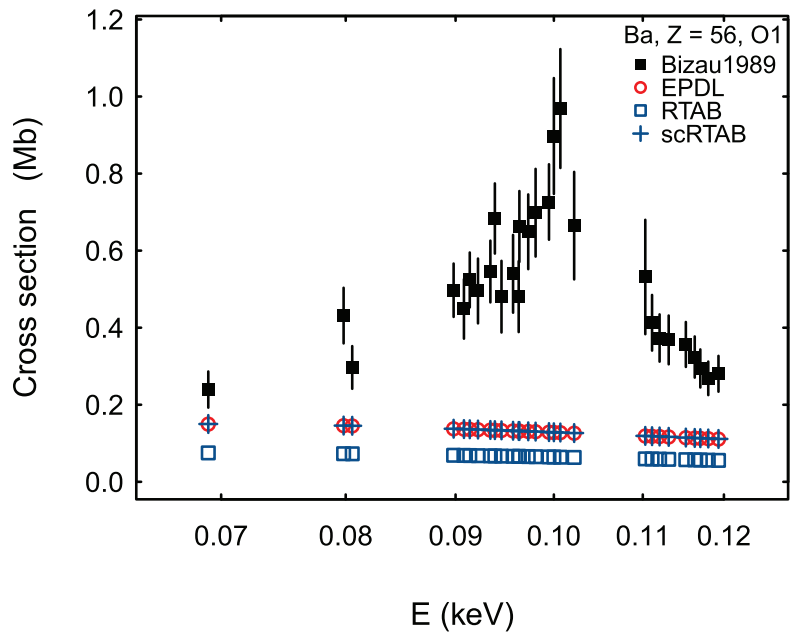

Fig. 48. Cross section for the photoionization of the $\mathrm{O}_{1}$ subshell of barium as a function of photon energy. 


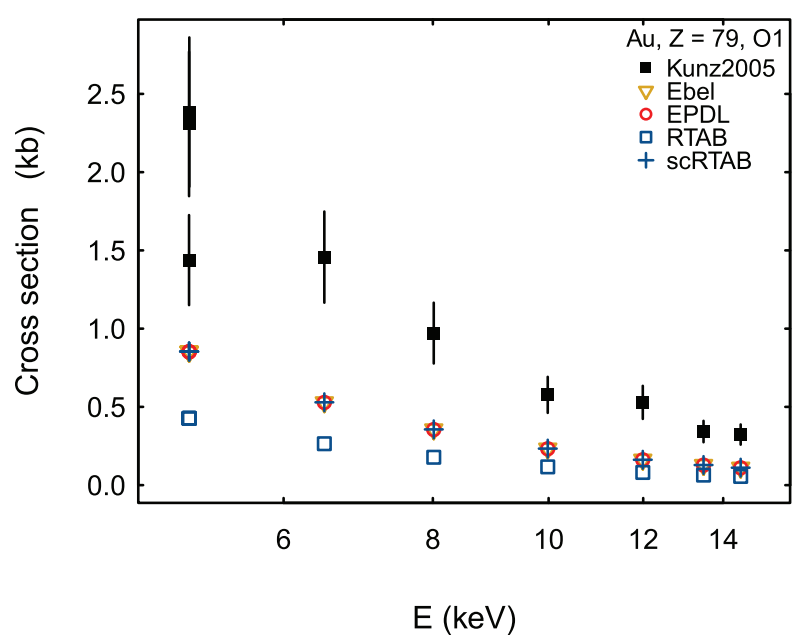

Fig. 49. Cross section for the photoionization of the $\mathrm{O}_{1}$ subshell of gold as a function of photon energy.

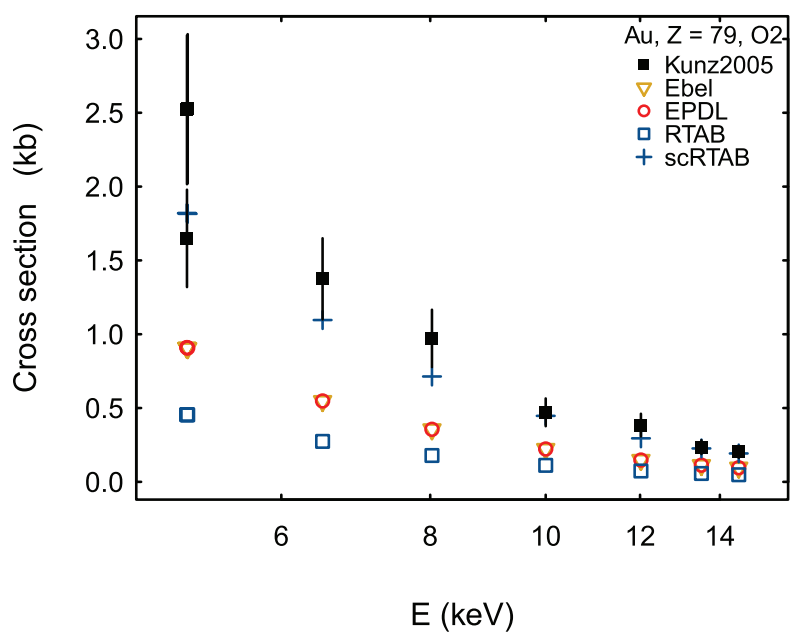

Fig. 50. Cross section for the photoionization of the $\mathrm{O}_{2}$ subshell of gold as a function of photon energy.

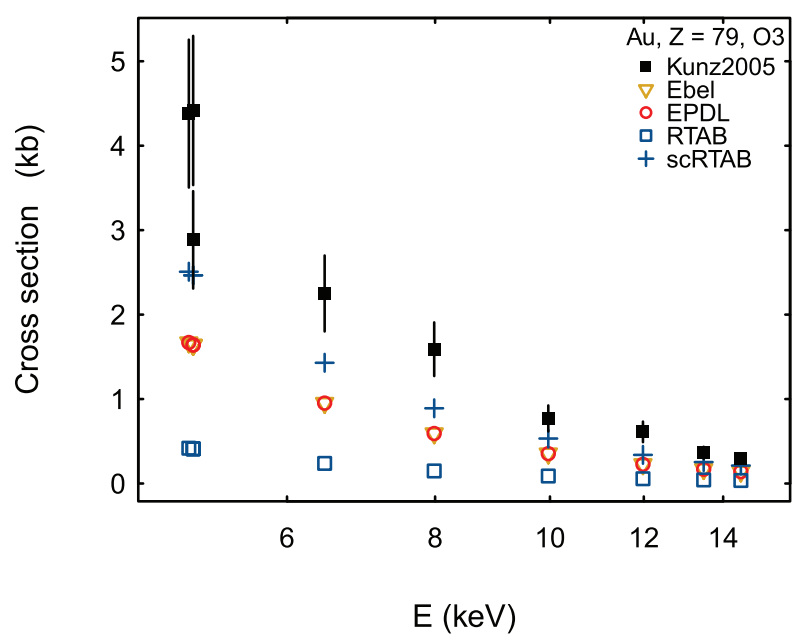

Fig. 51. Cross section for the photoionization of the $\mathrm{O}_{3}$ subshell of gold as a function of photon energy.
TABLE XIX

K SHELL CROSS SECTION EFFICIENCY OF MODELS BASED ON SCOFIELD 1973 NON-RELATIVISTIC CALCULATIONS

\begin{tabular}{lccc}
\hline Model & Pass & Fail & Efficiency \\
\hline EPDL & 62 & 17 & $0.78 \pm 0.05$ \\
Penelope & 62 & 17 & $0.78 \pm 0.05$ \\
PHOTX & 52 & 27 & $0.66 \pm 0.05$ \\
Scofield & 62 & 17 & $0.78 \pm 0.05$ \\
\hline
\end{tabular}

TABLE XX

EFFICIENCY OF K SHELL CROSS SECTION MODELS FOR ENERGIES BETWEEN $1 \mathrm{KEV}$ AND $300 \mathrm{KEV}$

\begin{tabular}{lccc}
\hline Model & Pass & Fail & Efficiency \\
\hline Chantler & 45 & 13 & $0.78 \pm 0.05$ \\
Ebel & 50 & 8 & $0.86 \pm 0.05$ \\
EPDL & 45 & 13 & $0.78 \pm 0.05$ \\
Penelope & 45 & 13 & $0.78 \pm 0.05$ \\
PHOTX & 47 & 11 & $0.81 \pm 0.05$ \\
scRTAB & 45 & 13 & $0.78 \pm 0.05$ \\
Scofield & 45 & 13 & $0.78 \pm 0.05$ \\
\hline
\end{tabular}

TABLE XXI

EFFICIENCY OF L SHELL CROSS SECTION MODELS ABOVE $1 \mathrm{KEV}$

\begin{tabular}{clccc}
\hline Subshell & Model & Pass & Fail & Efficiency \\
\hline \multirow{6}{*}{ L $_{1}$} & Ebel & 24 & 0 & $1.00-0.04$ \\
& EPDL & 24 & 0 & $1.00-0.04$ \\
& Penelope & 24 & 0 & $1.00-0.04$ \\
& PHOTX & 24 & 0 & $1.00-0.04$ \\
& scRTAB & 24 & 0 & $1.00-0.04$ \\
& Scofield & 24 & 0 & $1.00-0.04$ \\
\hline \multirow{6}{*}{ L $_{2}$} & Ebel & 23 & 1 & $0.96 \pm 0.04$ \\
& EPDL & 23 & 1 & $0.96 \pm 0.04$ \\
& Penelope & 23 & 1 & $0.96 \pm 0.04$ \\
& PHOTX & 23 & 1 & $0.96 \pm 0.04$ \\
& scRTAB & 23 & 1 & $0.96 \pm 0.04$ \\
& Scofield & 23 & 1 & $0.96 \pm 0.04$ \\
\hline \multirow{6}{*}{ L $_{3}$} & Ebel & 27 & 0 & $1.00-0.03$ \\
& EPDL & 27 & 0 & $1.00-0.03$ \\
& Penelope & 27 & 0 & $1.00-0.03$ \\
& PHOTX & 27 & 0 & $1.00-0.03$ \\
& scRTAB & 27 & 0 & $1.00-0.03$ \\
& Scofield & 27 & 0 & $1.00-0.03$ \\
\hline \multirow{6}{*}{} & & & &
\end{tabular}

\section{B. L Shell}

All L subshell measurements included in the experimental data sample concern energies between $1 \mathrm{keV}$ and $300 \mathrm{keV}$, with the exception of one set of $\mathrm{L}_{1}$ cross section data, which encompasses lower energy measurements.

Efficiencies above $1 \mathrm{keV}$ are reported in Table XXI for all models dealing with $\mathrm{L}$ subshells. All cross section models are equally capable of reproducing the measurements in the experimental data sample.

The hypothesis of compatibility with the single experimental data set including measurements below $1 \mathrm{keV}$ is rejected for all models subject to evaluation. Nevertheless, no conclusions regarding the capabilities of the cross section models at lower energies can be inferred from such a limited test; the failure over a single experimental data set could be due either to deficiency of the models or to systematic effects affecting the measurements, which cannot be investigated in terms of consistency with other, independent experimental measurements. 


\section{Outer Shells}

The cross sections for outer shells appear incompatible with experiment; the $\chi^{2}$ test rejects the hypothesis of compatibility between calculated and experimental cross sections in most test cases. Nevertheless, caution should be exercised in drawing general conclusions from these results, as the experimental data sample for the validation of outer shells is small and for a given test case the available data often originate from a single experimental source. This test scenario is prone to be affected by systematic effects, which would hinder the reliability of the outcome of the statistical analysis.

Firm conclusions about the accuracy of cross section calculations for outer shells would require more extensive experimental data samples.

\section{Qualitative Evaluation of Photoelectron ANGULAR DisTRIBUTION}

A preliminary appraisal of photoelectron angular distribution models has been performed, limited to the different options implemented in Geant4 and GEANT 3. Inconsistencies in the analytical formula of the latter appearing in the software documentation have been corrected in the implementation used for this evaluation.

Reports of direct measurements of photoelectron angular distribution are scarce in the literature; the narrow scope of the experimental data sample we could retrieve from the literature and the lack of adequate detail on the characteristics of the reported measurements prevent a meaningful statistical study for the validation of the models used in general purpose Monte Carlo codes.

Figs. 52 and 53 show qualitative comparisons of photoelectron angular distribution models implemented in Geant 4 and GEANT 3 with experimental data; discrepancies are visible, both between calculations and experimental data and between the outcome of different model implementations, which suggest that further quantitative investigation would be useful. In the scenarios corresponding to the limited experimental sample retrieved from the literature, Geant 4 angular distribution models exhibit similar behaviour; the corrected GEANT 3 model appears in some cases different from the others and qualitatively closer to measurements. Nevertheless, no conclusions can be drawn from such a narrow, qualitative test.

An indirect investigation of photoelectric differential cross section models could be pursued through testing the so-called "asymmetry parameter" [5]; due to its complexity, this study should be considered as the subject of a dedicated paper.

\section{COnClusion}

An extensive set of cross section models for the simulation of photoionization has been quantitatively evaluated regarding their accuracy at reproducing experimental measurements. Since the validation analysis documented in this paper concerns cross section data libraries and parameterization methods, which are (and can be) used by any Monte Carlo codes, rather than specific implementations of the photoelectric effect, its

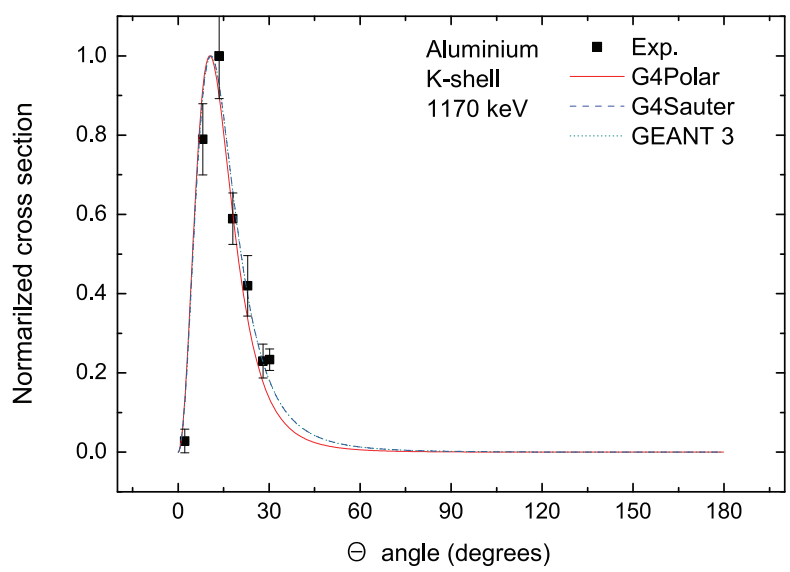

Fig. 52. Photoelectron angular distribution for aluminium, $\mathrm{K}$ shell, at $1.17 \mathrm{MeV}$.

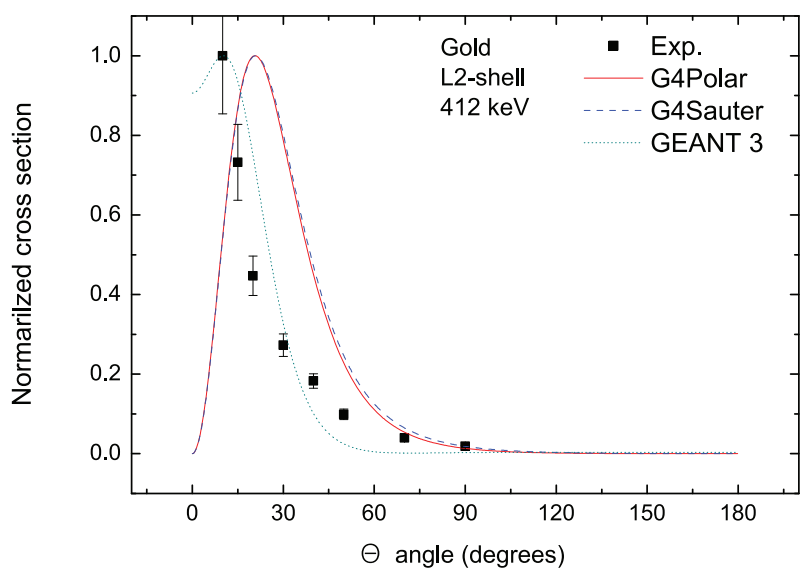

Fig. 53. Photoelectron angular distribution for gold, $\mathrm{L}_{2}$ subshell, at $412 \mathrm{keV}$.

results, and the recommendations which derive from them, are of general relevance for the simulation of the photoelectrict effect.

Statistical tests against a large sample of total and partial cross section measurements demonstrate that tabulations based on Scofield's 1973 non-relativistic calculations, which cover an extended range of energies starting from a few electronvolts, achieve the best overall capability to reproduce experiment among the models subject to test. Small differences in the results of goodness-of-fit tests are observed across different compilations (EPDL, Penelope database, PHOTX, XCOM) based on the same theoretical calculation method; they appear related to the granularity of the tabulations.

Some discrepancies with experiment of EPDL-based K shell cross sections are observed close to absorption edges, which could be related to intrinsic limitations of the calculations, also highlighted in the evaluation of related calculations of atomic binding energies; nevertheless, the possibility of systematic effects in these delicate measurements suggests caution in drawing conclusions about the reliability of EPDL in the proximity of absorption edges. 
No other photoelectric cross section calculation methods, among those considered in this study, demonstrate statistically better accuracy than EPDL tabulations: neither theoretical calculations, including relativistic ones, nor empirical or theory-driven analytical parameterizations. The validation tests documented in this paper demonstrate that the compatibility with experiment of some of them is significantly worse than that of EPDL, while it is statistically equivalent for others, although restricted to the energy range they cover. Only K-shell cross sections based on Ebel's parameterization produce more accurate results than EPDL in some test cases close to absorption edges, although the difference in compatibility with experiment with respect to EPDL is not statistically significant.

Special attention has been devoted to the evaluation of total cross sections based on Biggs and Lighthill's parameterization, which is extensively used in Geant 4 . While the original set of parameterization coefficients produces cross sections that are statistically equivalent to EPDL regarding compatibility with experiment, the modified coefficients implemented in Geant 4 significantly worsen the resulting cross section compatibility with experimental measurements above a few tens of electronvolt. The original and modified parameterization produce statistically equivalent compatibility with experiment below a few tens of electronvolt; both parameterizations, as well as other total cross section models, exhibit low efficiency in that energy range. As a result of this validation test, it is recommended that future versions of Geant 4 revert to using the original coefficients of Biggs and Lighthill parameterization above a few tenso of electronvolt, unless a new, more accurate parameterization is developed and quantitatively demonstrated to improve compatibility with experiment over the original one. The suggestion to implement this modification in Geant 4 version 10.2, which is in preparation at the time of writing this paper, has been conveyed to the maintainers of the related Geant 4 code. Given that EPDL photoelectric cross sections are already used in other Geant4 models [93], the burden of maintaining alternative cross sections based on Biggs-Lighthill's parameterization should be considered as well, as this validation test has demonstrated that even the original parameterization is not statistically superior to EPDL in reproducing experimental data.

The validation tests show that in the low energy end cross sections based on Scofield's non-relativistic calculations produce results consistent with experiment with 0.01 significance down to approximately $150 \mathrm{eV}$. Nevertheless, the limited representativity of the experimental data sample, which only includes noble gases and hydrogen, suggests caution at extrapolating this result to experimental scenarios involving solid targets.

No model, among those considered in this validation study, is able to reproduce experimental data consistently below approximately $150 \mathrm{eV}$. Their failure at low energies reflects the inadequacy of theoretical calculations in independent particle approximation and of the assumption of isolated atoms adopted in general purpose Monte Carlo codes, in conditions where effects related to the structure of the interacting material should be taken into account.

The quantitative results of the validation tests documented in this paper allow Monte Carlo code developers and experimental users to base the selection of physics models in their simulations on sound scientific grounds.

The authors are grateful to Lynn Kissel for providing a copy of the RTAB database. The authors thank Sergio Bertolucci for support, Vladimir Grichine and Georg Weidenspointner for valuable discussions, and Anita Hollier for proofreading the manuscript. The CERN Library has provided helpful assistance and essential reference material for this study.

\section{REFERENCES}

[1] R. H. Pratt, A. Ron, and H. K. Tseng, "Atomic photoeffect above 10 keV," Rev. Mod. Phys., vol. 45, pp. 273-325, 1973.

[2] R. H. Pratt, A. Ron, and H. K. Tseng, "Erratum: Atomic photoeffect above $10 \mathrm{keV}, "$ Rev. Mod. Phys., vol. 45, pp. 663-664, 1973.

[3] J. A. R. Samson, "Photoionization of atoms and molecules," Phys. Rep., vol. 28, no. 4, pp. 303-354, 1976.

[4] H. P. Kelly, "Review of our present understanding of the photoionization process for atoms," Proc. AIP Conf., 1990, vol. 215, pp. 292-311.

[5] A. F. Starace, "Atomic photoionization," Fundamental Processes in Energetic Atomic Collisions, H. O. Lutz, J. S. Briggs, H. Kleinpoppin, Eds. New York, NY, USA: Plenum, 1983.

[6] A. F. Starace, "Photoionization of atoms," Atomic, Molecular and Optical Physics Handbook, Berlin, Germany: Springer, 2006, pp. 379390.

[7] M. Y. Amusia, Atomic Photoeffect, New York, NY, USA: Plenum, 1990.

[8] J. Berkowitz, Atomic and Molecular Photoabsorption, London, U.K. Academic, 2002.

[9] J. H. Hubbell, "Review and history of photon cross section calculations," Phys. Med. Biol., vol. 51, pp. R245-R262, 2006.

[10] J. J. De Marco et al., "An analysis of MCNP cross-sections and tally methods for low-energy photon emitters," Phys. Med. Biol., vol. 47, pp. 1321-1332, 2002.

[11] K. Amako et al., "Comparison of geant4 electromagnetic physics models against the NIST reference data," IEEE Trans. Nucl. Sci., vol. 52, no. 4, pp. 910-918, Aug. 2005.

[12] H. Zaidi, "Comparative evaluation of photon cross section libraries for materials of interest in PET Monte Carlo simulation," IEEE Trans. Nucl. Sci., vol. 47, no. 6, pp. 2722-2735, Dec. 2000.

[13] U. Chica, M. Anguiano, and A. M. Lallena, "Benchmark of PENELOPE for low and medium energy X-rays," Phys. Med., vol. 25, no. 2, pp. 5157, 2009.

[14] H. Hirayama, Y. Namito, A. F. Bielajew, S. J. Wilderman, and W. R. Nelson, The EGS5 Code System, Stanford, CA, USA: SLAC-R-730 Rep., 2006.

[15] T. G. Trucano, L. P. Swiler, T. Igusa, W. L. Oberkampf, and M. Pilch, "Calibration, validation, and sensitivity analysis: Whats what," Reliab. Eng. Syst. Safety, vol. 91, no. 10-11, pp. 1331-1357, 2006.

[16] G. A. P. Cirrone, G. Cuttone, F. Di Rosa, L. Pandola, F. Romano, and Q. Zhang, "Validation of the geant4 electromagnetic photon crosssections for elements and compounds," Nucl. Instrum. Methods Phys. Res. A, vol. 618, pp. 315-322, 2010.

[17] S. Agostinelli et al., "Geant4 - a simulation toolkit," Nucl. Instrum. Methods Phys. Res. A, vol. 506, no. 3, pp. 250-303, 2003.

[18] J. Allison et al., "Geant4 developments and applications," IEEE Trans. Nucl. Sci., vol. 53, no. 1, pp. 270-278, Feb. 2006.

[19] S. Guatelli, A. Mantero, B. Mascialino, P. Nieminen, and M. G. Pia, "Geant4 atomic relaxation," IEEE Trans. Nucl. Sci., vol. 54, no. 3, pp. 585-593, Jun. 2007.

[20] S. Guatelli et al., "Validation of geant4 atomic relaxation against the NIST physical reference data," IEEE Trans. Nucl. Sci., vol. 54, no. 3, pp. 594-603, Jun. 2007.

[21] M. G. Pia, P. Saracco, and M. Sudhakar, "Validation of K and L shell radiative transition probability calculations," IEEE Trans. Nucl. Sci., vol. 56, no. 6, pp. 3650-3661, Dec. 2009.

[22] M. G. Pia et al., "Evaluation of atomic electron binding energies for Monte Carlo particle transport," IEEE Trans. Nucl. Sci., vol. 58, no. 6, pp. 3246-3268, Dec. 2011.

[23] H. Hirayama and Y. Namito, Implementation of a general treatment of photoelectric-related phenomena for compounds or mixtures in EGS4, Tsukuba, Japan: KEK Internal 2000-3 Rep., 2000.

[24] H. Hirayama and Y. Namito, Implementation of a General Treatment of Photoelectric-Related Phenomena for Compounds or Mixtures in EGS4 (Revised Version), Tsukuba, Japan: KEK Internal 2004-6 Rep., 2004. 
[25] R. H. Pratt, "Atomic photoelectric effect at high energies," Phys. Rev., vol. 117, pp. 1017-1028, 1960 .

[26] R. H. Pratt, "K-shell photoelectric cross sections from $200 \mathrm{keV}$ to 2 MeV,” Phys. Rev., vol. 134, pp. A898-A915, 1964.

[27] G. Rakavy and A. Ron, "Atomic photoeffect in the range $\mathrm{E}_{\gamma}=1-$ 2000 keV," Phys. Rev., vol. 159, pp. 50-56, 1960.

[28] R. D. Schmickley and R. Pratt, "K-, L-, and M-shell atomic photoeffect for screened-potential models," Phys. Rev., vol. 164, pp. 104-116, 1967.

[29] J. H. Scofield, Theoretical photoionization cross sections from 1 to $1500 \mathrm{keV}$, Lawrence Livermore Laboratory Rep. UCRL-51326, 1973.

[30] C. T. Chantler, "Theoretical form factor, attenuation and scattering tabulation for $\mathrm{Z}=1-92$ from $\mathrm{E}=1-10 \mathrm{eV}$ to $\mathrm{E}=0.4-1.0 \mathrm{MeV}$,' J. Phys. Chem. Ref. Data, vol. 24, no. 1, pp. 71-643, 1995.

[31] C. T. Chantler, "Detailed tabulation of atomic form factors, photoelectric absorption and scattering cross section, and mass attenuation coefficients in the vicinity of absorption edges in the soft $\mathrm{X}$-ray $(\mathrm{Z}=$ $30-36, \mathrm{Z}=60-89, \mathrm{E}=0.1 \mathrm{keV}-10 \mathrm{keV}$ ), addressing convergence issues of earlier work," J. Phys. Chem. Ref. Data, vol. 29, no. 4, pp. 597-1048, 2000.

[32] J. H. Hubbell, Photon Cross Sections, Attenuation Coefficients, and Energy Absorption Coefficients from $10 \mathrm{keV}$ to $100 \mathrm{GeV}$, National Bureau of Standards Washington, DC, USA: Rep. NSRDS-NBS 29, 1969.

[33] F. Fischer, "Beiträge zur theorie der absorption von röntgenstrahlung," Ann. Phys., vol. 400, no. 7, pp. 821-850, 1931

[34] F. Sauter, "Über den atomaren photoeffekt in der K-schale nach der relativistischen wellenmechanik Diracs," Ann. Phys., vol. 403, no. 4, pp. $454-488,1931$

[35] F. Sauter, "Über den atomaren photoeffekt bei großer härte der anregenden strahlung," Ann. Phys., vol. 401, no. 2, pp. 217-248, 1931.

[36] C. M. Davisson and R. D. Evans, "Gamma-ray absorption coefficients," Rev. Mod. Phys. 24, vol. 79, pp. 79-107, 1952.

[37] M. Gavrila, "Relativistic K-shell photoeffect," Phys. Rev., vol. 113, pp. 514-536, 1959 .

[38] B. Nagel, "Angular distribution and polarization of K-shell photoelectrons in the high energy limit," Ark. Fys., vol. 24, pp. 151-159, 1963.

[39] M. Gavrila, "Relativistic L-shell photoeffect," Phys. Rev., vol. 124, pp. 1132-1141, 1961

[40] W. H. McMaster, N. Kerr Del Grande, J. H. Mallet, and J. H. Hubbell, Compilation of X-ray cross sections, Section II Revision 1, Lawrence Livermore National Laboratory Rep. UCRL-50174, 1969.

[41] W. H. McMaster, N. Kerr Del Grande, J. H. Mallet and J. H. Hubbell, "Compilation of x-ray cross sections UCRL-50174, sections I, II revision 1, III, IV," Atom. Data Nucl. Data Tables, vol. 8, no. 4-6, pp. $443-444,1970$.

[42] A. Shaltout, H. Ebel, and R. Svagera, "Update of photoelectric absorption coefficients in the tables of McMaster," X-Ray Spectrom, vol. 35, pp. 52-56, 2006.

[43] E. Storm and H. I. Israel, "Photon cross sections from $1 \mathrm{keV}$ to $100 \mathrm{MeV}$ for elements $\mathrm{Z}=1$ to $\mathrm{Z}=100$," Atom. Data Nucl. Data Tables, vol. 7 , pp. $565-681,1970$

[44] H. Brysk and C. Zerby, "Photoelectric cross sections in the keV range," Phys. Rev., vol. 171, pp. 292-298, 1968.

[45] W. M. J. Veigele, "Photon cross sections from $0.1 \mathrm{keV}$ to $1 \mathrm{MeV}$ for elements Z $=1$ to Z = 94," Atom. Data Nucl. Data Tables, vol. 5, no. 1, pp. 51-111, 1973.

[46] E. B. Saloman, J. H. Hubbell, and J. H. Scofield, "X-Ray attenuation cross sections for energies $100 \mathrm{eV}$ to $100 \mathrm{keV}$ and elements $\mathrm{Z}=1$ to Z = 92," Atom. Data Nucl. Data Tables, vol. 38, pp. 1-197, 1988.

[47] E. B. Saloman and J. H. Hubbell, X-ray attenuation coefficients (total cross sections): Comparison of the experimental data base with the recommended values of Henke and the theoretical values of Scofield for energies between 0.1-100 keV, National Bureau of Standards Washington, DC, USA: Rep. NBSIR-86-3431, 1986.

[48] F. Biggs and R. Lighthill, Analytical Approximations For X-ray Crosssections, Sandia National Laboratories Albuquerque: Rep. SC-RR-66$452,1967$.

[49] F. Biggs and R. Lighthill, Analytical Approximations For X-ray Crosssections II, Sandia National Laboratories Albuquerque, NM, USA: Rep. SC-RR-710507, 1971.

[50] F. Biggs and R. Lighthill, Analytical Approximations for $X$ RayCrossSections III, Sandia National Laboratories, Albuquerque, NM, USA: Rep. SAND87-0070, 1988.

[51] B. L. Henke, P. Lee, T. Tanaka, R. L. Shimabukuro, and B. Fujikawa, "Low-energy X-ray interaction coefficients: Photoabsorption, scattering, and reflection: $\mathrm{E}=100-2000 \mathrm{eV} \mathrm{Z}=1-94$," Atom. Data Nucl. Data Tables, vol. 27, pp. 1-144, 1982.
[52] E. F. Plechaty, D. E. Cullen, and R. J. Howerton, Tables and graphs of photon-interaction cross sections from $0.1 \mathrm{keV}$ to $100 \mathrm{MeV}$ derived from the LLL evaluated-nuclear-Data library, Livermore National Laboratory UCRL-50400, Dec. 71978 vol. 6, Rev. 2

[53] V. M. Grishin, A. P. Kostin, S. K. Kotelnikov, and D. G. Streblechenko, "Parametrization of the photoabsorption cross section at low energies," Bull. Lebedev Inst., no. 6, pp. 1-6, 1994.

[54] B. L. Henke, E. M. Gullikson, and J. C. Davis, "X-ray interactions: Photoabsorption, scattering, transmission, and reflection at $\mathrm{E}=50-$ 30000 eV, Z = 1 - 92," Atom. Data Nucl. Data Tables, vol. 54, no. 2, pp. 181-342, 1993.

[55] D. K. Trubey, M. J. Berger, and J. H. Hubbell, Photon cross sections for ENDF/B-VI, Oak Ridge National Laboratory Rep. CONF-890408-4, 1989.

[56] PHOTX: Photon interaction cross section library Oak Ridge National Lab, RSICC Data Library DLC-136, 1989.

[57] C. L. Dunford, "Evaluated Nuclear Data File, ENDF/B-VI," Nuclear Data for Science and Technology, Berlin, Germany: Springer, pp. 788792, 1992.

[58] M. J. Berger et al., XCOM: Photon cross section database, Gaithersburg, MD: National Institute of Standards and Technology, 2010, http://physics.nist.gov/pml/data/xcom.

[59] M. Sanchez del Rio and R. J. Dejus, "XOP: A multiplatform graphical user interface for synchrotron radiation spectral and optics calculations," Proc. SPIE Materials, Manufacturing, and Measurement for Synchrotron Radiation Mirrors, vol. 3152, pp. 148-157, 1997.

[60] S. Brennan and P. L. Cowan, "A suite of programs for calculating xray absorption, reflection, and diffraction performance for a variety of materials at arbitrary wavelengths," Rev. Sci. Instrum., vol. 63, pp. 850-853, 1992.

[61] D. T. Cromer and D. A. Liberman, "Relativistic calculation of anomalous scattering factors for x-rays," J. Chem. Phys., vol. 53, pp. 1891$1898,1970$.

[62] D. Cullen et al., EPDL97, the Evaluated Photon Data Library, Lawrence Livermore National Laboratory Rep. UCRL-50400, 1997, vol. 6 , Rev. 5

[63] M. B. Chadwick et al., "ENDF/B-VII.1 nuclear data for science and technology: Cross sections, covariances, fission product yields and decay data," Nucl. Data Sheets, vol. 112, no. 12, pp. 2887-3152, 2011.

[64] J. H. Hubbell, H. A. Gimm, and I. Overbo, "Pair, triplet, and total cross sections (and mass attenuation coefficients) for $0.1 \mathrm{MeV}-100 \mathrm{GeV}$ photons in elements Z $=1$ to 100," J. Phys. Ref. Data, vol. 9, no. 4, pp. 1023-1147, 1980

[65] D. T. Cromer and D. A. Liberman, "Anomalous dispersion calculations near to and on the long-wavelength side of an absorption edge," Acta Cryst, vol. A37, pp. 267-268, 1981.

[66] L. Kissel, "RTAB: The rayleigh scattering database," Radiat. Phys. Chem., vol. 59, pp. 185-200, 2000.

[67] W. Elam, B. Ravel, and J. Sieber, "A new atomic database for X-ray spectroscopic calculations," Radiat. Phys. Chem., vol. 63, pp. 121-128, 2002.

[68] M. J. Berger and J. H. Hubbell, XCOM: Photon Cross Sections on a Personal Computer, National Bureau of Standards Gaithersburg, MD, USA: Rep. NBSIR 87-3597, 1987.

[69] E. F. Plechaty, D. E. Cullen, and R. J. Howerton, Tables and Graphs of Photon-Interaction Cross Sections From $0.1 \mathrm{keV}$ to $100 \mathrm{MeV}$ Derived from the LLL Evaluated-Nuclear-Data Library, Livermore National Laboratory UCRL-50400, 1981, vol. 6, Rev. 3

[70] G. P. Williams, "Electron binding energies of the elements," $C R C$ Handbook of Chemistry and Physics, 67th Boca Raton, FL, USA: CRC Press, 1986, pp. F163-166.

[71] H. Ebel, R. Svagera, M. F. Ebel, A. Shaltout, and J. H. Hubbell, "Numerical description of photoelectric absorption coefficients for fundamental parameter programs," X-Ray Spectrom, vol. 32, no. 6, pp. 442-451, 2003.

[72] W. R. Nelson, H. Hirayama, and D. W. O. Rogers, The EGS4 code system, Stanford, CA, USA: SLAC-265 Rep., 1985

[73] Y. Sakamoto, "Photon cross section data PHOTX for PEGS4 code," Proc. 3rd EGS4 Users Meeting, Japan: 1993, pp. 77-82.

[74] A. F. Bielajew and D. W. O. Rogers, Photoelectron angular distribution in the EGS4 code system, National Research Council of Canada Ottawa: ON Canada Rep. PIRS-0058, 1986.

[75] I. Kawrakow, E. Mainegra-Hing, D. W. O. Rogers, F. Tessier, and B. R. B. Walters, The EGSnrc Code System: Monte Carlo Simulation of Electron and Photon Transport, National Research Council of Canada Ottawa: ON Canada NRCC PIRS-701 Rep., 6th printing, 2013.

[76] S. M. Seltzer, "Electron-photon Monte Carlo calculations: The ETRAN code," Appl. Radiat. Isot., vol. 42, no. 10, pp. 917-941, 1991. 
[77] G. Battistoni et al., "The FLUKA code: Description and benchmarking," Proc. AIP Conf., 2007, vol. 896, pp. 31-49.

[78] A. Ferrari et al., Fluka: A multi-particle transport code, Geneva, Switzerland: Rep. CERN-2005-010, INFN/TC-05/11, SLAC-R-773, Oct. 2005.

[79] B. C. Franke, R. P. Kensek, and T. W. Laub, ITS5 theory manual, rev. 1.2, Sandia Natl. Lab. Albuquerque, NM, USA: Rep. SAND2004-4782, 2004

[80] X-5 Monte Carlo Team MCNP-A General Monte Carlo N-Particle Transport Code, Version 5, Los Alamos National Laboratory Rep. LA-UR-03-1987, 2003, revised 2008

[81] D. B. Pelowitz et al., MCNPX 2.7.E Extensions, Los Alamos National Laboratory Rep. LA-UR-11-01502, 2011.

[82] D. E. Cullen et al., Tables and Graphs of Photon Interaction Cross Sections from $10 \mathrm{eV}$ to $100 \mathrm{GeV}$ Derived from the $L L N L$ Evaluated Photon Data Library (EPDL), Lawrence Livermore National Laboratory, Rep. UCRL-50400, 1989, vol. 6, Rev. 4

[83] T. Goorley et al., Initial MCNP6 release overview - MCNP6 version 1.0, Los Alamos National Laboratory LA-UR-13-22934 Rep., 2013.

[84] D. Garber, ENDF/B Summary Documentation, BNL-17541, 1975

[85] J. Sempau, E. Acosta, J. Baro, J. M. Fernandez-Varea, and F. Salvat, "An algorithm for Monte Carlo simulation of coupled electron-photon transport," Nucl. Instrum. Methods Phys. Res. B, vol. 132, pp. 377-390, 1997.

[86] F. Salvat, J. M. Fernandez-Varea, and J. Sempau, "Penelope - a code system for Monte Carlo simulation of electron and photon transport," Proc. Workshop NEA 6416, 2008.

[87] F. Salvat, J. M. Fernandez-Varea, and J. Sempau, "Penelope-2011 a code system for Monte Carlo simulation of electron and photon transport," Proc. Workhop NEA/NSC/DOC(2011)5, 2011.

[88] "GEANT detector description and simulation tool," CERN Program Library Long Writeup W5013, 1995.

[89] Geant4 Collaboration"Geant4 physics reference manual," http:// geant4.web.cern.ch/geant4/UserDocumentation/UsersGuides/ PhysicsReferenceManual/fo/PhysicsReferenceManual.pdf

[90] J. Apostolakis, S. Giani, L. Urbanb, M. Maire, A. V. Bagulya, and V. M. Grichine, "An implementation of ionisation energy loss in very thin absorbers for the GEANT4 simulation package," Nucl. Instrum. Methods Phys. Res. A, vol. 453, no. 3, pp. 597-605, 2000.

[91] S. Chauvie, G. Depaola, V. Ivanchenko, F. Longo, P. Nieminen, and M. G. Pia, "Geant4 Low Energy Electromagnetic Physics," Proc. Computing in High Energy and Nuclear Physics, Beijing, China: 2001, pp. 337-340.

[92] S. Chauvie et al., "Geant4 low energy electromagnetic physics," Proc. IEEE Nucl. Sci. Symp. Conf. Rec., 2004, pp. 1881-1885.

[93] J. Apostolakis, S. Giani, M. Maire, P. Nieminen, M. G. Pia, and L. Urban, Geant 4 low energy electromagnetic models for electrons and photons, INFN/AE-99/18, 1999, Frascati.

[94] M. Batic, G. Hoff, M. G. Pia, and P. Saracco, "Photon elastic scattering simulation: Validation and improvements to geant4," IEEE Trans. Nucl. Sci., vol. 59, no. 4, pp. 1636-1664, Aug. 2012.

[95] A. Alexandrescu, Modern C++ Design, Boston, MA, USA: AddisonWesley, 2001

[96] S. Chauvie et al., "Geant4 physics processes for microdosimetry simulation: Design foundation and implementation of the first set of models," IEEE Trans. Nucl. Sci., vol. 54, no. 6, pp. 2619-2628, Dec. 2007.

[97] M. Augelli et al., "Research in geant4 electromagnetic physics design, and its effects on computational performance and quality assurance," Proc. IEEE Nucl. Sci. Symp. Conf. Rec., 2009, pp. 177-180.

[98] M. G. Pia et al., "Design and performance evaluations of generic programming techniques in a R\&D prototype of Geant4 physics," J. Phys.: Conf. Ser., vol. 219, p. 042019, 2010.

[99] G. Booch, J. Rumbaugh, and I. Jacobson, The Unified Modeling Language User Guide, Reading, MA, USA: Addison-Wesley, 1999.

[100] E. Gamma, R. Helm, R. Johnson, and J. Vlissides, Design Patterns, Reading, MA, USA: Addison-Wesley, 1995.

[101] M. Fowler, Refactoring: Improving the Design of Existing Code, Reading, MA, USA: Addison-Wesley, 1999.

[102] J. D. E. Beynon and R. B. Cairns, "An experimental determination of the photoabsorption cross section of atomic hydrogen," Proc. Phys. Soc., vol. 86, pp. 1343-1349, 1965

[103] J. L. Kohl, G. P. Lafyatis, H. P. Palenius, and W. H. Parkinson, "Absolute cross section for photoionization of atomic oxygen," Phys. Rev. A, vol. 18 , no. 2 , pp. $571-574,1978$

[104] H. P. Palenius, J. L. Kohl, and W. H. Parkinson, "Absolute measurement of the photoionization cross section of atomic hydrogen with a shock tube for the extreme ultraviolet," Phys. Rev. A, vol. 13, no. 5, pp. 18051816,1976
[105] B. L. Henke, R. L. Elgin, and R. B. Ledingham, "X-ray absorption in the 2 to 200 A region," Norelco Rep., vol. 14, pp. 112-116, 1967.

[106] J. F. Lowry, D. H. Tomboulian, and D. L. Ederer, "Photoionization cross section of helium in the 100 to $250 \AA$ A region," Phys. Rev., vol. 137, no. 4A, pp. 1054-1057, 1965.

[107] A. P. Lukirskii, I. A. Brytov, and T. M. Zimkina, "Photoionization absorption of $\mathrm{He}, \mathrm{Kr}, \mathrm{Xe}, \mathrm{CH} 4$, and methylal in the 23.6-250 ̊ region," Optics Spectrosc., vol. 17, pp. 234-238, 1964.

[108] G. V. Marr and J. B. West, "Aabsolute photoionization cross-section tables for helium, neon, argon, and krypton in the VUV spectral regions," Atom. Data Nucl. Data Tables, vol. 18, pp. 497-508, 1976.

[109] J. A. R. Samson, "Photoionisation cross sections of helium," J. Opt. Soc. Amer, vol. 54, no. 7, pp. 876-878, 1964.

[110] J. A. R. Samson and W. C. Stolte, "Precision measurements of the total photoionization cross-sections of $\mathrm{He}, \mathrm{Ne}, \mathrm{Ar}, \mathrm{Kr}$, and Xe," J. Electron Spectros. Relat. Phenomena, vol. 123, pp. 265-276, 2002.

[111] W. S. Watson, "Photoionization of helium, neon and argon in the 60$230 \mathrm{eV}$ photon energy range," J. Phys. B, vol. 5, no. 12, pp. 2292-2303, 1972.

[112] J. B. West and G. V. Marr, "The absolute photoionization cross sections of helium, neon, argon and krypton in the extreme vacuum ultraviolet region of the spectrum," Proc. Royal Soc. London A, vol. 349, no. 1658, pp. 397-421, 1976.

[113] D. J. Baker Jr. and D. H. Tomboulian, "Photoelectric K-absorption cross section of lithium," Phys. Rev., vol. 128, no. 2, pp. 677-680, 1962.

[114] M. T. Huang et al., "Single and double photoionization of lithium," Phys. Rev., vol. 59, no. 5, pp. 3397-3401, 1999

[115] G. Mehlman, J. W. Cooper, and E. B. Saloman, "Absolute photoabsorption cross section of the K shell of atomic lithium," Phys. Rev. A, vol. 25, no. 4, pp. 2113-2122, 1982

[116] T. J. Peterson Jr., E. J. McGuire, and D. H. Tomboulian, "Photoelectric K-absorption cross section of beryllium," Phys. Rev. A, vol. 129, no. 2, pp. 674-677, 1963.

[117] "Measurements of the ultrasoft X-ray absorption of Ar, Ne, $\mathrm{N}_{2}, \mathrm{O}_{2}, \mathrm{CH}_{4}$, He and $\mathrm{H}_{2}, " J$. Phys. D, vol. 3, pp. 1392-1398, 1970.

[118] J. A. R. Samson and G. C. Angel, "Single- and double- photoionization cross sections of atomic nitrogen from threshold to $31 \AA$," Phys. Rev. A, vol. 42 , no. 3 , pp. $1307-1312,1990$

[119] G. C. Angel and J. A. R. Samson, "Total photoionization cross sections of atomic oxygen from threshold to $44.3 \AA$," Phys. Rev. A, vol. 38 , no. 11 , pp. 5578-5585, 1988.

[120] R. B. Cairns and J. A. R. Samson, "Total absorption cross section of atomic oxygen below 910 A,," Phys. Rev., vol. 130, no. 5A, pp. 1403$1407,1965$.

[121] J. A. R. Samson and P. N. Pareek, "Absolute photoionization cross sections of atomic oxygen," Phys. Rev. A, vol. 31, no. 3, pp. 1470-1476, 1985.

[122] I. H. Suzuki and N. Saito, "Absolute photoabsorption cross-sections of $\mathrm{Ne}$ and $\mathrm{Xe}$ in the sub-keV X-ray region," J. Electr. Spectr. Related Phenomena, vol. 129, pp. 71-79, 2003.

[123] K. Codling, J. R. Hamley, and J. B. West, "The absolute photoabsorption cross section of atomic sodium in the region above the $2 p$ threshold (45$250 \mathrm{eV})$, , J. Phys. B, vol. 10, no. 14, pp. 2797-2807, 1977.

[124] R. Gowda and B. Sanjeevaiah, "K-shell photoelectric cross sections for 145-keV gamma rays," Phys. Rev. A, vol. 8, no. 5, pp. 2425-2428, 1973.

[125] J. A. R. Samson, Y. Shefer, and G. C. Angel, "A critical test of manybody theory: The photoionization cross section of $\mathrm{Cl}$ as an example of an open-shell atom," Phys. Rev. Lett., vol. 56, no. 19, pp. 2020-2023, 1986.

[126] A. P. Lukirskii and T. M. Zimkina, "Mass absorption coefficients of argon and ethyl alcohol in the ultrasoft X-ray region," Bull. Acad. Sci. USSR, Phys. Ser., vol. 27, pp. 808-811, 1963.

[127] I. H. Suzuki and N. Saito, "Total photoabsorption cross-section of Ar in the sub-keV energy region," Radiat. Phys. Chem., vol. 73, pp. 1-6, 2005 .

[128] M. Kato et al., "Absolute photoionization cross sections with ultrahigh energy resolution for $\mathrm{Ar}, \mathrm{Kr}, \mathrm{Xe}$ and $\mathrm{N}_{2}$ in inner-shell ionization region," J. Electr. Spectrosc. Rel. Phenom., vol. 160, pp. 39-48, 2007.

[129] B. X. Yang and J. Kirz, "Soft x-ray absorption cross section of argon determined by a variable absorber technique," App. Opt., vol. 26, no. 18, pp. 3823-3826, 1987.

[130] L. Zheng, M. Cui, Y. Zhao, J. Zhao, and K. Chen, "Total photoionization cross-sections of $\mathrm{Ar}$ and $\mathrm{Xe}$ in the energy range of 2.1-6.0 keV," $J$. Electr. Spectr. Related Phenomena, vol. 152, pp. 143-147, 2006.

[131] W. Sandner, T. F. Gallagher, K. A. Safinya, and F. Gounand, "Photoionization of potassium in the vicinity of the minimum in the cross section," Phys. Rev., vol. 23, no. 5, pp. 2732-2735, 1981. 
[132] O. SöC§üt, "Direct measurement of total atomic photoelectric, total atomic attenuation and total atomic scattering cross-sections in the atomic region $22 \leq \mathrm{Z} \leq 58$ at $59.5 \mathrm{keV}$ photons," J. Quant. Spectrosc. Ra., vol. 73, pp. 63-68, 2002.

[133] N. K. Del Grande, "Measured 1 to $40 \mathrm{keV}$ photoabsorption cross sections for: Fe, Ni, Sn, Ta, Pt, Au, Pb, U," Proc. SPIE 0691, X-Ray Imaging II, 1986, pp. 2-10.

[134] G. G. Lombardi, P. L. Smith, and W. H. Parkinson, "Measurement of the photoionization cross section of neutral iron," Phys. Rev., vol. 18, no. 5, pp. 2131-2134, 1978.

[135] R. Gowda and B. Sanjeevaiah, "Photoelectric cross sections for $279.1 \mathrm{keV}$ and $411.8 \mathrm{keV}$ rays," J. Phys. B: At. Mol. Phys, vol. 7, pp. $1772-1775,1974$.

[136] R. Gowda and B. Sanjeevaiah, "The total and k shell photoelectric cross sections for $661.6 \mathrm{keV}$ gamma rays," Can. J. Phys., vol. 53, no. 9, pp. 846-849, 1975

[137] C. Ranganathaiah, R. Gowda, and B. Sanjeevaiah, "Photoelectric cross sections for $323 \mathrm{keV}$ gamma rays in $\mathrm{Cu}, \mathrm{Zr}, \mathrm{Ag}, \mathrm{Sn}, \mathrm{Ta}, \mathrm{Au}$, and $\mathrm{Pb}$," J. Can. J. Phys., vol. 55, no. 18, pp. 1592-1593, 1977.

[138] W. F. Titus, "Total photoelectric cross sections of copper, molybdenum, silver, tantalum, and gold at $662 \mathrm{keV}, "$ Phys. Rev., vol. 115, no. 2, pp. 351-356, 1959.

[139] J. Lang and W. S. Watson, "The photoabsorption coefficients of krypton and xenon from $48 \AA$ to $210 \AA ̊$," J. Phys. B, vol. 8, no. 14, pp. 339-343, 1975

[140] I. H. Suzuki and N. Saito, "TPhotoabsorption cross-section for Kr in the sub-keV energy region,” J. Electr. Spectr. Related Phenomena, vol. 123, pp. 239-245, 2002.

[141] G. V. Marr and D. M. Creek, "The photoionization absorption continua for alkali metal vapours," Proc. Roy. Soc. A, vol. 304, pp. 233-244, 1968.

[142] A. M. Manna and V. V. Rao, "Photoelectric cross sections of 279 and $661 \mathrm{KeV}$ gamma rays in Y, Ce, Dy and Yb," J. Phys. B, At. Mol. Phys., vol. 18 , pp. 3161-3165, 1985.

[143] G. Budak, A. Karabulut, Ö. Şimşek, and M. Ertuĝrul, "Measurement of total atomic attenuation, total atomic photoelectric and total atomic scattering cross sections in the range $40 \leq \mathrm{Z} \leq 52$," Nucl. Instrum. Methods Phys. Res. B, vol. 149, pp. 379-382, 1999.

[144] H. A. Jahagirdar, B. Hanumaiah, and S. R. Thontadarya, "A new direct method to determine total atomic photoelectric cross sections at 123.6 keV," Appl. Radiat. Isot., vol. 43, no. 3, pp. 399-404, 1992.

[145] A. Lurio and W. Reuter, "Low energy photoionization cross sections from proton induced xray spectroscopy," Appl. Phys. Lett., vol. 27, pp. 704-706, 1975 .

[146] K. Parthasaradhi, V. Lakshminarayana, and S. Jnanananda, "Absolute photoelectric cross sections of 320-keV gamma rays in $\mathrm{Pb}, \mathrm{Sn}$, and $\mathrm{Ag}$," Phys. Rev., vol. 142, no. 1, pp. 9-11, 1966.

[147] N. Saito and I. H. Suzuki, "Precise photoabsorption cross sections of Ne and Xe," Nucl. Instrum. Methods Phys. Res. A, 467-468, pp. 1577-1580, 2001.

[148] T. B. Cook, F. B. Dunning, G. W. Folts, and R. F. Stebbings, "Absolute measurement of the photoionization cross section for ground-state cesium atoms," Phys. Rev. A, vol. 15, no. 4, pp. 1526-1529, 1977.

[149] M. Ertuĝrul, "Measurement of K, L and higher shell photoionization cross-sections at $59.5 \mathrm{keV}$," Anal. Chim. Acta., vol. 491, pp. 239-244, 2003.

[150] S. J. Anasuya, T. K. Umesh, and R. Gowda, "Photoeffect cross sections of some rare earth elements for $661.6 \mathrm{keV}$ gamma rays by a new method," Radiat. Phys. Chem., vol. 51, no. 4-6 pp. 363-364, 1998.

[151] H. A. Jahagirdar, B. Hanumaiah, and S. R. Thontadarya, "Measurement of total and photoelectric cross sections of rare earth elements present in compounds for $123.6 \mathrm{keV}$ and $145.4 \mathrm{keV}$ photons," Radiat. Phys. Chem., vol. 47, no. 6, pp. 801-805, 1996.

[152] A. Karabulut, G. Budak, and M. Ertugrul, "Fast measurement of total atomic attenuation, total atomic photoelectric and total atomic scattering cross sections in the range $58 \leq \mathrm{Z} \leq 68$ using radioisotope $\mathrm{X}$-ray fluorescence," Nucl. Instrum. Methods Phys. Res. B, vol. 152, no. 2, pp. 202-206, 1999

[153] M. Ertuĝrul, Ö. Şimşek, O. Doĝan, and Ü. Turgut, "Direct determination of total atomic attenuation, total atomic photoelectric and total atomic scattering cross sections of Gd, Tb, Dy and Er at $60 \mathrm{keV}$," J. Radioanal. Nucl. Chem., Lett., vol. 213, no. 1, pp. 37-44, 1996.

[154] S. V. Nayak and N. M. Badiger, "A novel method for measuring Kshell photoelectric parameters of high-Z elements," J. Phys. B, vol. 39, pp. 2893-2900, 2006.

[155] A. M. Ghose, "Photoelectric cross sections of gamma rays for heavy elements," Nucl. Phys., vol. 75, pp. 539-548, 1966.
[156] S. J. Anasuya, T. K. Umesh, and R. Gowda, "A new method to determine the atomic photo effect cross section in lead at $661.6 \mathrm{keV}$," Nucl. Instrum. Methods Phys. Res. B, vol. 84, pp. 421-424, 1994.

[157] J. M. Bizau, P. Gerard, and F. J. Wuilleumier, "Photoionization of atomic Ca in the 26-120-eV photon-energy range," Phys. Rev. A, vol. 36, no. 3, pp. 1220-1248, 1987.

[158] K. Codling, R. G. Houlgate, J. B. West, and P. R. Woodruff, "Angular distribution and photoionization measurements on the $2 \mathrm{p}$ and $2 \mathrm{~s}$ electrons in neon," J. Phys. B, vol. 9, no. 5, pp. L83-L86, 1976.

[159] M. J. Lynch, A. B. Gardner, K. Godling, and G. V. Marr, "The photoionization of the $3 \mathrm{~s}$ subshell of argon in the threshold region by photoelectron spectroscopy," Phys. Lett., vol. 43A, no. 3, pp. 237-238, 1973.

[160] R. G. Houlgate, J. B. West, K. Codling, and G. V. Marr, "Angular distribution and photoionization cross section measurements on the $3 p$ and 3s subshells of argon," J. Phys. B, vol. 7, no. 17, pp. L470-L473, 1974.

[161] B. Möbus, B. Magel, K. H. Schartner, B. Langer, U. Becker, M. Wildberger, and H. Schmoranzer, "Measurements of absolute Ar 3s photoionization cross sections," Phys. Rev. A, vol. 47, no. 5, pp. 38883893, 1993.

[162] J. A. R. Samson and J. L. Gardner, "Photoionization cross sections of the outer s-subshell electrons in the rare gases," Phys. Rev. Lett., vol. 33, no. 12 , pp. 671-673, 1974.

[163] J. Jiménez-Mier, M. O. Krause, and P. Gerard, "Partial and total cross sections and multiplet structure in the photoionization of atomic manganese," Phys. Rev. A, vol. 40, no. 7, pp. 3712-3720, 1989.

[164] S. K. Arora, K. L. Allawadhi, and B. S. Sood, "K-shell photoelectric cross-section measurement," Phys. Rev. A, vol. 23, no. 3, pp. 1147-1151, 1981.

[165] R. Gowda and B. Sanjeevaiah, "K-shell photoelectric cross sections of $\mathrm{Cu}, \mathrm{Zr}, \mathrm{Ag}, \mathrm{Sn}, \mathrm{Ta}, \mathrm{Au}$, and $\mathrm{Pb}$ for 279.1- and $411.8-\mathrm{keV} \gamma$ rays," Phys. Rev. A, vol. 10, no. 4, pp. 1188-1191, 1974.

[166] A. Karabulut, A. Gürol, G. Budak, and M. Ertugrul, "K shell and L subshell and $\mathrm{L}$ shell photoeffect cross-sections in the atomic region $40<$ $\mathrm{Z} \leq 52$ and $58 \leq \mathrm{Z} \leq 68$ at $59.537 \mathrm{keV}$," Nucl. Instrum. Methods Phys. Res. B, vol. 227, pp. 485-489, 2005.

[167] C. Ranganathaiah, R. Gowda, and B. Sanjeevaiah, "K-shell photoionization cross sections for $514,661.6,765.8$, and $1115.5 \mathrm{keV}$ gamma rays," J. Phys. B, vol. 12, no. 12, pp. 1965-1971, 1979.

[168] C. Ranganath, R. Gowda, and B. Sanjeevaiah, "A coincidence method of measuring K-shell photoelectric cross sections," Nucl. Instrum. Methods, vol. 154, pp. 331-334, 1978.

[169] C. Ranganathaiah, R. Gowda, and B. Sanjeevaiah, "Atomic K-shell photoionization cross sections for Sc 46 and Co $60 \gamma$ rays," Phys. Rev. A, vol. 23, no. 4, pp. 1841-1847, 1981.

[170] T. Gustafsson, "Photoionization cross sections of the 5s electrons in Xe measured with synchrotron radiation," Chem. Phys. Lett., vol. 51, no. 2, pp. 383-385, 1977.

[171] U. Becker, H. G. Kerkhoff, M. Kupsch, B. Langer, D. Szostak, and R. Wehlitz, "Photoionization of xenon with soft X-rays," J. Phys. Colloq., vol. 48, pp. 497-500, 1987.

[172] J. M. Bizau, D. Cubaynes, P. Gerard, and F. J. Wuilleumier, "Photoionization of atomic barium: Ba atoms in the ground state," Phys. Rev. A, vol. 40, no. 6, pp. 3002-3025, 1989.

[173] S. V. Nayak and N. M. Badiger, "Measurement of K shell photoelectric cross sections at a K edge-a laboratory experiment," Eur. J. Phys., vol. 28, pp. 859-866, 2007.

[174] S. B. Hosur, L. R. Naik, and N. M. Badiger, "Study of the K shell photoelectric parameters of $\mathrm{Dy}, \mathrm{Yb}$ and $\mathrm{W}$ atoms using low energy bremsstrahlung radiation," Eur. Phys. J. D, vol. 62, pp. 155-161, 2011.

[175] S. V. Nayak and N. M. Badiger, "Measurement of K-shell photoelectric absorption parameters of $\mathrm{Hf}, \mathrm{Ta}, \mathrm{Au}$, and $\mathrm{Pb}$ by an alternative method using a weak $\beta$ source," Phys. Rev. A, vol. 73, no. 3, p. 032707 , 2006.

[176] K. L. Allawadhi, S. K. Arora, and B. S. Sood, "Measurement of L-shell photoelectric cross sections in high $\mathrm{Z}$ elements at $60 \mathrm{keV}$," Pramana, vol. 10 , no. 5, pp. 511-517, 1978 .

[177] C. Kunz, S. Thiess, B. Cowie, T.-L. Lee, and J. Zegenhagen, “Outer subshell photoabsorption cross-sections determined by X-ray photoelectron spectroscopy up to $14.5 \mathrm{keV}, "$ Nucl. Instrum. Methods Phys. Res. A, vol. 547, pp. 73-86, 2005.

[178] K. L. Allawadhi, B. S. Ghumman, and B. S. Sood, "Measurement of Lshell photoelectric cross sections in high Z elements at 37 and $74 \mathrm{keV}$," Pramana, vol. 8, no. 5, pp. 433-437, 1977.

[179] S. K. Arora, K. L. Allawadhi, and B. S. Sood, "Measurement of L III subshell photoelectric cross-sections in high Z-elements," Pramana, vol. 16, no. 6, pp. 467-472, 1981 . 
[180] E. J. Bleeker, P. F. A. Goudsmit, and C. De Vries, "Measurements of k shell photoelectric cross sections," Nucl. Phys., vol. 29, pp. 452-461, 1962.

[181] H. W. Boyd, W. H. Brantley, and J. H. Hamilton, "Photoelectric crosssections for the $\mathrm{L}, \mathrm{M}$ and $\mathrm{N}$ shells of uranium for photons of $122 \mathrm{keV}$," Nuovo Cimento, vol. 39, no. 1, pp. 1-9, 1965.

[182] K. S. Babu, S. C. Lingam, and D. V. K. Reddy, "Total mass attenuation cross sections of rare earth elements in the energy range 30 to $662 \mathrm{keV}$ and derived photoelectric cross sections," Physica C, vol. 125, pp. 353360, 1984.

[183] A. J. Bearden, "X-ray photoeffect cross sections in low and mediumZ absorbers for the energy range $852 \mathrm{eV}$ to $40 \mathrm{keV}$," J. Appl. Phys., vol. 37, no. 4, pp. 1681-1692, 1966.

[184] K. P. Chand, D. K. S. Reddy, V. R. K. Murty, J. R. Rao, and V. Lakshminarayana, "Photoelectric cross sections of low-energy photons in light elements," J. Phys. B, vol. 9, no. 2, pp. 177-184, 1976.

[185] B. E. Cole, "Empirical photoabsorption cross sections for C, N, O, F and $\mathrm{Cl}$ obtained from molecular measurements between 50 and $340 \AA$," J. Quant. Spectrosc. Radiat. Transfer, vol. 19, pp. 467-471, 1978

[186] S. A. Colgate, "Gamma-ray absorption measurements," Phys. Rev., vol. 87 , no. 4, pp. 592-601, 1952

[187] W. Dachun et al., "X-ray attenuation coefficients and photoelectric cross sections of $\mathrm{Cu}$ and $\mathrm{Fe}$ in the range $3 \mathrm{keV}$ to $29 \mathrm{keV}$," Nucl. Instrum. Methods Phys. Res. B, vol. 71, pp. 241-248, 1992.

[188] W. Dachun, Y. Hua, L. Pingan, and D. Xunliang, "Measurements of mass attenuation coefficients of yttrium with characteristic lines from elements excited by energetic protons," Nucl. Instrum. Methods Phys. Res. B, vol. 71, pp. 249-254, 1992.

[189] L. Gerward, "X-ray attenuation coefficients and atomic photoelectric absorption cross sections of silicon," J. Phys. B, vol. 14, pp. 3389-3395, 1981.

[190] L. Gerward, "Atomic photoeffect cross sections for beryllium, carbon, silicon and copper from 5 to $20 \mathrm{keV}$," J. Phys. B, vol. 22, pp. 1963-1969, 1989.

[191] R. Gowda and B. Sanjeevaiah, "Photoelectric cross sections for 72.1$\mathrm{keV} x$ rays in $\mathrm{Al}, \mathrm{Cu}, \mathrm{Zr}, \mathrm{Ag}, \mathrm{Sn}, \mathrm{Ta}, \mathrm{Au}$, and $\mathrm{Pb}$ derived from a total attenuation-coefficient measurement," Phys. Rev. A, vol. 9, no. 6, pp. $2569-2572,1974$

[192] S. C. Lingam, K. S. Babu, and D. V. Krishna Reddy, "Photoelectric cross sections around the K edge," Physica C, vol. 122, pp. 348-352, 1983.

[193] F. Machali, G. G. Al-Barakati, A. A. El-Sayed, and W. J. Altaf, "The photoelectric cross section of gamma rays in the energy range 43 to 152 keV," J. Phys. F, vol. 17, pp. 1279-1284, 1987.

[194] R. H. Millar, "Atomic number dependence of the photoelectric cross section for photons in the energy range from 4.5 to $25 \mathrm{keV}$," J. Phys. B, vol. 8, no. 12, pp. 2015-2022, 1975.

[195] V. R. K. Murty, K. S. Rao, K. Parthasaradhi, J. R. Rao, and V. Lakshminarayana, "Photoelectric interaction around the K edge," $J$. Phys. B, vol. 10, pp. 3189-3194, 1977.

[196] V. R. K. Murty, D. P. Winkoun, and K. R. S. Devan, "Total photoelectric cross sections close to absorption edges," Radiat. Phys. Chem., vol. 51, no. 4-6, pp. 361-362, 1998.

[197] R. Nathuram, I. S. S. Rao, and M. K. Mehta, "Photoelectric cross sections for 6-20 keV photons in beryllium, carbon, magnesium, aluminum, silicon, copper, silver, and lead," Phys. Rev. A, vol. 13, no. 12, pp. 4978-4981, 1988 .

[198] K. Parthasaradhi, "Photoelectric cross sections of gamma rays in $\mathrm{Al}, \mathrm{Cu}$, $\mathrm{Sn}, \mathrm{W}, \mathrm{Au}$ and $\mathrm{Pb}$ in the energy region 50-208 keV," J. Phys. A, vol. 1, pp. 256-258, 1968.

[199] K. Parthasaradhi and H. H. Hansen, "Attenuation-coefficient measurements for 3.3- to $165.8-\mathrm{keV}$ photons: Analysis in terms of total photoelectric cross sections," Phys. Rev. A, vol. 10, no. 2, pp. 563-568, 1974.

[200] K. Parthasaradhi, "Atomic photoelectric effect near threshold edges," Phys. Rev. A, vol. 32, no. 1, pp. 194-198, 1985.

[201] K. Parthasaradhi, "Photon cross sections near the x-ray-absorption edges of Ti, Ni, Pt, and Au," Phys. Rev. A, vol. 38, no. 3, pp. 1608-1611, 1988.

[202] P. P. Pawar and G. K. Bichile, "Photoelectric cross sections deduced from the measured total photon interaction cross sections for five elements $(24<\mathrm{Z}<)$ at $279.30 \mathrm{keV}$," J. Chem. Pharm. Res., vol. 3, pp. $212-217,2011$.

[203] R. Prasad, "Total photon-absorption cross-section measurements at 52.4, 60, 72.2, and 84.4 keV in Al, Fe, Mo, Ag, W, and Pt: Photoelectric cross sections deduced," Phys. Rev. A, vol. 18, no. 5, pp. 2167-2169, 1978.

[204] R. Prasad, "Photoelectric cross-sections for Cd and In at 22.2, 59.6 and $88.8 \mathrm{keV}$," Nucl. Instrum. Methods Phys. Res. A, vol. 255, pp. 52-53, 1987
[205] P. V. R. Rao, V. V. Rao, J. R. Rao, and K. Parthasaradhi, "Photoelectric cross-sections of gamma-rays," Lett. Nuovo Cimento, vol. 1, no. 6, pp. 311-312, 1969.

[206] K. S. Rao, P. V. R. Rao, B. V. T. Rao, and K. Parthasaradhi, "Photoelectric effect of gamma-rays," Lett. Nuovo Cimento, vol. 8, no. 6, pp. 373-377, 1973.

[207] K. Sivasankara Rao, V. Radha Krishna Murty, K. Parthasaradhi, J. Rama Rao, and V. Lakshminarayana, "Total to K-shell photoelectric cross section ratios in $\mathrm{Zr}, \mathrm{Ag}$, Ta, and Th," Pramana, vol. 9, no. 4, pp. 321328, 1977.

[208] V. Visweswara Rao Shahnawaz, "Photoelectric cross-sections of lowenergy photons," Nuovo Cimento, vol. 44A, no. 2, pp. 181-186, 1978.

[209] V. Visweswara Rao Shahnawaz and D. Venkateswara Rao, "Photoelectric cross sections for X-ray photons in low- and midium-Z elements," Physica C, vol. 111, pp. 107-110, 1981.

[210] D. Venkateswara Rao and V. Visweswara Rao, "Photoelectric crosssections at K and L edges," Nuovo Cimento, 67Ano. 2, pp. 143-150, 1982.

[211] A. S. Nagaswara Rao, A. Perumallu, and G. Krishna Rao, "Photon cross section measurements in compounds and elements in the energy range 30-660 keV," Physica C, vol. 124, pp. 96-104, 1984.

[212] D. K. S. Reddy, K. Premachand, and V. Radha Krishna Murty, "Photoelectric interaction below the K edge," Phys. Rev. A, vol. 13, no. 1, pp. 326-329, 1976.

[213] D. V. Krishna Reddy, S. Chandralingam, and K. Suresh Babu, "Photoelectric cross sections in heavy elements derived from total attenuation measurements," Physica C, vol. 132, pp. 403-406, 1985.

[214] D. Dattatreya Reddy et al., "Photoelectric effect aroundK-edges in the elements Ce, Sm, Dy and Yb," Lett. Nuovo Cimento, vol. 44, no. 6, pp. 398-400, 1985.

[215] B. Seetharami Reddy, K. Premachand, P. V. Ramana Rao, and K. Parthasaradhi, "Z-dependence of photoelectric cross-section in the energy region of absorption edges (6.4-136.47) keV," Nuovo Cimento, vol. 105, no. 5, pp. 735-379, 1992.

[216] B. Roy, B. K. Chatterjee, S. C. Roy, N. Bhattacharya, and N. Choudhury, "Photoelectric cross-sections derived from measured total attenuation coefficient of photons near absorption edges of heavier atoms," Appl. Radiat. Isot., vol. 48, no. 6, pp. 785-788, 1997.

[217] K. S. R. Sarma, K. L. Narasimham, K. Premchand, S. B. Reddy, K. Parthasaradhi, and V. Lakshminarayana, "Photoelectric crosssections of light elements and compounds at low photon energies," Pramana, vol. 18, no. 6, pp. 485-494, 1982.

[218] V. V. Shanawaz Rao, "Low-energy photon attenuation coefficients," Curr. Sci., vol. 46, no. 8, pp. 256-256, 1977.

[219] B. Sinha and N. Chaudhuri, "Experimental photoionization cross sections of gamma ray photons for low, medium and high $\mathrm{Z}$ atoms," Proc. Int. Symp. Radiation Physics, Calcutta, India: Nov. 4, 1974, pp. 20-22.

[220] F. Titus, "The photoelectric cross sections of Sn, Ta and Au at $2.62 \mathrm{MeV}, "$ Nucl. Phys., vol. 69, pp. 179-185, 1965.

[221] T. K. Umesh and C. Ranganathaiah, "A simple method of determining the photoeffect cross sections of elements for gamma rays," Nucl. Instrum. Methods Phys. Res. B, vol. 5, pp. 472-475, 1984.

[222] T. K. Umesh, C. Ranganathaiah, and B. Sanjeevaiah, "Photoeffect cross sections of some rare-earth elements at $145.4 \mathrm{keV}$," Phys. Rev. A, vol. 32, no. 2, pp. 959-962, 1985 .

[223] T. Sandiago, K. Umesh, and R. Gowda, "Photon interaction crosssections and anomalous scattering factors of $\mathrm{Cu}$ and Ag," Pramana, vol. 48, no. 5, pp. 1077-1090, 1997.

[224] K. L. Allawadhi and B. S. Sood, "K-shell photoelectric cross section for intermediate $\mathrm{Z}$ elements at 32 and $24 \mathrm{keV}$," Phys. Rev. A, vol. 11 no. 6, pp. 1928-1932, 1975.

[225] K. L. Allawadhi, S. L. Verma, B. S. Ghumman, and B. S. Sood, "Measurement of $\mathrm{K}$ shell photoelectric cross section of Ba K X-rays in Y, Zr, Mo, Ag, Cd and Sn," Physica C, vol. 95, pp. 424-431, 1978

[226] S. K. Arora, K. L. Allawadhi, and B. S. Sood, "L-shell photoelectric cross section measurements," J. Phys. B, vol. 14, pp. 1423-1432, 1981.

[227] S. K. Arora, K. L. Allawadhi, and B. S. Sood, "Relative L-shell photoelectric cross-section measurements in $\mathrm{W}, \mathrm{Pb}$ and $\mathrm{U}, "$ Physica $C$ vol. 106, pp. 155-160, 1981.

[228] M. Ertugrul et al., "Measurement of $\mathrm{L}_{i}$ subshell photoionization crosssections of W, Au and Bi at $31.6 \mathrm{keV}$," Radiat. Phys. Chem., vol. 65 , pp. 123-126, 2002.

[229] A. Gürol, A. Karabulut, R. Polat, G. Budak, Y. Şahin, and M. Ertugrul, "L-subshell and total L-shell photoeffect cross-sections measurements for $\mathrm{Pb}, \mathrm{Au}, \mathrm{W}$, and $\mathrm{Ta}$ at $59.5 \mathrm{keV}$," Radiat. Phys. Chem., vol. 66, pp. 197-205, 2003. 
[230] A. Karabulut, A. Gürol, G. Budak, and R. Polat, "Measurements of L subshell and total L shell photoeffect cross-sections for some elements in $72 \leq \mathrm{Z} \leq 92$," Eur. Phys. J. D, vol. 21, pp. 57-61, 2002.

[231] N. Kaya, G. Apaydn, V. Aylkc, E. Cengiz, and E. Traşoğlu, "K shell, $\mathrm{L}$ shell-subshell and $\mathrm{M}$ shell-subshell photoeffect cross-sections in elements between $\mathrm{Tb}(\mathrm{Z}=65)$ and $\mathrm{U}(\mathrm{Z}=92)$ at $123.6 \mathrm{keV}$, , Radiat. Phys. Chem., vol. 77, no. 2, pp. 101-106, 2008.

[232] R. C. G. Leckey, "Subshell photoionization cross sections of the elements for Al K $\alpha$ radiation," Phys. Rev. A, vol. 13, no. 3, pp. 1043-1051, 1976.

[233] M. L. Mallikarjuna, S. B. Appaji Gowda, K. E. Ganesh, R. Gowda, and T. K. Umesh, "A method to determine the K-shell photoeffect cross sections of some rare earth elements," Nucl. Instrum. Methods Phys. Res. $B$, vol. 215, pp. 4-8, 2004.

[234] K. Parthasaradhi, "Note on total to subshell photoelectric cross sections ratios," Nucl. Instrum. Methods, vol. 85, pp. 147-150, 1970.

[235] K. Parthasaradhi, "Note on the methods of determining the subshell photoelectric cross sections," Nucl. Instrum. Methods, vol. 81, pp. 205-206, 1970.

[236] K. Parthasaradhi et al., "Photon cross sections near the X-ray-absorption edges of Ti, Ni, Pt, and Au," Phys. Rev. A, vol. 38, no. 3, pp. 1608-1611, 1988.

[237] R. S. Prakhya, K. Parthasaradhi, V. Lakshminarayana, K L. Narasimham, K. V. Ramanaiah, and S. Bhuloka Reddy, "Measurement of K-shell photoelectric cross sections by the indirect method," Phys. Rev. A, vol. 33, no. 4, pp. 2440-2443, 1986.

[238] K. Sivasankara Rao, B. V. Tirumala Rao, B. Mallikarjuna Rao, and K. Parthsaradhi, "Shell-wise photoelectric cross-sections of gammarays," Nuovo Cimento, vol. 13, no. 1, pp. 267-270, 1973.

[239] K. S. Rao et al., "Photoelectric effect of gamma rays," Lett. Nuovo Cimento, vol. 8, no. 6, pp. 373-377, 1973.

[240] G. K. Rao, A. Perumallu, and A. S. N. Rao, "A study on atomic photo effect cross sections at the K-edge in high Z-elements," Physica, 125C, pp. 334-340, 1984

[241] D. V. Rao, "Measurements of photoelectric cross sections for X-ray and $\gamma$-ray photons at $\mathrm{K}, \mathrm{L}_{1}$ and $\mathrm{L}_{3}$ edges in $\mathrm{Ce}, \mathrm{Gd}$, $\mathrm{Dy}, \mathrm{Er}, \mathrm{Yb}$ and $\mathrm{Pb}$ : $\mathrm{An}$ analysis of the derived shell ratios," Nucl. Instrum. Methods Phys. Res. A, vol. 280, no. 2, pp. 222-225, 1989.

[242] D. D. Reddy et al., "Photoelectric Effect Around K-Edges in the Elements Ce, Sm, Dy and Yb," Lett. Nuovo Cimento, vol. 44, no. 6 , pp. 398-400, 1985.

[243] P. R. Sasi, V. Lakshimi Narayana, K. Parthasardhi, K. L. Narasimhan, S. Bhuloka Reddy, and K. V. Ramaniah, "Measurement of K-shell photoelectric cross sections using a direct method," Nucl. Instrum. Methods Phys. Res. A, vol. 256, pp. 373-376, 1987.

[244] M. I. Singh and B. S. Sood, "L-Shell photoelectric cross-sections for heavy atoms at 145 and $279 \mathrm{keV}$," Nuovo Cimento, vol. 8B, no. 1, pp. 261-270, 1972 .

[245] N. Singh, R. Mittal, K. L. Allawadhi, and B. S. Sood, "Measurement of L-shell photoelectric cross-sections in lower intermediate $\mathrm{Z}$ elements at 6 keV," Pramana, vol. 22, no. 2, pp. 89-92, 1984.

[246] J. A. Huwaldt, PlotDigitizer http://plotdigitizer.sourceforge.net/

[247] A. Wald and J. Wolfowitz, "An exact test for randomness in the nonparametric case, based on serial correlation," Ann. Math. Stat., vol. 14, pp. 378-388, 1943.
[248] IEEE standard for software verification and validation, IEEE Std 10122012 May 2012, IEEE Computer Society.

[249] ISO/IEC "International standard, information technology software life cycle process, IS0 12207," 2008-2011

[250] G. A. P. Cirrone et al., "A goodness-of-fit statistical toolkit," IEEE Trans. Nucl. Sci., vol. 51, no. 5, pp. 2056-2063, Oct. 2004.

[251] B. Mascialino, A. Pfeiffer, M. G. Pia, A. Ribon, and P. Viarengo, "New developments of the goodness-of-fit statistical toolkit," IEEE Trans. Nucl. Sci., vol. 53, no. 6, pp. 3834-3841, Dec. 2006.

[252] R. Core Team, "R: A language and environment for statistical computing," R Foundation for Statistical Computing, Vienna, Austria: 2012, ISBN 3-900051-07-0 http://www.R-project.org/

[253] R. K. Bock and W. Krischer, The Data Analysis BriefBook, Berlin, Germany: Springer, 1998

[254] A. G. Frodesen, O. Skjeggestad, and H. Tøfte, "Probability and statistics in particle physics," Universitetsforlaget, Bergen, 1979.

[255] M. Paterno, Calculating Efficiencies and Their Uncertainties, Fermi National Accelerator Batavia, IL, USA: Rep. FERMILAB-TM-2286CD, 2004.

[256] K. Pearson, "On the $\chi^{2}$ test of goodness of fit," Biometrika, vol. 14, no. 1-2, pp. 186-191, 1922.

[257] R. A. Fisher, "On the interpretation of $\chi^{2}$ from contingency tables, and the calculation of P," J. Royal Stat. Soc., vol. 85, no. 1, pp. 87-94, 1922

[258] R. D. Boschloo, "Raised conditional level of significance for the $2 \times 2$ table when testing the equality of two probabilities," Stat. Neerlandica, vol. 24, pp. 1-35, 1970 .

[259] S. Suissa and J. J. Shuster, "Exact unconditional sample sizes for the $2 \times 2$ binomial trial," J. Royal Stati. Soc., Ser. A, vol. 148, pp. 317-327, 1985.

[260] T. J. Santner and M. K. Snell, "Small-sample confidence intervals for $\mathrm{p}_{1}-\mathrm{p}_{2}$ and $\mathrm{p}_{1} / \mathrm{p}_{2}$ in $2 \times 2$ contingency tables," J. Amer. Stat. Ass., vol. 75 , no. 370, pp. 386-394, 1980.

[261] G. A. Barnard, "Significance tests for 22 tables," Biometrika, vol. 34, pp. 123-138, 1947.

[262] E. B. Wilson, "Barnard's CSM test of significance," Proc. National Acad. Sci. USA, 1952, vol. 38, no. 10, pp. 899-905.

[263] S. Mato and M. Andres, "Simplifying the calculation of the P-value for barnards test and its derivatives," Stat. Comp., vol. 7, pp. 137-143, 1997.

[264] A. Agresti, "A survey of exact inference for contingency tables," Stat. Sci., vol. 7, pp. 131-153, 1992.

[265] A. Martin Andres and A. Silva Mato, "Choosing the optimal unconditioned test for comparing two independent proportions," Comp. Stat. Data Anal., vol. 17, pp. 555-574, 1994.

[266] A. Martin Andres, A. Silva Mato, J. M. Tapia Garcia, and M. J. Sanchez Quevedo, "Comparing the asymptotic power of exact tests in $2 \tilde{\mathrm{A}}-2$ tables," Comp. Stat. Data Anal., vol. 47, pp. 745-756, 2004.

[267] S. T. Perkins et al., Tables and Graphs of Atomic Subshell and Relaxation Data Derived from the LLNL Evaluated Atomic Data Library (EADL), Z=1-100 UCRL-50400, 1997, vol. 30 . 